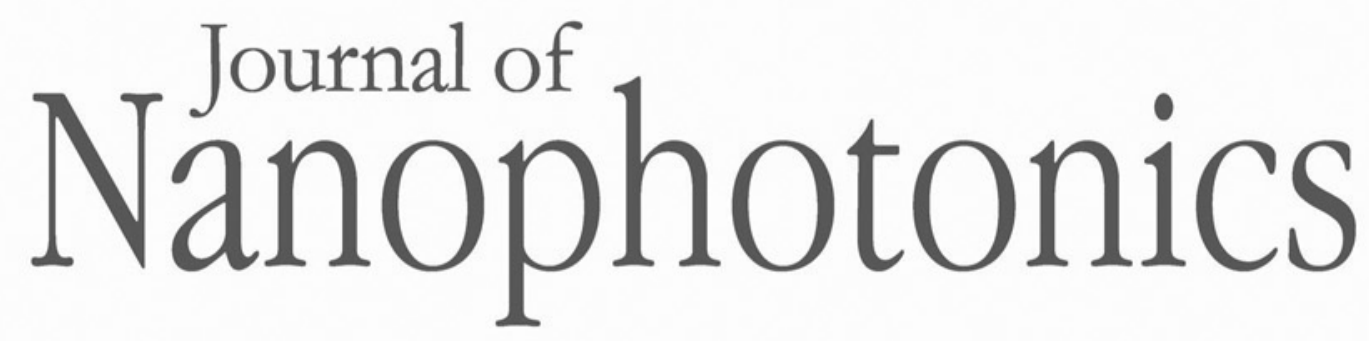

\title{
Review of nanophotonics approaches using nanostructures and nanofabrication for III-nitrides ultraviolet-photonic devices
}

\author{
Mohd Sharizal Alias \\ Malleswararao Tangi \\ Jorge A. Holguin-Lerma \\ Edgars Stegenburgs \\ Abdullah A. Alatawi \\ Islam Ashry \\ Ram Chandra Subedi \\ Davide Priante \\ Mohammad Khaled Shakfa \\ Tien Khee Ng \\ Boon S. Ooi
}

Mohd Sharizal Alias, Malleswararao Tangi, Jorge A. Holguin-Lerma, Edgars Stegenburgs, Abdullah A. Alatawi, Islam Ashry, Ram Chandra Subedi, Davide Priante, Mohammad Khaled Shakfa, Tien Khee Ng, Boon S. Ooi, "Review of nanophotonics approaches using nanostructures and nanofabrication for III-nitrides ultraviolet-photonic devices," J. Nanophoton. 12(4), 043508 (2018),

doi: $10.1117 / 1 . J N P .12 .043508$. 


\title{
Review of nanophotonics approaches using nanostructures and nanofabrication for III-nitrides ultraviolet-photonic devices
}

\author{
Mohd Sharizal Alias,* Malleswararao Tangi, \\ Jorge A. Holguin-Lerma, Edgars Stegenburgs, Abdullah A. Alatawi, \\ Islam Ashry, Ram Chandra Subedi, Davide Priante, \\ Mohammad Khaled Shakfa, Tien Khee Ng, and Boon S. Ooi* \\ King Abdullah University of Science and Technology (KAUST), Photonics Laboratory, \\ Thuwal, Saudi Arabia
}

\begin{abstract}
Group III-nitride semiconductor materials especially AlGaN are key-emerging candidates for the advancement of ultraviolet (UV) photonic devices. Numerous nanophotonics approaches using nanostructures (e.g., nanowires, nanorods, and quantum dots/disks) and nanofabrication (e.g., substrate patterning, photonic crystals, nanogratings, and surface-plasmons) have been demonstrated to address the material growth challenges and to enhance the device efficiencies of photonic devices operating at UV wavelengths. Here, we review the progress of nanophotonics implementations using nanostructured interfaces and nanofabrication approaches for the group III-nitride semiconductors to realize efficient UV-based photonic devices. The existing challenges of nanophotonics applications are presented. This review aims to provide analysis of state-of-the-art nanophotonic approaches in advancing the UV-photonic devices based on group III-nitride semiconductors. (C) The Authors. Published by SPIE under a Creative Commons Attribution 3.0 Unported License. Distribution or reproduction of this work in whole or in part requires full attribution of the original publication, including its DOI. [DOI: 10.1117/1.JNP.12 .043508]
\end{abstract}

Keywords: UV semiconductors; III-nitrides; photonic devices; nanostructures; nanophotonics.

Paper 18047SSV received Apr. 15, 2018; accepted for publication Jun. 4, 2018; published online Jul. 5, 2018.

\section{Introduction}

The ultraviolet (UV) wavelength regime is typically divided into four wavelength ranges, i.e., UV-A (320 to $400 \mathrm{~nm}$ ), UV-B (280 to $320 \mathrm{~nm}$ ), UV-C (200 to $280 \mathrm{~nm}$ ), and vacuum-UV (10 to $200 \mathrm{~nm}$ ). Across these ranges, there are huge needs to realize UV-based photonic and electronic devices, e.g., laser diodes (LDs), light-emitting diodes (LEDs), photodetectors (PDs), high-electron-mobility transistors (HEMTs), metal-oxide-semiconductor field effect transistors, and radio-frequency switches. For instance, the market forecast for UV-LEDs is increasing from $\$ 90$ million in the year 2014 to $\$ 800$ million by the year 2020 due to the increasing demands in epoxy curing, water purification, food or medical sterilization, and new emerging applications. ${ }^{1}$ The new applications include light sources, lithography, gas or chemical or bioagent sensing, free-space nonline-of-sight communication, phototherapy, forgery detection, medical diagnostic, consumer electronics, and horticulture. ${ }^{2}$

To realize UV-based photonic and electronic devices, several wide energy bandgap materials have been utilized, e.g., group III-nitride semiconductors, diamond, $\mathrm{SiC}, \beta-\mathrm{Ga}_{2} \mathrm{O}_{3}$, and $\mathrm{ZnO}$. ${ }^{3,4}$ The group III-nitride semiconductors have been considered the key UV-material because of their advantages, e.g., wide direct bandgap, material alloying capability for heterostructure and bandgap engineering, high electron mobility, high thermal stability, high breakdown field,

*Address all correspondence to: Mohd Sharizal Alias, E-mail: drsharizal@gmail.com; Boon S. Ooi, E-mail: boon.ooi@kaust.edu.sa 
and high saturation velocity. ${ }^{5}$ Furthermore, the material can sustain harsh temperature or radiation, and the material is toxic-free. Due to the alloying capability of the group III-nitride semiconductors, various material systems including $\mathrm{AlGaN}, \mathrm{InGaN}$, and InAlGaN have been used as the active region material for UV-based LEDs, LDs, PDs, and HEMTs. ${ }^{6-9}$ Moreover, new boroncontaining III-nitride semiconductors, e.g., BN and BAIN, have emerged recently for UV-photonic and electronic devices. ${ }^{9-13}$ Nevertheless, AlGaN has been the mostly used material for UVphotonic devices because it has a large and tunable energy bandgap from 3.4 to $6.1 \mathrm{eV}$ that can cover UV-A, B, and $\mathrm{C}$ spectral ranges. Furthermore, $\mathrm{AlGaN}$ has the nearest material compatibility with the InGaN material system. This is important to capitalize on the commercial-ready platform and technology maturity stage of InGaN-based photonic and electronic devices. However, to obtain an efficient photonic device (e.g., high external quantum efficiency, $\mathrm{EQE})$, the $\mathrm{AlGaN}$ material system encounters several key challenges regarding material growth and device fabrication. Obtaining a high-quality (crack- and defects-free) AlGaN crystal growth is challenging as the crystalline quality degrades with increasing $\mathrm{Al}$ composition and the existence of economic lattice-matched substrate is limited. ${ }^{3,6,7,14,15}$ Also, the realization of high doping efficiency for the $\mathrm{p}$-doped $\mathrm{AlGaN}$ layer is challenging because of the required high activation energy $\left(E_{A}\right)$ in such a layer (e.g., Mg-dopant case). $)^{3,6,15,16}$ Moreover, in the case of AlGaN-based light emitters, the light-extraction efficiency (LEE) for AlGaN-based devices is low $(<10 \%)$ due to the anisotropic optical polarization property for Al-rich AlGaN layers and due to the high absorption of the contact layers and encapsulation materials. ${ }^{14,16}$ To address these key challenges especially for $\mathrm{AlGaN}$-based photonic devices, numerous nanophotonics schemes related to the material growth and device stucture involving nanostructured interfaces [e.g., nanowires (NWs), nanorods (NRs), and quantum disks (QDisks)] and nanofabrication approaches [e.g., substrate patterning, surface roughening, photonic crystals (PhCs), and surface-plasmon (SP)] have been proposed. $2,14,15,17$

Here, we review the progress of nanophotonics implementations using nanostructured interfaces and nanofabrication approaches for the group III-nitride semiconductors (with the main focus on $\mathrm{AlGaN}$ material) to realize efficient UV-based photonic devices. We first discuss the AlGaN-related material and device challenges, and their effects on the device efficiency briefly. We then review the progress on using various nanophotonics schemes involving nanostructured interfaces and nanofabrication approaches adopted in the group III-nitride semiconductors for UV-based photonic devices. We will provide an overview of each approach and analysis of the device improvements (e.g., EQE and LEE). Finally, we provide an overall summary and point out the future directions of related research. This review aims to provide a framework of existing nanophotonic approaches and their further development in advancing the UV-photonic devices based on the group III-nitride semiconductors.

\section{Nanophotonics Approaches for Enhancing Device Efficiencies}

Focusing on light-emitting devices, e.g., LEDs and LDs, the device performance can be typically characterized by EQE for the LEDs case and by external differential quantum efficiency (EDQE) for the LDs case. The EQE is directly proportional to the internal quantum efficiency (IQE) and LEE. The IQE is a product of the injection efficiency (IE) and radiative recombination efficiency (RRE) of a material or a device. ${ }^{7}$ As for the EDQE, it depends on IE, IQE, and mirror losses (which is indirectly associated with the LEE). ${ }^{18}$ Thus, in both the LEDs and LDs cases, IE, IQE, and LEE play significant roles in enhancing the device performance. As stated earlier, several challenges limit the efficiency of the UV-photonic devices based on AlGaN material system, e.g., difficulty in crystal growth, p-doping, and LEE. Figure 1 shows the relationship of the above and several other challenges toward the IE, IQE, and LEE of LEDs and LDs.

Most of the challenges shown in Fig. 1 will be discussed further in the following section. Briefly here, the IE limitation is mainly due to the difficulty in obtaining high conductivity of doping (especially for $p$-type) to realize efficient $p-n$ junction devices, e.g., LEDs or LDs. For example, a high $E_{A}$ (i.e., $310 \mathrm{meV}$ for $\mathrm{Al}_{0.27} \mathrm{Ga}_{0.73} \mathrm{~N}$ ) is required to dope a Mg-dopants and will increase further for higher $\mathrm{Al}$ composition. ${ }^{18}$ The low $p$-doping efficiency reduces the IE, which 


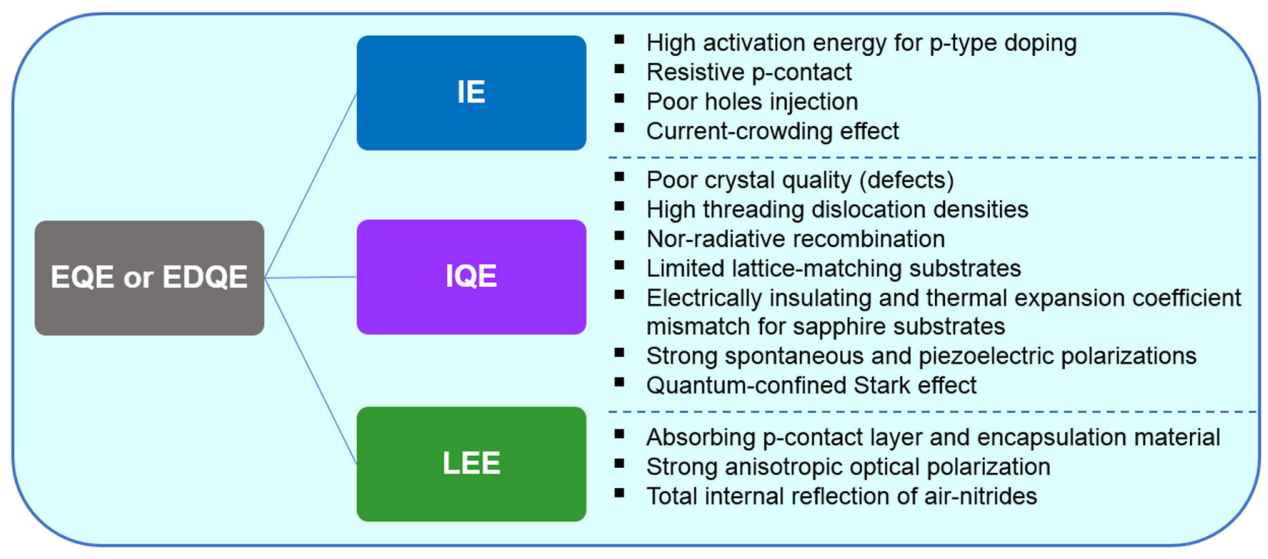

Fig. 1 A summary of most prominent challenges that limit the efficiency parameters for LEDs and LDs.

results in a high resistance $p$-contact and poor holes injection across the device. As for IQE, in general, increasing the $\mathrm{Al}$ composition to obtain shorter $\mathrm{UV}$ wavelengths in the AlGaN material causes a poor crystal quality due to increased density of defects in the form of cracks, dislocations, or impurities. Such defects can generate nonradiative center trap carriers and hence decrease the radiative recombination rate. This will reduce the IQE of the device. Due to the lack of native substrates, $\mathrm{AlGaN}$ has been grown on foreign substrates, e.g., sapphire, which is transparent to UV. However, to grow a high-quality AlGaN epitaxial structure on sapphire substrates is challenging due to large lattice mismatch and thermal expansion coefficient mismatch between the AlGaN/sapphire interface. Cracks easily formed with thicker AlGaN epilayer due to the accumulation of strong tensile strain. Compared with the InGaN epilayer on sapphire, AlGaN produced higher threading-dislocation densities (TDDs), i.e., $\sim 10^{10}$ to $10^{11} \mathrm{~cm}^{-2}$ due to the lattice mismatch, together with the large sticking coefficient and low surface mobility of $\mathrm{Al}$ adatoms during the crystal growth (in comparison, the TDDs for InGaN is $\left.\sim 10^{8} \mathrm{~cm}^{-2}\right){ }^{16,19} \mathrm{~A}$ recent approach of using bulk AlN substrates to grow AlGaN has been promising to reduce TDDs. However, the cost of AlN substrates is still expensive, and similar to sapphire, it is electrically insulating. To overcome this issue, $p$ - and $n$-contacts of the AlGaN-based devices need to be fabricated on the same side of the wafer (lateral injection). This results in nonuniform current injection across the device (current crowding) and localized heating. Furthermore, in Al-rich AlGaN epilayer, $\mathrm{O}_{2}$ can be easily incorporated into the lattice, hence increasing the impurities. ${ }^{20}$ The spontaneous and piezoelectric polarizations are dominant in group III-nitride semiconductors, and these polarizations are even stronger with higher Al composition (strain from the tensile stress). ${ }^{19}$ Both polarizations can reduce the RRE due to the quantum-confined Stark effect (QCSE), i.e., electron-hole recombination decreased from the band bending effect. ${ }^{19}$ Also, the LEE of AlGaN light-emitters suffers from a strong anisotropic optical polarization, i.e., the transverse magnetic (TM) polarization becomes dominant with increasing Al composition. Moreover, the p-contact layer (mostly p-GaN is used because of high conductivity and easy to form ohmic contact) and encapsulation materials are absorbing at most of the UV spectral range.

To overcome the above-mentioned challenges to enhance IE, IQE, and LEE for AlGaN-based UV photonic devices, different approaches using nanophotonic schemes have been developed. Figure 2 listed the nanophotonic schemes that can be applied to enhance the efficiency of UV-photonic devices. We categorized these schemes into two main parts, i.e., nanostructured interfaces and nanofabrication approaches. Using a generic UV-LD device structure as an example (as shown in Fig. 3), we list some of the nanophotonic schemes that can be used to improve the device efficiency. For example, the use of nanostructured interfaces (e.g., NWs, NRs, and QDisks) and nanofabrication approaches (e.g., substrate patterning, PhCs, and naogratings) can enhance the IQE and LEE of the device. The detail of the nanophotonic schemes and related device performances will be reviewed next. 


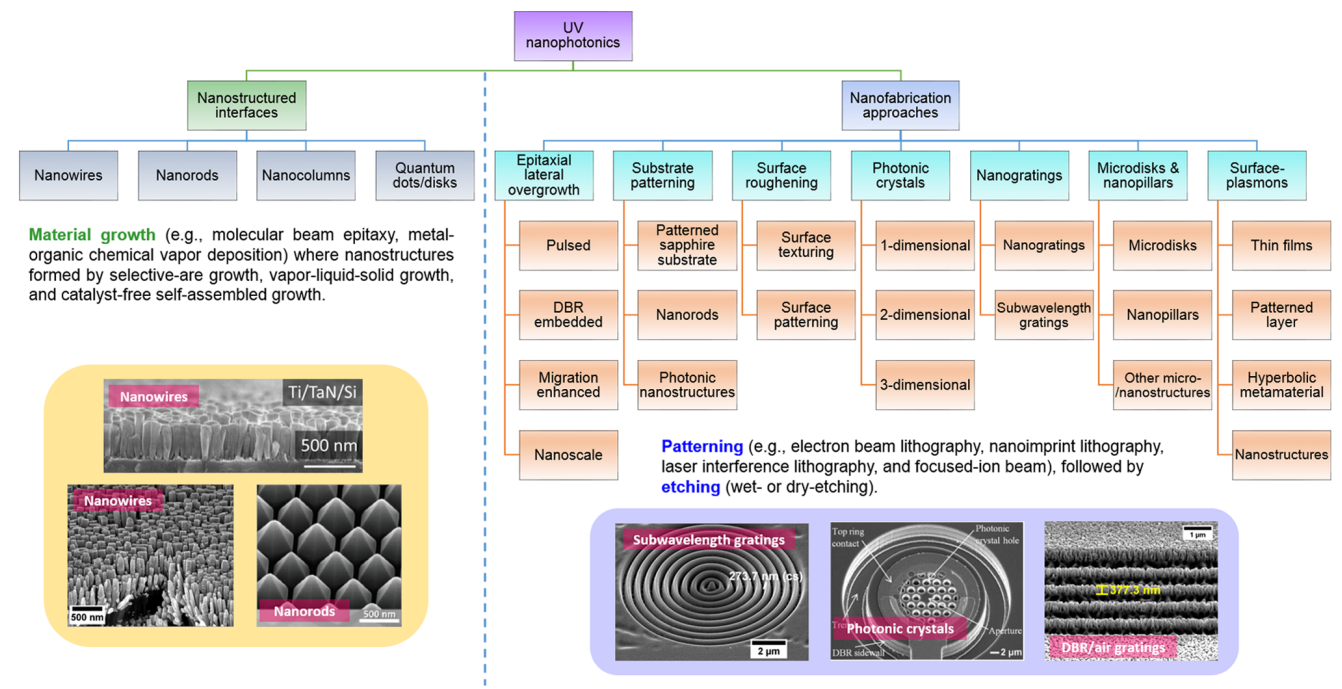

Fig. 2 Classification of nanophotonic schemes into nanostructured interfaces and nanofabrication approaches. The insets show microscopy images from several schemes of the nanophotonic.

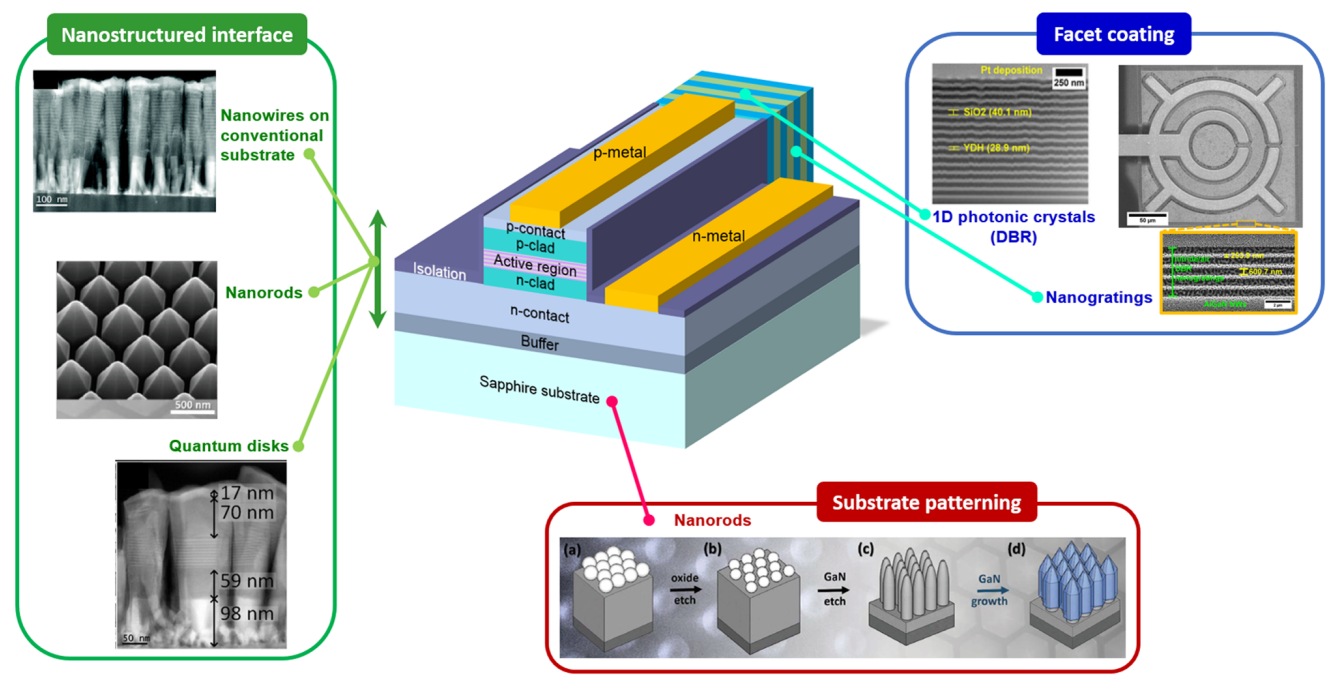

Fig. 3 Examples of nanophotonic schemes that can be applied on UV-LD to improve IQE and LEE.

\section{Nanophotonics Schemes Using Nanostructured Interfaces}

Several of the nanophotonic schemes, e.g., NWs, NRs, NCs, and monolayer are based on a bottomup approach, i.e., material growth. Due to the high crystallinity requirement for the group IIInitride semiconductors including $\mathrm{AlGaN}$, their epitaxial structures have been grown mostly using molecular beam epitaxy (MBE) and metal-organic chemical vapor deposition (MOCVD).

\subsection{Nanostructures: Nanowires, Nanorods, and Nanocolumns}

The NWs technology has recently pursued as NWs exhibit unique characteristics, e.g., high crystalline quality with low defect density and large surface-to-volume ratio. There are three significant ways to grow such high-quality NWs: the so-called lithography-based selective area growth (SAG), vapor liquid solid (VLS) growth, and the catalyst-free self-assisted growth schemes. Although the researchers have been succeeded to grow GaN NWs using VLS process, unavoidable contamination due to the metal usage in the growth process remained challenging. 
To overcome such a problem, many efforts have been explored for NWs growth in the absence of metal particles. ${ }^{21}$ In view of this, researchers have used selective-area metal-organic vapor phase epitaxy to grow ordered arrays of III-nitride NWs. ${ }^{22}$ On the other hand, the SAG and VLS growth schemes are time-consuming, complicated, lack of control over doping densities, and limited crystalline quality. ${ }^{23,24}$ An alternative growth scheme has been utilized to accomplish highdensity self-assembled defect-free group-III nitride NWs at N-rich conditions. ${ }^{25-30}$

\subsubsection{Selective area growth}

Several efforts have been made to grow high-quality GaN NWs, NRs, and NCs on patterned substrates using MOCVD and hydride vapor phase epitaxy. MBE can also be utilized to achieve SAG under enhanced nucleation of III-nitride nanocrystals that were formed through the surface nitridation of an array of group-III metal nanodisks (NDs) on $\mathrm{Si}^{24} \mathrm{At}$ low nucleation process of MBE, the III-metal sources readily adhere to an SAG mask of $\mathrm{SiO}_{2}$, which results in a formation of a large number of self-assembled GaN NCs on counterparts of the patterned area. ${ }^{31}$ For instance, Gotschke et al. have shown MBE growth of regular arrays of uniform-size GaN NWs on patterned $\mathrm{Si} / \mathrm{AlN} / \mathrm{Si}(111)$ substrates. The Si top layer has been patterned with e-beam lithography (EBL), resulting in uniform arrays of holes with different diameters and periods. ${ }^{32}$ Choi et al. ${ }^{33}$ reported the SAG of Ga-polar thin GaN NWs on patterned GaN/sapphire (0001) substrates using MOCVD with a continuous gas supply. GaN NRs formation on Ga-polar GaN by continuous mode MOCVD SAG was demonstrated under a relatively Ga-rich conditions. The growth rates of the semipolar planes, usually slowest growing planes, were controlled by varying the V/III ratio, which later allowed to grow GaN NRs along c-direction with nonpolar sidewalls.

First, the authors used a $\mathrm{GaN} / \mathrm{c}-\mathrm{Al}_{2} \mathrm{O}_{3}$ wafer that was patterned with a hexagonal array of circular holes using EBL and amorphous $\mathrm{SiN}_{\mathrm{x}}$ formed by plasma-enhanced chemical vapor deposition (PECVD) as a masking material. ${ }^{34}$ Later, $\mathrm{GaN}$ nanostructures were grown at a fixed temperature $\left(1125^{\circ} \mathrm{C}\right)$ and TMG flow rate $(24 \mathrm{sccm})$, whereas the $\mathrm{NH}_{3}$ flow rate was varied from 150 to $5 \mathrm{sccm}$. Figures 4(a)-4(d) show scanning electron microscope (SEM) images for the evolution of the $\mathrm{GaN}$ nanostructure morphology as a function of $\mathrm{NH}_{3}$ flow rate of $150,25,10$, and $5 \mathrm{sccm}$, respectively. This revealed that the nanopyramids (NPs) structure
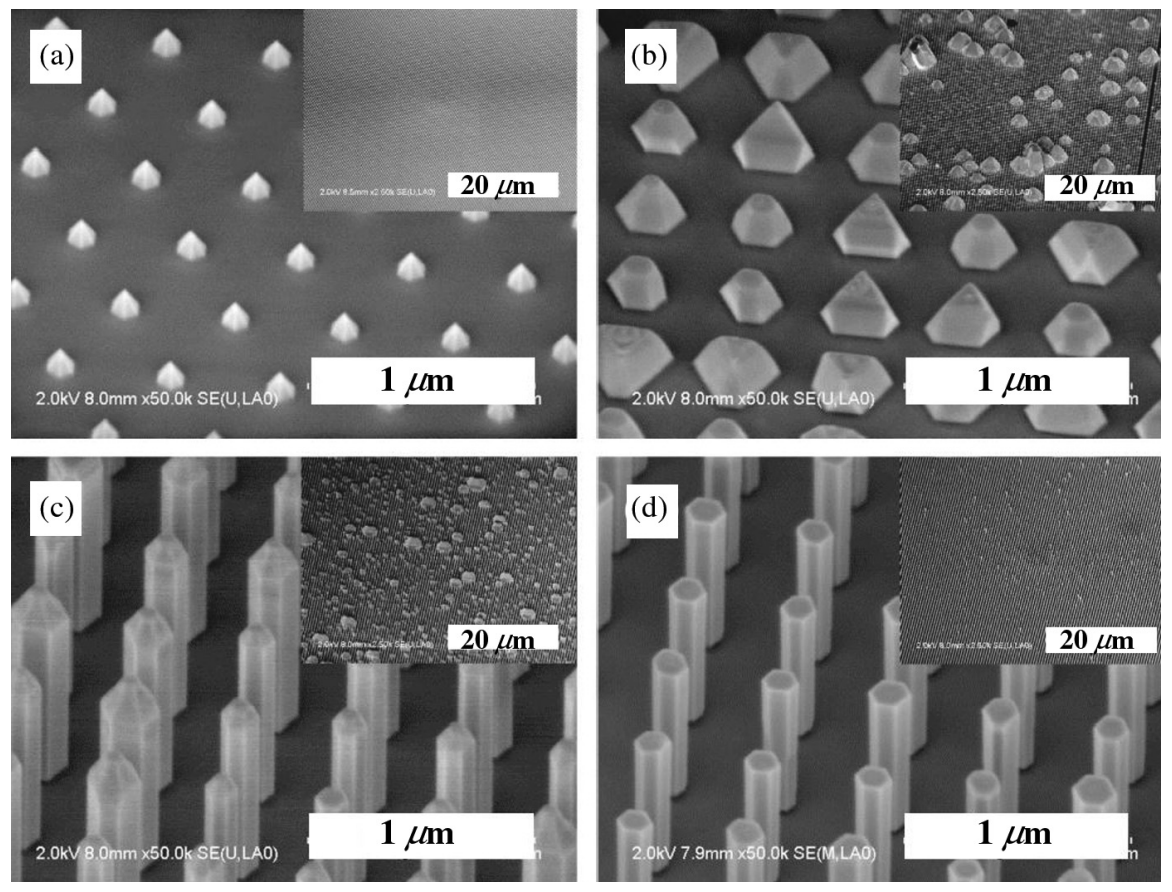

Fig. $4 \mathrm{GaN}$ nanostructures evolution with respect to $\mathrm{NH}_{3}$ flow rate show in (a) 150, (b) 25, (c) 10 , and (d) $5 \mathrm{sccm}$. The insets show lower magnification SEM images. Reproduced with permission from Ref. 35. 


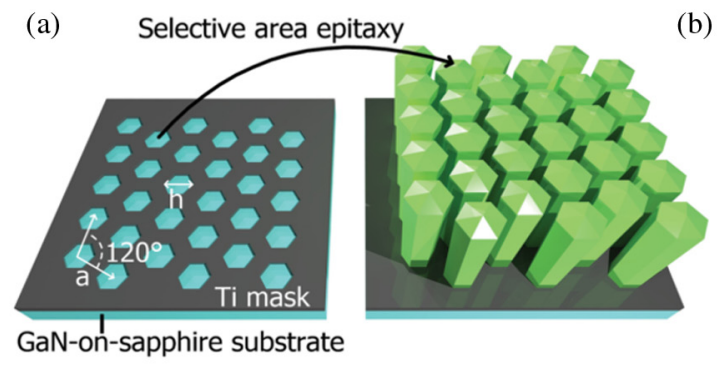

(b)

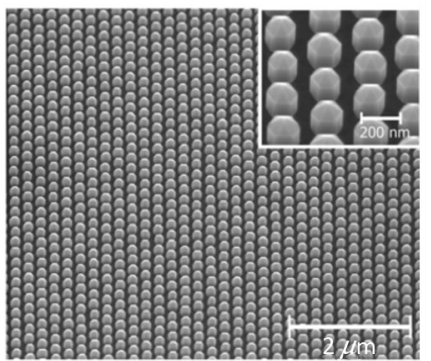

Fig. 5 (a) Left: arrays of nanoscale opening apertures defined by EBL process on a Ti mask on planar GaN template on the c-plane sapphire substrate. Right: schematic of the SAG of GaN NWs arrays on the nanopatterned substrate. (b) A tilted view SEM image showing GaN NWs arrays selectively grown in the opening apertures. The inset shows a high-magnification SEM image of GaN NWs arrays. Reproduced with permission from Ref. 36.

defined by $\{1-101\}$ semipolar planes need to be formed under the higher pressure of $\mathrm{NH}_{3}$. As the size of the NPs is observed to be comparable with the original opening diameter, the growth rate of semipolar $m$-plane $\{1-101\}$ is extremely low, which determines the formation of nanostructures as NPs.

The SAG of GaN NWs was further utilized as a template to grow AlGaN NWs-based photonic devices. For example, Le et al. ${ }^{36}$ have used arrays of nanoscale opening apertures defined by EBL process on a Ti mask on planar GaN template on the c-plane sapphire substrate to grow GaN NWs. Later, this approach has been used as a template to grow defect-free coalesced AlGaN NWs-based UV-LED device structures.

Figure 5(a) shows the schematic representation of selective area epitaxy of GaN/AlGaN NWs arrays on a GaN template/c-sapphire substrate by employing a thin $(\approx 10 \mathrm{~nm})$ Ti layer as the growth mask. Hexagonal openings situated as a triangular lattice were created on the $\mathrm{Ti}$ mask having a lattice spacing, $a$ and a lateral size, $h$ for each opening. These nanohole arrays were then created on the Ti mask using EBL and reactive ion etching. Later, GaN NWs arrays were selectively grown in the opening apertures by MBE. Figure 5(b) shows the SEM image of GaN NWs, exhibiting a high degree of size uniformity and are vertically aligned along the $c$-axis with well-defined hexagonal pyramid morphology comprised of six semipolar top facets.

\subsubsection{Vapor-liquid-solid growth}

The growth of III-V nanostructures has also been achieved using catalyst-assisted routes, e.g., catalytic metal-induced VLS mechanism, where metal droplets enable the material to solidify below the frozen liquid-solid interface. ${ }^{37,38}$ The nanostructures can be then formed via the vapor-liquid-solid mechanism with, e.g., $\mathrm{Au}, \mathrm{Fe}$, or $\mathrm{Ni}$ as growth initiators. ${ }^{39}$ Recently, Sivadasan et al. ${ }^{40}$ reported on the optical properties of AlGaN NWs grown using the CVD method. They investigated the influence of the distribution of $\mathrm{Au}$ catalyst nanoparticles on the size and shape of AlGaN NWs. However, due to the deleterious effects of metal impurities on the structural, optical, and electrical properties of the semiconducting nanostructures grown using the VLS process, ${ }^{23}$ researchers seldom used this method for the AlGaN NWs growth.

\subsubsection{Catalyst-free self-assembled nanowires on conventional substrates}

Another method to grow NWs is self-assembled growth, which does not require any catalyst and prepatterned substrates. Several researcher works have been reported on the growth of such spontaneously formed NWs on Si substrates using the MBE and MOCVD. ${ }^{28,30,41}$ As compared with the ELOG, VLS, and SAG growth schemes, employing MBE or MOCVD for NWs growth is less time consuming and more scalable. Under certain conditions, e.g., N-rich and low metal conditions (V/III ratio $\gg 1)$ the growth of self-assisted dislocation free NWs on sapphire and Si substrates can be realized.

In this context, Janjua et al. ${ }^{42}$ reported AlGaN NWs LEDs using the pendeo-epitaxy technique. Highly dense and spontaneously coalesced NWs emitting at 303-nm wavelength were 

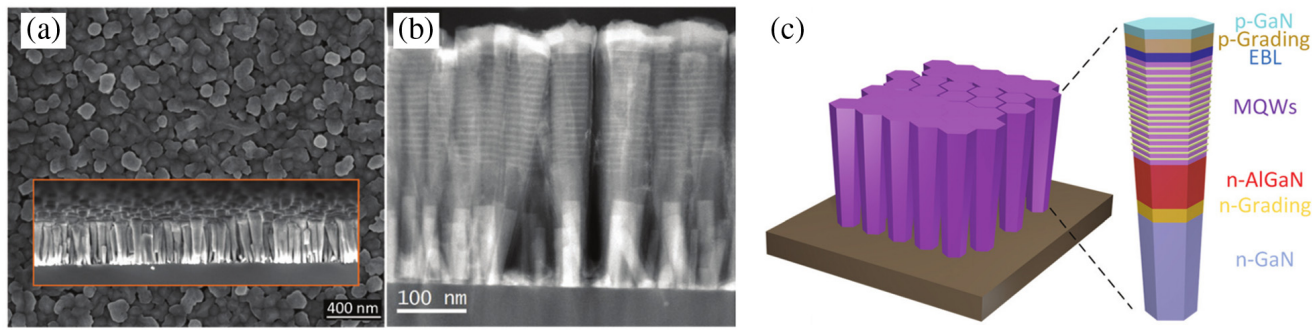

Fig. 6 (a) Top-view SEM image of the AIGaN NWs-based device structure. The inset shows cross-sectional SEM of these NWs. (b) STEM image of the coalesced AIGaN NWs. (c) A schematic of the device. Reproduced with permission from Ref. 42.

grown on Si substrates. Figure 6(a) shows a top-view SEM image of the AlGaN NWs, whereas the inset shows a cross-sectional SEM of these NWs. The coalescence of the top p-GaN (in the inset) improves the current spreading and injection and reduces carrier leakage without the needs for planarization. Figure 6(b) shows a cross-sectional scanning tunneling electron microscope (STEM) image of an array of AlGaN NWs with device schematic shown in Fig. 6(c). To further optimize the AlGaN NWs LEDs, the self-assembled NWs were grown with varying QDisks thickness to study the carrier separation in the active-region and implemented a graded $p$-contact-layer to increase the output power. ${ }^{43}$ They observed an improvement by 100 times in the direct recombination rate for samples with thicker QDisks thickness of $\sim 5$ monolayers (MLs) compared with the sample with $\sim 2$ MLs-thick QDisks. Moreover, the sample with graded top $\mathrm{Mg}$-doped $\mathrm{AlGaN}$ layer shows 10 times improvement in the output power.

In addition to LEDs, NWs-based LD has been recently demonstrated. Li et al. ${ }^{44}$ reported a highly stable and electrically pumped lasing emission in the UV-A at low temperature using the defect-free disordered AlGaN core-shell NWs arrays grown on Si substrate. Figure 7(a) shows

(a)

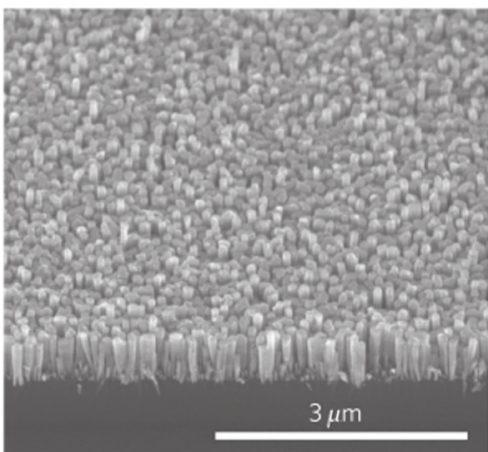

(c)

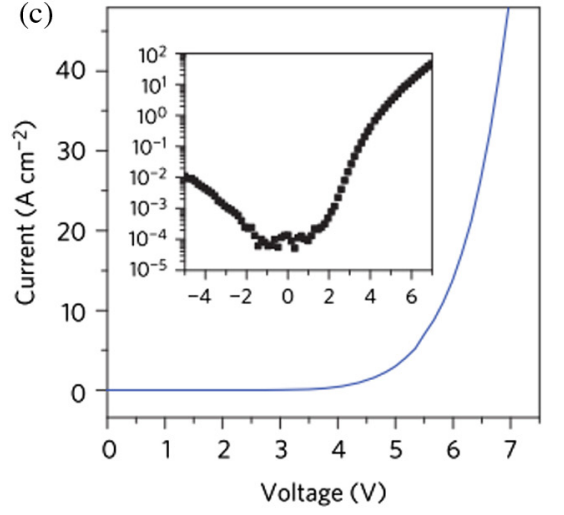

(b)
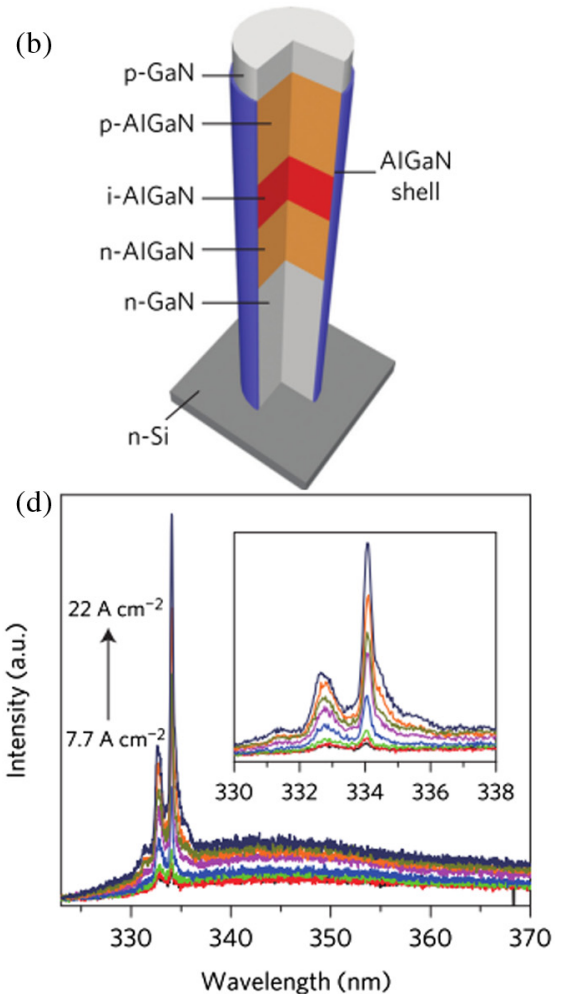

Fig. 7 (a) SEM image of NWs grown on a Si substrate, (b) schematic of the device, (c) I-V characteristics of the device; the inset shows the I-V curve on a semilog scale, and (d) emission spectra measured at $6 \mathrm{~K}$ under different current densities with the inset shows zoom-in of the lasing spectra. Reproduced with permission from Ref. 44. 

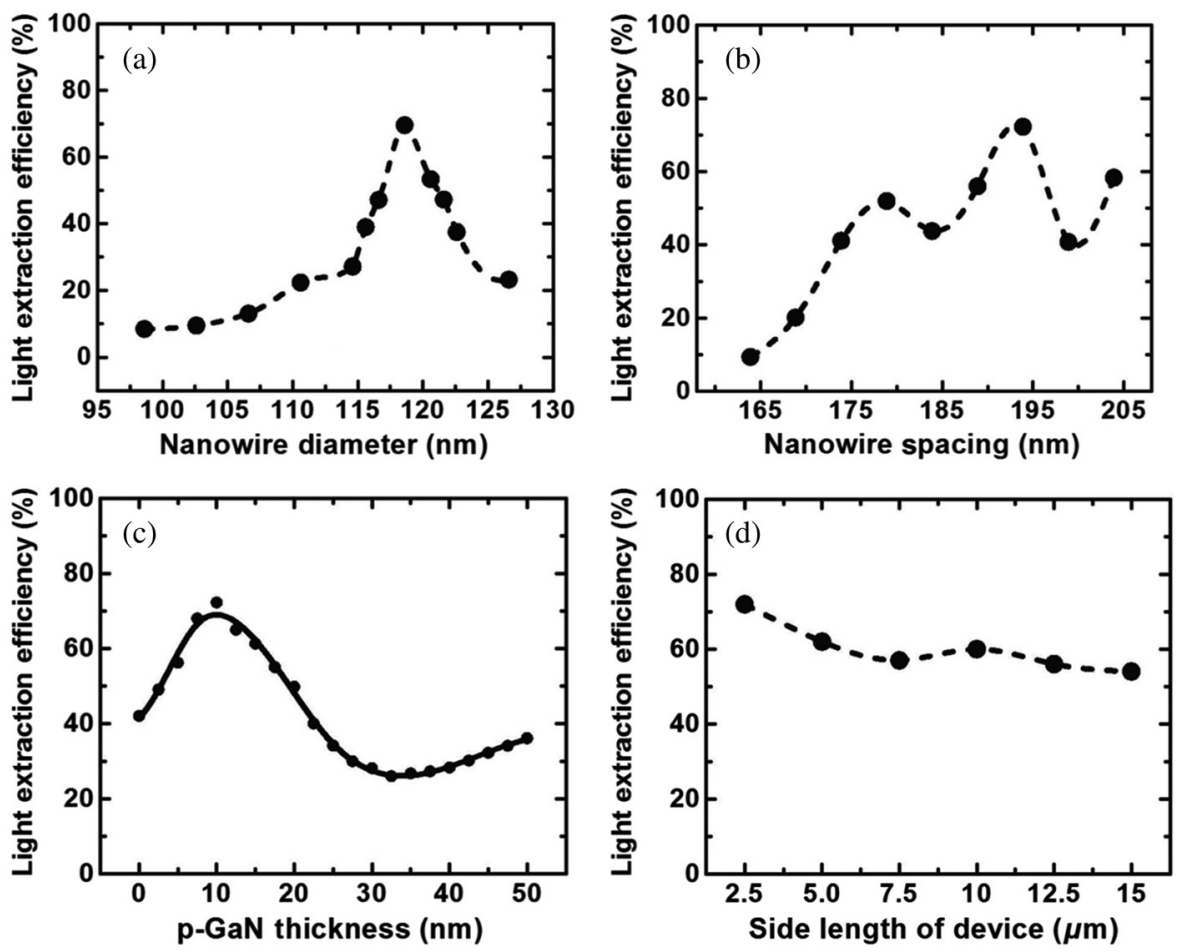

Fig. 8 (a) LEE versus diameter of NWs, (b) LEE versus spacing of NWs, (c) LEE versus thickness of the p-GaN layer, and (d) simulated LEE for devices with side length variations. Reproduced with permission from Ref. 45.

the SEM image of NWs grown on a Si substrate with the device schematic shown in Fig. 7(b). Figure 7(c) shows the I-V curve with the inset indicating a low leakage current under reverse bias. The lasing characteristics of $\mathrm{AlGaN}$ NWs were investigated by electrical injection under a continuous-wave operation at various temperatures. Figure 7(d) shows the EL spectra measured at $6 \mathrm{~K}$. Two discrete peaks centered at 332.7 and $334.1 \mathrm{~nm}$ were observed. The spectral linewidth is as narrow as $0.2 \mathrm{~nm}$ above the lasing threshold of $\sim 12 \mathrm{~A} \mathrm{~cm}^{-2}$.

Using NWs, an enhancement in the LEE for a UV-LED can be achieved. Djavid and $\mathrm{Mi}^{45}$ studied the lateral side emission properties of NWs UV-LEDs using a simulation approach. Enhanced LEE of $\sim 72 \%$ was accomplished by optimizing the NWs size and density, as well as optimizing the thickness of the p-GaN layer. Figure 8(a) shows the dependence of LEE on the diameter of NWs. Hence, it is evident that there is an optimum diameter at which the light could be efficiently coupled through the NWs for efficient lateral side emission. Figure 8(b) shows the LEE that was further calculated as a function of the spacing between NWs from 163 to $203 \mathrm{~nm}$. The corresponding LEE varied from 9\% to 72\%. TM polarized light can propagate horizontally through the NWs arrays resulting in significantly enhanced LEE. Figure 8(c) shows the LEE having an overall decreasing trend with increasing $\mathrm{p}-\mathrm{GaN}$ thickness. Also, the authors have performed a simulation with device sizes in the ranges of $2.5 \times 2.5$ to $15 \times 15 \mu \mathrm{m}^{2}$. As shown in Fig. 8(d), the LEE decreases from $72 \%$ to $54 \%$ as device size increases. In addition to the diameter and spacing of NWs, the tapering angle of the grown AlGaN NWs can also enhance the LEE for a UV-LED. ${ }^{46}$

\subsection{Quantum Disks and Quantum Dots}

Here, we discuss the research progress on the use of quantum dots (QDs) and QDisks for UV photonics. First, we focus more on the material properties and then we will discuss the advancement of the device based on QDs and QDisks.

In addition to the ability to tune the energy bandgap of the group III-nitride semiconductors by material composition variation, this tuning can also be achieved through quantum 
(a)
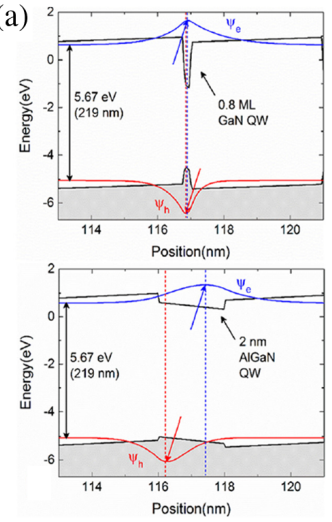

(b)

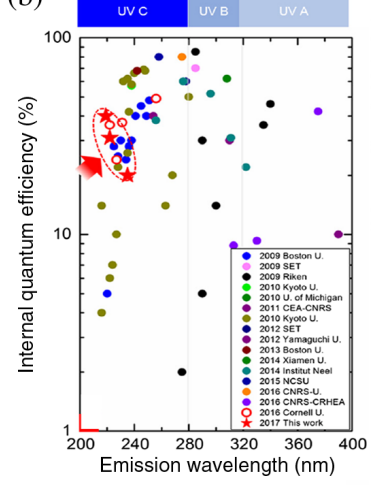

(c)

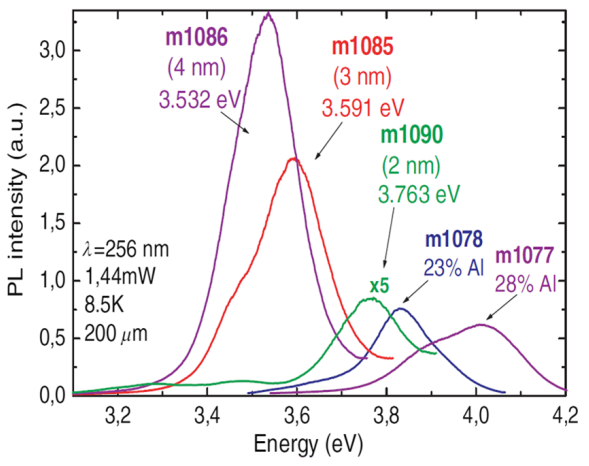

Fig. 9 (a) Enhancement of IQE using GaN QWs due to the reduced QCSE. Reproduced with permission from Ref. 47. (b) Comparison of measured IQE of GaN- and AIGaN-based heterostructures. Reproduced with permission from Ref. 47. (c) PL spectra of GaN QDisks with thickness variation. Reproduced with permission from Ref. 48.

confinement using a thin active layer in the form of QDs and QDisks structures. Islam et al. ${ }^{47}$ have shown that by changing the thickness of GaN QDisks, a PL emission as short as 219-nm wavelength can be obtained as shown in Fig. 9(a). Recent works reveal that a higher IQE can be achieved in the GaN-based QDs ${ }^{49,50}$ and QDisks ${ }^{47,48,51,52}$ compared with the AlGaN-based quantum wells structure in the similar wavelength range. Figure 9(b) shows a comparison between $\mathrm{GaN}$ and $\mathrm{AlGaN}$ heterostructures in terms of IQE. ${ }^{47}$

Tanaka et al. ${ }^{53}$ grew GaN QDs on AlGaN-layered surfaces with MOCVD and observed a strong PL peak at $\sim 3.55 \mathrm{eV}$. In the same year, Seifert et al. ${ }^{54}$ demonstrated the growth of III-V and III-nitrides-based QDs by means of the Stranski-Krastanov method using the MOCVD platform. This is followed by the successful growth of GaN QDs using the MBE platform. ${ }^{55}$ Next, Ristić et al. ${ }^{56}$ reported defect-free and fully relaxed AlGaN NCs on Si (111) substrate grown by MBE. In contrast, GaN QDisks (also grown for a comparison study) are fully strained as indicated by the Raman measurement. ${ }^{56} \mathrm{~A}$ comprehensive study on optical, electrical, and morphological properties of GaN QDisks was later followed by Ristić et al. ${ }^{48,57}$ It was found that PL emission red-shifted (due to the QCSE) when the Al composition in the GaN/AlGaN QDisks is increased from $20 \%$ to $28 \%$. However, blue-shift in PL emission was observed [as shown in Fig. 9(c)] when the thickness of QDisks is decreased from 4 to $2 \mathrm{~nm}$ (using the set of NCs with $28 \%$ of $\mathrm{Al}$ composition with different QDisks thickness). ${ }^{48,57}$

Recently, several UV-photonic devices were demonstrated based on the GaN QDs and QDisks. ${ }^{42,49,50,51,58-63}$ One of the challenges to achieving efficient UV emitting devices is to overcome the poor current injection. In this context, Verma et al. ${ }^{49,50}$ demonstrated UV-LED based on

(a)

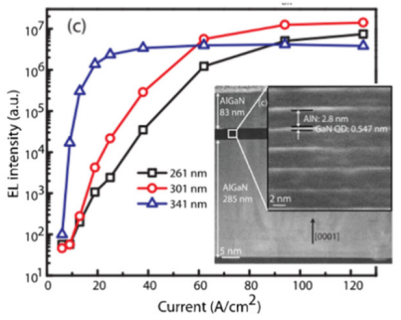

(b)

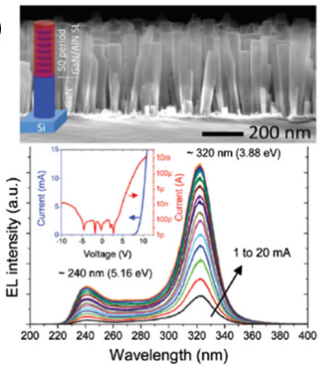

(c)

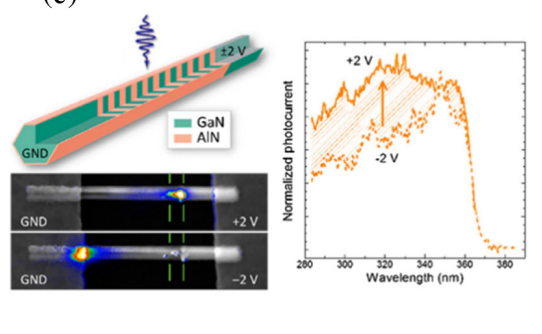

Fig. 10 (a) EL characteristic and cross-sectional STEM (inset) for GaN QDs UV-LED. Reproduced with permission from Ref. 49. (b) Cross-sectional SEM and EL characteristic (I-V curve inset) for GaN QDisks UV-LED. Reproduced with permission from Ref. 51. (c) A schematic of the GaN QDisks-based UV-PD, heat maps (under biased) overlaid with the SEM images and spectral response under several voltage biases. Reproduced with permission from Ref. 63. 
Alias et al.: Review of nanophotonics approaches using nanostructures...

Table 1 A summary of research for material and devices based on GaN QDs and QDisks.

\begin{tabular}{|c|c|c|c|c|}
\hline Device & Active region & $\begin{array}{l}\text { Peak emission } \\
\text { wavelength }(\mathrm{nm})\end{array}$ & Remarks & Ref. \\
\hline \multirow[t]{5}{*}{ PL structure } & GaN QDisks & 219 & IQE $\sim 40 \%$ & 47 \\
\hline & GaN QDisks & 222 to 231 & IQE 35\% & 52 \\
\hline & GaN QDs & 295 to 326 & - & 64 \\
\hline & GaN QDisks & 358 to 306 & - & 48 and 57 \\
\hline & GaN QDs & 350 & - & 53 \\
\hline \multirow[t]{4}{*}{ LED } & GaN QDisks & 242 & $\mathrm{EQE} \sim 0.012 \%$ & 65 \\
\hline & GaN QDisks & 240 & - & 51 \\
\hline & AIGaN QDisks & 325 & - & 62 \\
\hline & AlGaN QDisks & 337 & - & 42 \\
\hline \multirow[t]{2}{*}{ PD } & GaN QDisks & 280 to 360 & - & 63 \\
\hline & GaN QDisks & 300 & Response $\sim 2 \times 10^{3} \mathrm{~A} / \mathrm{W}$ & 61 \\
\hline
\end{tabular}

GaN QDs as an active layer and utilized tunnel junction (TJ) scheme. Figure 10(a) shows the cross-sectional STEM of the device with the EL characteristic. In Fig. 10(b), Sarwar et al. ${ }^{51}$ demonstrated GaN-based LED utilizing QDisks at 1-ML-thick embedded in AIN NW on a Si substrate with EL emission at sub-250-nm wavelength. In addition to UV-LEDs, several PDs based on GaN QDisks have been reported recently. ${ }^{61,63}$ For example, Spies et al. ${ }^{63}$ presented a study in GaN/AIN QDisks embedded in a single GaN NW as shown in Fig. 10(c). With such a structure, they claimed to enhance the response under positive/negative bias in the UV-A and UV-B regimes [Fig. 10(c)].

To summarize, the ongoing research on GaN-based QDs and QDisks is paving a promising path to develop highly efficient UV-photonic devices. Table 1 summarizes the findings of research performed so far for both the material optimization and devices development.

\section{Nanophotonics Schemes Using Nanofabrication Approaches}

Various nanophotonic approaches to enhance the material properties (IQE) and device performance (e.g., IE, LEE, and EQE) of UV-photonic devices are discussed in this section. The nanophotonic approaches can be realized using advanced nanofabrication techniques including (1) lithographic patterning, e.g., EBL, nanoimprint lithography (NIL), and laser interference lithography (LIL), followed by wet- or dry-etching, or (2) direct patterning/etching using focused-ion-beam (FIB).

\subsection{Epitaxial Lateral Overgrowth}

Most common obstacles in the growth of group-III nitride semiconductors are the lattice/thermal mismatch between the grown epilayers and foreign substrates (due to the lack of native substrates that results in high TDDs $\left(10^{7}\right.$ to $\left.10^{9} \mathrm{~cm}^{-2}\right)$. Thus, a key factor to improve the performance of UV-devices is to reduce the TDDs and to solve the cracking issue of AlN and AlGaN films that typically originate from tensile strain beyond the critical layer thickness. Considering the cost and large area limitations of the native substrates (e.g., AlN or GaN), the devices that are grown on the native substrates are difficult to be commercialized. Therefore, to enhance the material quality of epilayers grown on nonnative substrates, different approaches, e.g., epitaxial lateral overgrowth (ELOG), pulsed-ELOG (PLOG), migration-enhanced ELOG (MEELOG), patterned dielectric distributed Bragg reflector (DBR) embedded ELOG, and nanoscale-ELOG schemes, 
have been suggested and successfully employed. In the ELOG growth scheme, a masked substrate is used in such a way that the material selectively grows on the unmasked region and thereby lateral coalescence takes place at the growth fronts.

For instance, Balakrishnan et al. ${ }^{66}$ have demonstrated an air bridge-assisted high-temperature ELOG approach to deposit $12-\mu \mathrm{m}$-thick, high-quality AlN layers over a patterned $6 \mathrm{H}-\mathrm{SiC}$ substrate as templates for the UV-LED. The overall dislocation density of the layer was estimated to be in the range of $10^{6} \mathrm{~cm}^{-2}$ or less with major portions of the layer formed over the voids are dislocation free. Chen et al. ${ }^{67}$ reported on pulsed-ELOG (PLOG) of AlN films on basal plane sapphire substrates. This approach, at temperatures higher than $1150^{\circ} \mathrm{C}$, enhanced the adatom migration, thereby significantly increasing the lateral growth rates. This enabled a full coalescence in wing regions as wide as 4 to $10 \mu \mathrm{m}$ resulting in a reduction of the TDDs from $10^{10}$ to $10^{7} \mathrm{~cm}^{-2}$. Moreover, Adivarahan et al. ${ }^{68}$ reported the growth of LED structures with AlGaN multiple quantum well (MQW) on a 17- $\mu$ m-thick, high-quality PLOG AlN layers/sapphire [Figs. 11(a) and 11(b)] and obtained UV emission at $290 \mathrm{~nm}$ [Fig. 11(c)].

Figure 11(d) shows comparison between the output power of standard and PLOG grew UVLEDs; the output powers of PLOG AlN-based LED devices do not saturate for DC pump currents, whereas the standard LED output powers start to saturate. The superior performance of the reported UV-LEDs was ascribed to the reduced thermal impedance and the decrease of overall defect density in the laterally overgrown AlN. Additionally, Jain et al. ${ }^{69}$ reported the coalescence of growth fronts using a technique in which the migration-enhanced MOCVD was successfully combined with the ELOG method to grow AlN with less defect density.

Cheng et al. ${ }^{70}$ have demonstrated an enhancement of the output power of UV-LED by realizing ELOG on a patterned DBR substrate. The UV-LED samples were grown on c-plane sapphire substrates by a low-pressure MOCVD system. Figure 12(a) shows the schematic diagram of the fabricated UV-LED with embedded $\mathrm{HfO}_{2} / \mathrm{SiO}_{2}$ DBR. Figures 12(b) and 12(c) show the

(a)

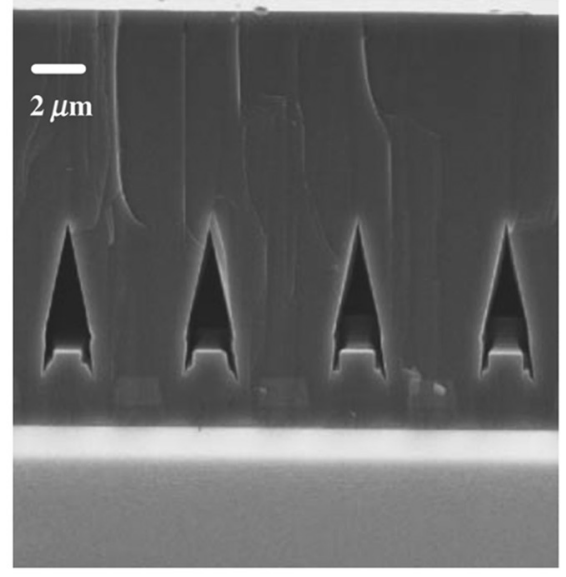

(c)

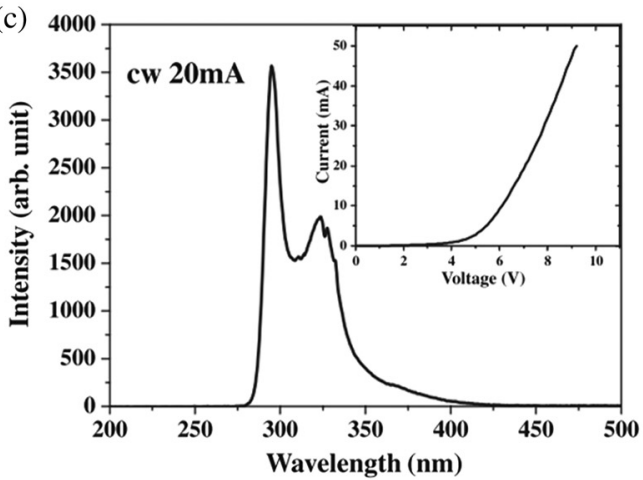

(b)

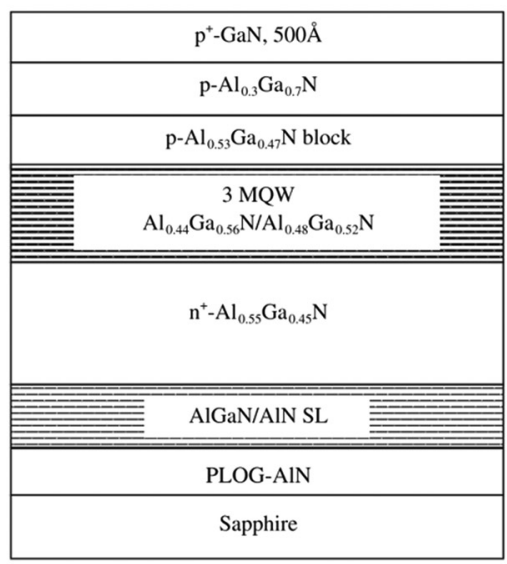

(d)

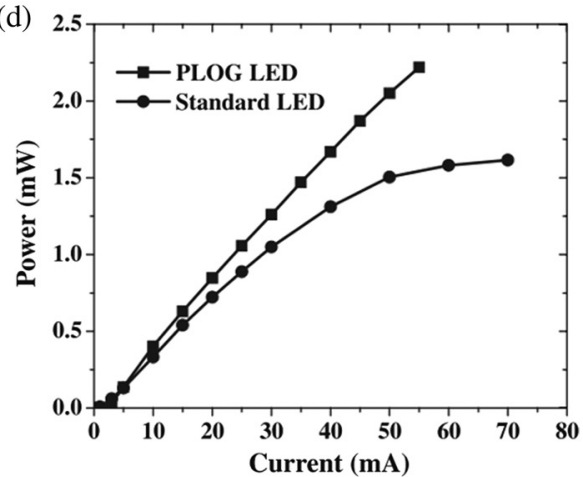

Fig. 11 (a) Cross-sectional SEM of PLOG of AIN, (b) schematic diagram of the UV-LED structure, (c) EL spectrum of the device with I-V curve (in the inset), and (d) a comparison between standard and PLOG grew LEDs. Reproduced with permission from Ref. 68. 


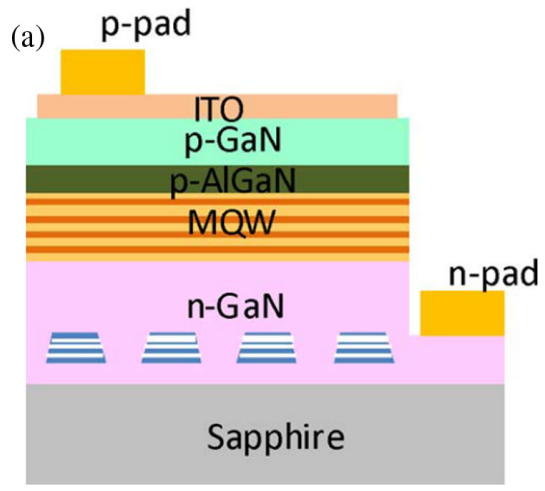

(d)

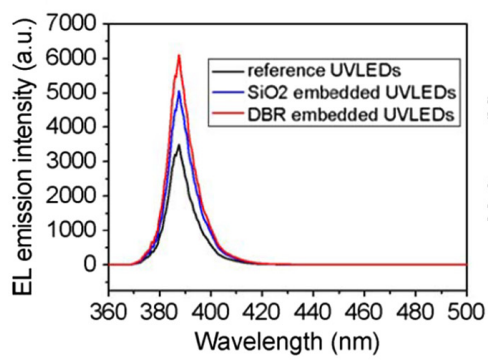

(b)

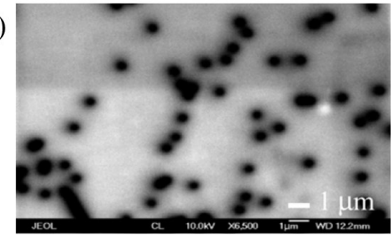

(c)

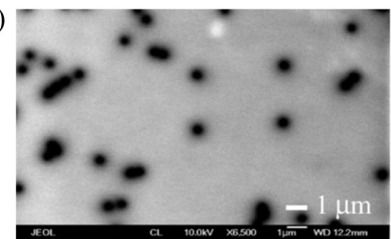

(e)

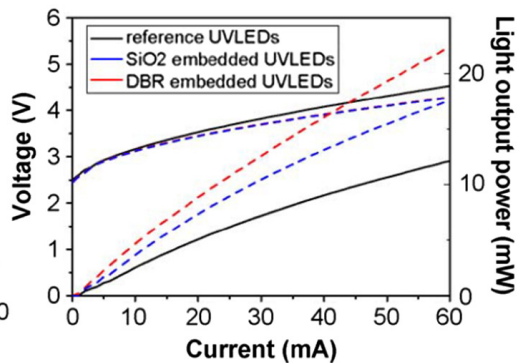

Fig. 12 (a) Schematic of ELOG using patterned DBR, (b) CL image of the reference sample, (c) CL image of the surface of DBR embedded UV-LED after MOCVD growth, (d) EL spectrum, and (e) $\mathrm{L}-\mathrm{I}-\mathrm{V}$ characteristics of all samples. Reproduced with permission from Ref. 70.

cathodoluminescent (CL) images acquired for a reference sample (without DBR) and with DBR, respectively. The dark spots denote strong nonradiative recombination centers showing low CL intensity result from the TDDs terminated at the surface. The defect density of DBR embedded UV-LED is 36\% lower compared with the reference UV-LED. Figure 12(d) shows the EL spectrum, whereas Fig. 12(e) shows the light-current-voltage (L-I-V) characteristic for all samples. The optical power of $\mathrm{SiO}_{2}$ - and DBR-embedded UV-LEDs have significant $45 \%$ and $75 \%$ improvement as compared with the reference device. This enhancement was attributed to the improvement in the material quality from ELOG and in the light output coupling from the embedded DBR. Similarly, Huang et al. ${ }^{71}$ reported the InGaN/GaN MQW UV-LED with embedded $\mathrm{Ta}_{2} \mathrm{O}_{5} / \mathrm{SiO}_{2}$ micromirror array (MMA) in the ELOG of GaN for the LEE enhancement. They achieved a 75.2\% LEE enhancement for the patterned $\mathrm{SiO}_{2} \mathrm{LED}$, and a $102.6 \% \mathrm{LEE}$ enhancement for the MMA-LED, both were compared with the control sample.

The nanoscale ELOG scheme shows a more TDDs reduction in the overgrown group IIInitride semiconductors as compared with the microscale ELOG. ${ }^{72-74}$ For example, Conroy et al. $^{73}$ reported an inexpensive nanoscale patterning process for ELOG on AlN layers grown by MOCVD. The uniform 1- $\mu \mathrm{m}$-length NRs structure, formed by dry-etching of monolayer silica spheres, was used as a template for nanoscale ELOG epitaxy. A schematic drawing and an SEM image of the structure are shown in Figs. 13(a) and 13(b), respectively. A massive reduction in TDDs in the overgrown AIN layer is observed when the sample was annealed at $1100^{\circ} \mathrm{C}$. This is formed at a much lower temperature compared with that typically required for microscale ELOG. ${ }^{75}$ Figure 13 (c) shows the FIB section of AlN overgrowth at $1100^{\circ} \mathrm{C}$ tilted to $g=(0002)$ showing $a$-type dislocations. Figures 12(e) and 12(f) show the dislocations at higher magnifications, whereas Fig. 13(d) shows the FIB cross sections of typical high-quality three step AlN templates in the $g=(0002)$ without ELOG. Without NRs patterning, the observed density of the edge and mixed dislocations was found to be $\sim 4.4 \times 10^{9} \mathrm{~cm}^{-2}$. In comparison, AIN films produced using the NRs ELOG process had TDDs of $<10^{8} \mathrm{~cm}^{-2}$.

Recently, Lee et al. ${ }^{74}$ have demonstrated AlGaN-based UV-LED with periodic air-voidsincorporated nanoscale patterns enabled by nanosphere lithography and ELOG on a sapphire substrate. Figure 14(a) shows their fabrication process of AlN/sapphire nanopatterned substrate 

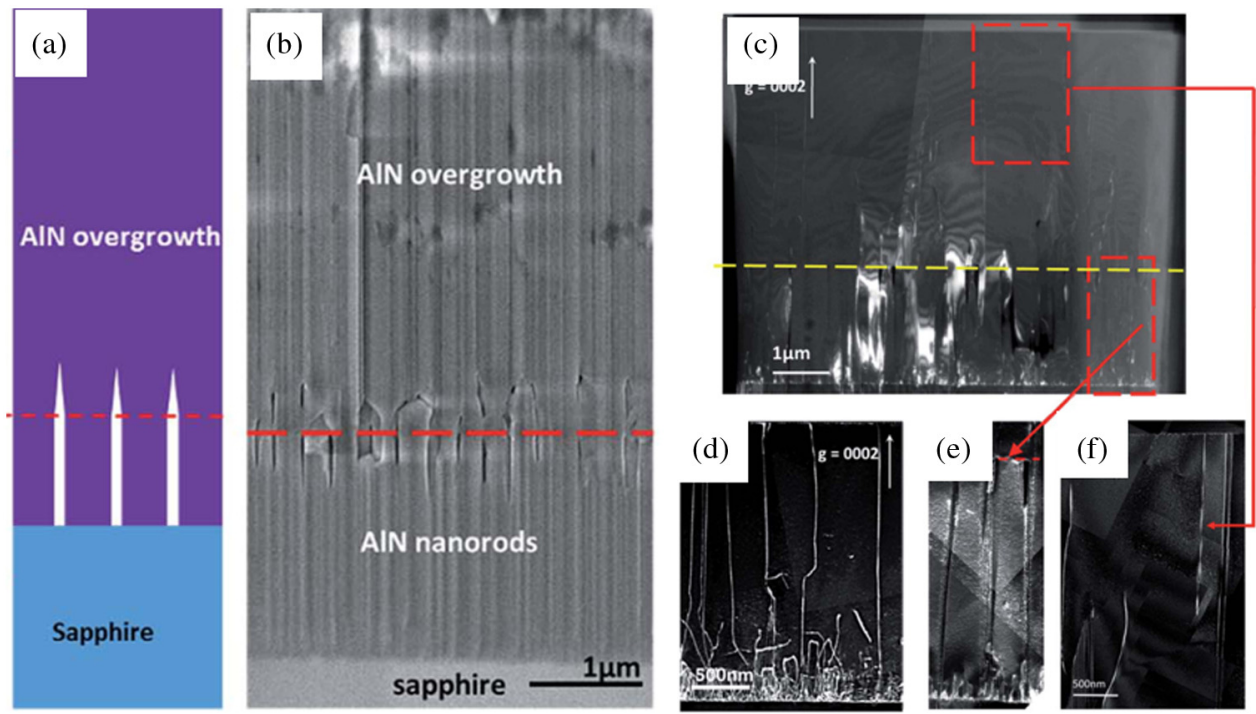

Fig. 13 (a) Schematic of the nanoscale-ELOG of AIN overgrowth on the NRs, (b) cross-sectional SEM image of the interface of AIN NRs and the overgrown layer with air gaps, (c) FIB section of AIN overgrowth at 1100 deg showing a-type dislocations, (d) cross-sectional FIB, (e) and (f) dislocations showed at higher magnifications. Reproduced with permission from Ref. 73.

(NPS). After initial growth, the self-assembled Si nanospheres as etch masks were coated on the AlN layer/c-sapphire. Then, dry-etch was accomplished to transfer the nanospheres pattern to the underlying AlN layer. Figures 14(b) and 14(c) are plan-view, and tilted view SEM images of the fabricated NPS, whereas the cross-section is shown in Fig. 14(d). Figure 13(e) shows the I-V characteristics for all devices. Figure 14(f) shows the average light output power (LOP) as a

(a)

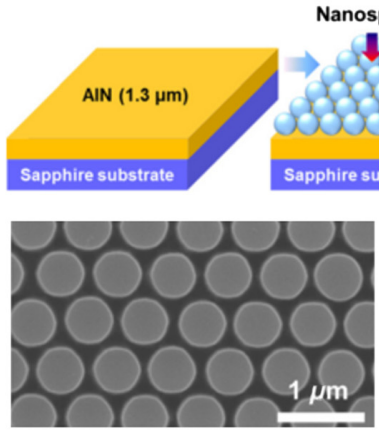

(e)

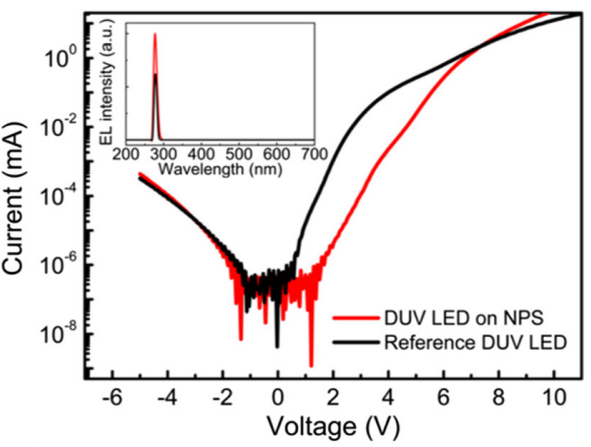

(d)

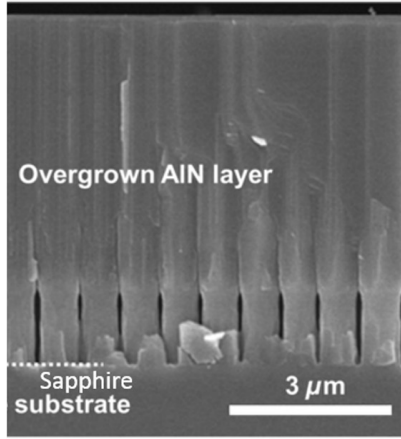

(f)

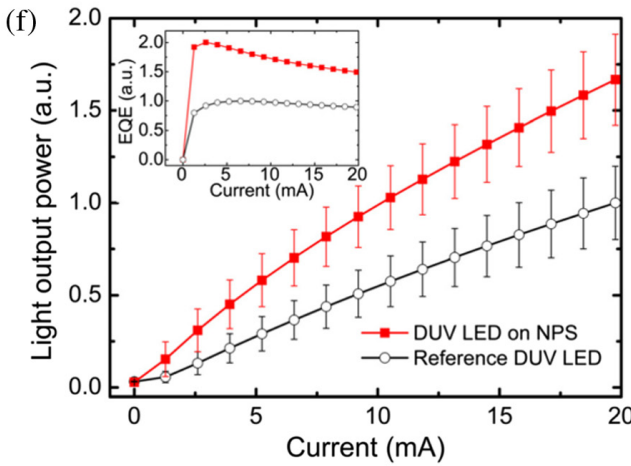

Fig. 14 (a) Schematic of silica nanosphere lithography, (b) top- and (c) tilted-view SEM images of the fabricated NPS. (d) Cross-section SEM image of the overgrown AIN layer on the NPS. (e) I-V characteristics of all devices. The inset shows EL spectra. (f) Average LOP with error bars as a function of injection current. The inset shows relative EQE. Reproduced with permission from Ref. 74. 
Alias et al.: Review of nanophotonics approaches using nanostructures...

Table 2 A summary of research using ELOG scheme to improve the UV device performance.

\begin{tabular}{lccccc}
\hline \hline Substrate & ELOG type & $\begin{array}{c}\text { TDD without } \\
\text { ELOG }\left(\mathrm{cm}^{-2}\right)\end{array}$ & $\begin{array}{c}\text { TDD with } \\
\text { ELOG }\left(\mathrm{cm}^{-2}\right)\end{array}$ & Enhancement & Ref. \\
\hline Sapphire & Pulsed & $10^{10}$ & $10^{7}$ & PL intensity (10 times) & 67 \\
Sapphire & Pulsed & - & $10^{7}$ & $(40 \%)$ relative power & 68 \\
6H-SiC & HT & - & $10^{6}$ & - & 66 \\
Sapphire & DBR embedded & $2.5 \times 10^{6}$ & $1.6 \times 10^{6}$ & $75 \%$ (EL intensity) & 70 \\
Sapphire & Hetero facet controlled & - & $3.9 \times 10^{8}$ & $8.2 \%$ (EDQE) & 76 \\
Sapphire & Nanoscale & $1.5 \times 10^{9}$ & $3 \times 10^{8}$ & $14.5 \%$ (Reflectance) & 73 \\
Sapphire & Nanoscale & $6.0 \times 10^{9}$ & $4.4 \times 10^{9}$ & $67 \%$ (LOP) & 74 \\
Sapphire & - & - & $6 \times 10^{7}$ & PL narrowed (1.6 meV) & 77 \\
Sapphire & Migration enhanced & $3.5 \times 10^{9}$ & $2.6 \times 10^{8}$ & Device life time (5000 h) & 69 \\
\hline \hline
\end{tabular}

function of the injection current measured from 50 LEDs at room temperature. The LOP of the UV-LEDs/NPS exhibited higher LOP by $67 \%$ than that of the reference device.

To summarize the ongoing research activities on the ELOG growth scheme, it is an outstanding approach to achieve efficient UV-photonic devices by means of reducing the defects at the growth fronts. These growth fronts were later utilized as templates for the overgrowth of the device structures. Table 2 consolidates the reduction of TDDs in the device structures using the ELOG scheme. Recently, research efforts have been demonstrated to address the problem of large lattice and thermal expansion mismatch by growing $\mathrm{GaN}$ on layered transition metal dichalcogenides (TMDs), e.g., $\mathrm{MoS}^{2}$ and $\mathrm{WeS}^{2}$, with the sapphire substrates beneath. ${ }^{78-81}$ The similar route can be used in growing $\mathrm{AlGaN}$ for the $\mathrm{UV}$ wavelengths in addition to the ELOG scheme.

\subsection{Substrate Patterning}

Various substrate patterning approaches have been suggested to enhance the performance of IIInitrides-based devices grown on sapphire substrates. Among them, the patterned sapphire substrate (PSS), i.e., sapphire substrate surface patterning with nanometer-to-micrometer-sized artificial structures, has been proven to be very promising to enhance the device's performance regarding the IQE and LEE. While the IQE is improved due to the reduction of TDDs by selective-area growth, the LEE is improved due to the photon scattering at the uneven sapphire surface.

A typical patterning process of the sapphire substrate surface involves several steps, as shown in the upper part of Fig. 15. After substrate cleaning, the surface is coated with a resist layer. Then, the desired spatial resolution and shape of the pattern are determined by a designed mask using a relevant lithography method. The patterning process ends with the resist development. During this step, the resist is selectively removed. The second part of the PSS fabrication is to transfer the resist structure, i.e., pattern, to the sapphire substrate. Here, two different ways can be applied as shown in the lower part of Fig. 14; either by an additive technique by depositing a thin film on the substrate surface or using a subtractive technique by etching the substrate surface. Ultimately, the PSS process ends with the resist removal. In the literature, different shapes of PSS have been suggested and fabricated. Figure 16 shows SEM images for some examples of PSS.

Here, we review the use of PSS to enhance the performance of the UV-photonic devices by taking into account the approach used to fabricate the PSS (additive or subtractive techniques). The utilization of PSS for Flip-Chip UV-LED is also discussed. Thereafter, the advantage of using substrate sidewall roughening for UV-LEDs is presented. Ultimately, a few examples of patterning non-sapphire substrates, i.e., Si substrates for UV applications, are discussed. 


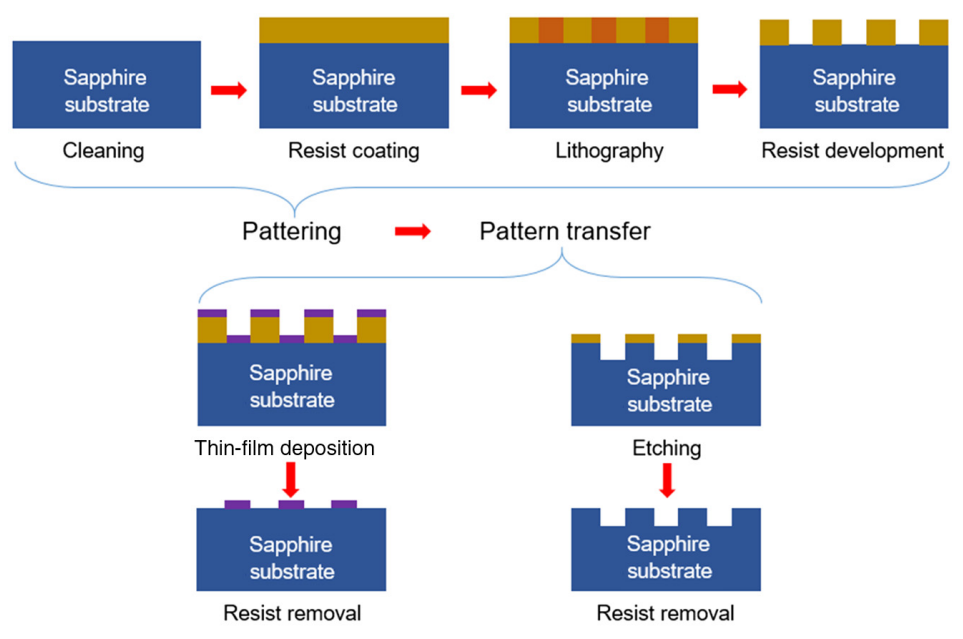

Fig. 15 Typical patterning and pattern transfer process to fabricate a PSS.

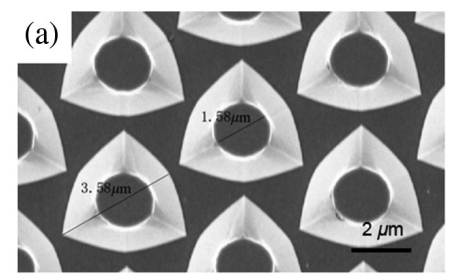

(d)

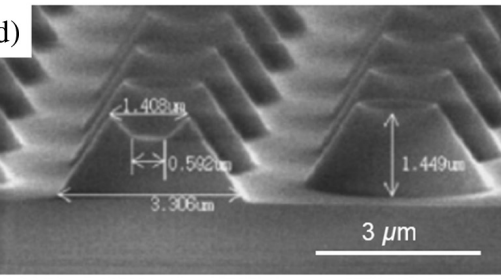

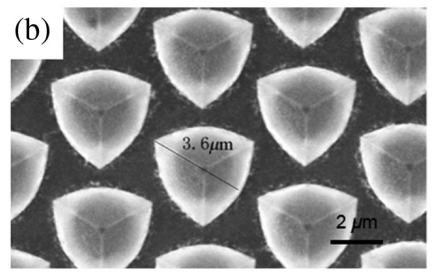

(e)
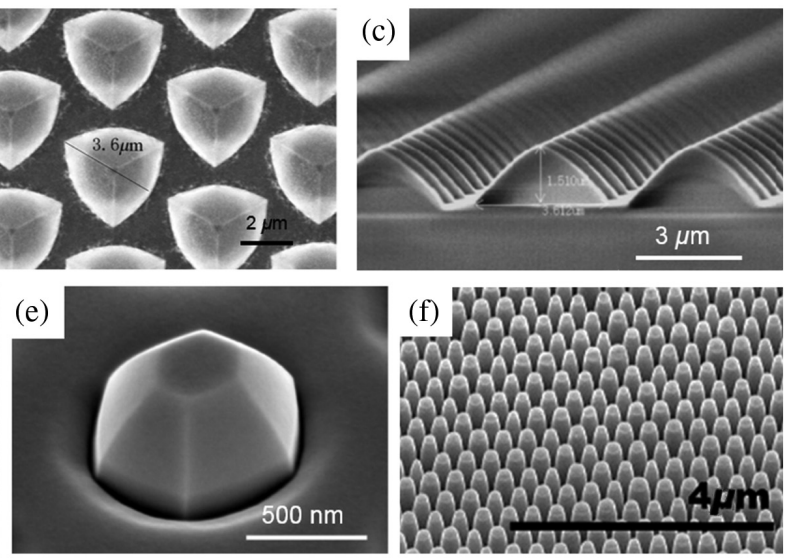

Fig. 16 SEM images show PSS shapes fabricated by subtractive techniques: (a) triangle platform, reproduced with permission from Ref. 82 (b) triangle cone, reproduced with permission from Ref. 82 (c) hemispheric, reproduced with permission from Ref. 83 (d) volcano, reproduced with permission from Ref. 83. SEM images show patterns fabricated on sapphire substrates by additive technique: (e) GaN hexagonal cones, reproduced with permission from Ref. 84 and $(f)$ self-assembled patterned AIN NRs, reproduced with permission from Ref. 73.

Table 3 summarizes the reported achievements acquired from the use of PSSs for UV-photonic devices.

\subsubsection{PSS fabrication for UV applications: additive techniques}

NIL was used to pattern a nanoholes array on a planar AlN/sapphire template. AlGaN/AlN MQW grown on such templates showed a lasing emission at $271.9 \mathrm{~nm}$ with a threshold power density lower by $68 \%$ than that of the same MQW grown on planar AlN/sapphire templates. ${ }^{89}$ This reduction in the threshold power density is attributed to the improvement in the structure quality reflected by the TDDs in the structure grown on nanopatterned AlN/sapphire templates.

Nanoscale patterning in an AlN layer, resulting in self-assembled NRs on a sapphire substrate as shown in Fig. 16(f), was prepared by an inductively coupled plasma (ICP) etching using a selfassembled monolayer of silica spheres on the AlN layer as a lithographic mask. ${ }^{73}$ Growing an AlN layer above these NRs, a reduction in the edge-type dislocation density by approximately two orders of magnitude is demonstrated. Crack-free UV-LEDs emitting at 280-nm wavelength 
Alias et al.: Review of nanophotonics approaches using nanostructures...

Table 3 Summary of achievements acquired from the use of PSSs for UV-photonic devices.

\begin{tabular}{|c|c|c|c|c|c|}
\hline PSS shape & $\begin{array}{l}\text { Lithography } \\
\text { method }\end{array}$ & Device/structure & $\begin{array}{l}\text { Wavelength } \\
(\mathrm{nm})\end{array}$ & $\begin{array}{l}\text { Enhancement/ } \\
\text { achievement }\end{array}$ & Ref. \\
\hline Stripe & Photo- & InGaN/GaN LEDs & 400 (UVA) & $127 \%$ (output power) & 85 \\
\hline Volcano & Photo- & InGaN/GaN LEDs & 380 (UVA) & 2.8 larger LEE & 83 \\
\hline Nano- & Nanosphere & AIGaN-based LEDs & 282 (UVB) & $\begin{array}{l}98 \% \text { (output power) } \\
\text { with } 3.45 \% \text { EQE }\end{array}$ & 86 \\
\hline Microlenses & Photo- & $\begin{array}{c}\text { Flip-chip AIGaN-based } \\
\text { LEDs }\end{array}$ & 280 (UVB) & $55 \%$ (output power) & 87 \\
\hline Nanorods & Nanosphere & AlGaN-based LEDs & 280 (UVB) & $67 \%$ (output power) & 74 \\
\hline Groove & Photo- & AIGaN-based LEDs & 278 (UVC) & $\begin{array}{l}8.4 \mathrm{~mW} \text { output power } \\
\text { with } 3.4 \% \mathrm{EQE}\end{array}$ & 88 \\
\hline Nanoholes & Nanoimprint & $\begin{array}{l}\text { AIGaN/AIN MQW } \\
\text { with lasing emission }\end{array}$ & 271.9 (UVC) & $\begin{array}{l}68 \% \text { reduction in the } \\
\text { threshold power density }\end{array}$ & 89 \\
\hline Groove & Photo- & AIGaN-based LEDs & 266 (UVC) & $\begin{array}{l}5.3 \mathrm{~mW} \text { output power } \\
\text { with } 1.9 \% \mathrm{EQE}\end{array}$ & 88 \\
\hline $\begin{array}{l}\text { Hybrid photonic } \\
\text { nanostructure }\end{array}$ & Nanoimprint & $\begin{array}{c}\text { Flip-chip AIGaN-based } \\
\text { LEDs }\end{array}$ & 265 (UVC) & $\begin{array}{l}\text { About } 20 \text { times } \\
\text { (output power) }\end{array}$ & 90 \\
\hline $\begin{array}{l}\text { Hybrid photonic } \\
\text { nanostructure }\end{array}$ & Electron-beam & $\begin{array}{c}\text { Flip-chip AIGaN-based } \\
\text { LEDs }\end{array}$ & 265 (UVC) & $196 \%$ (output power) & 91 \\
\hline Nanorods & $\begin{array}{l}\text { Lithography- } \\
\text { free }\end{array}$ & $\begin{array}{l}\text { Core-shell-type } \\
\text { AIGaN/AIN MQW }\end{array}$ & 260 (UVC) & 40-times (PL intensity) & 92 \\
\hline
\end{tabular}

have been successfully grown on a 4-in. AlN-NRs/sapphire substrates. ${ }^{74}$ The latter was fabricated by utilizing nanosphere lithography [Fig. 14(a)] and ELOG. The output power of these LEDs is enhanced by $67 \%$. This enhancement is attributed to the formation of embedded periodic air-voids-incorporated nanoscale patterns [Fig. 14(b)], which cause an improvement in the crystal quality of epitaxial layers. Simultaneously, the LEE of this device is enhanced by breaking the predominant in-plane-guided propagation of the photons.

Recently, AIN NRs were prepared on a sapphire substrate by a catalyst/lithography-free method, which is polarity-selective epitaxy and etching. ${ }^{92}$ Core-shell-type AlGaN/AIN MQW emitting at 260-nm wavelength and grown on these AIN NRs show an improvement by 40 times in the PL intensity compared with the same structure grown on planer AlN template.

\subsubsection{PSS fabrication for UV applications: subtractive techniques}

Stripe PSSs have been suggested to improve the light-output efficiency of near-UV-LEDs emitting at around 409 -nm wavelength. ${ }^{93}$ These substrates were fabricated by a standard photolithography method and etched by the ICP etching. The LEDs grown on such a stripe PSS showed an enhancement in the LOP $\sim 20 \%$ over the same LEDs grown on the nonpattern sapphire substrate. In addition to the reduction in TDDs, this enhancement is attributed to the geometric effect of the stripe PSS adding the escape probability of the guided light. Furthermore, Pan et al. studied the effects of both the stripe depth and the stripe orientation on the performance of GaN-dased LEDs emitting at 400-nm wavelength. ${ }^{85}$ They employed a set of PSSs with periodic stripe patterns prepared by photolithography and wet chemical etching. The output power of LEDs is enhanced by $87 \%$ when they were grown on a $0.9-\mu \mathrm{m}$-deep PSS with stripes along $<11$ $20>$ direction. Further an improvement of $40 \%$ in the output power was achieved when the LEDs were grown on a $0.9-\mu \mathrm{m}$-deep PSS with stripes along the $<1-100\rangle$ direction.

Hemispherical- and volcano-shaped PSSs, shown in Figs. 16(c) and 16(d), respectively, were also suggested to enhance the LEE of GaN-based LEDs ${ }^{83}$ The LEE of LEDs emitting at $380 \mathrm{~nm}$ 

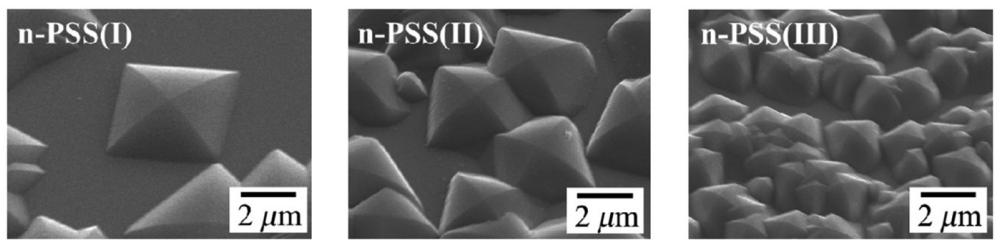

Fig. 17 Pyramidal-shaped PSS substrates prepared without lithography. Reproduced with permission from Ref. 97.

and grown on the optimized volcano-shaped PSS is higher by about 2.8 times than that of the LED grown on the planar sapphire substrate and is enhanced by about 1.6 times in comparison with that of the LED grown on the hemispherical PSS. Zhou et al. ${ }^{94}$ studied the effects of the of PSS size on the performance of UV-LEDs emitting at $375 \mathrm{~nm}$. In this study, they used coneshaped PSS with different sizes that were fabricated by a combination of the thermal-reflow photoresist technique and an ICP etching process. They found that with increasing both the pattern size and PSS fill factor, the TDDs in UV-LEDs decrease. This is due to a reduced $\mathrm{GaN}$ island density that causes fewer grain boundaries and also due to an increased lateral overgrowth area of GaN. Consequently, an improvement in the IQE and LOP of the LED is observed; the output power of the UV-LED grown on a large PSS with a fill-factor (FF) of 0.71 was $131.8 \%$ higher than that of the same LED grown on a small PSS with a FF of 0.4.

In 2013, Dong et al. ${ }^{86}$ fabricated nano-PSSs by nanosphere lithography and have demonstrated the advantage of using these PSSs to enhance the performance of AlGaN-based deep UV-LEDs. In particular, the output power of the UV-LED emitting at 282-nm wavelength of about $3 \mathrm{~mW}$ with $3.45 \% \mathrm{EQE}$ is enhanced by $\sim 98 \%$ compared with that of the UV-LED grown on the nonpatterned substrate. LIL combined with dry-etching and MOCVD has been used to fabricate highly periodic AlN lateral polar structures on a sapphire substrate with submicron domain widths. ${ }^{95}$ Such structures can be used for quasiphase matching to exploit frequency doubling via second harmonic generation in UV-C.

Using a double-sided PSS for GaN-based LEDs, an improvement in the device performance is numerically predicted for different pattern shapes. ${ }^{96}$ Experimentally, nanoscale patterns on a double-sided sapphire substrate were fabricated using NIL and ICP etching. The output power of the LED grown on the double-sided PSS was 52\% greater than the control sample. Furthermore, the use of a double-sided patterned substrate results in an increase in the heat dissipation efficiency and hence a decrease in the LED thermal resistance. Pyramidal-shaped PSS substrates were prepared by a wet-etching without lithography and dry-etching processes ${ }^{97}$ Figure 17 shows the pattern morphology of three substrates used for this study. LEDs grown on these substrates showed an enhancement in the output power by 46.4 to 51.5\% compared with that of the same LEDs grown on flat sapphire substrates. Furthermore, LEDs are grown on the n-PSS(III) substrate (Fig. 17) with 73\% pattern coverage has the highest output power.

\subsubsection{PSS for flip-chip UV-LEDs}

Flip-chip designs have been used for AlGaN-based UV-LEDs because conventional LED contact layer material is absorbing in the UV spectral range. To enhance the LEE of flip-chip UV-LEDs, an approach to integrate monolithic arrays of the microlens to sapphire substrates was suggested.$^{87}$ In particular, UV-LEDs were bonded on high thermal conductive AlN ceramic submounts to improve the thermal dissipation, whereas the emitted UV light was extracted through sapphire substrates with microlenses. The latter were fabricated by photoresist reflow and dryetching. An enhancement by $55 \%$ in the output power at $20 \mathrm{~mA}$ is reported for a UV-LED emitting at 280-nm wavelength with microlenses compared with the same structure fabricated without microlenses. ${ }^{87}$ This enhancement is attributed to the reduced internal reflections of the light caused by the microlens surface profile.

Groove-shaped PSSs were fabricated using photolithography and dry-etching. A high-quality lateral epitaxy overgrowth-AIN templates grown on these PSSs have enabled to realize flipchip AlGaN-based UV-LEDs that are emitting at 266- and 278-nm wavelengths with an output 

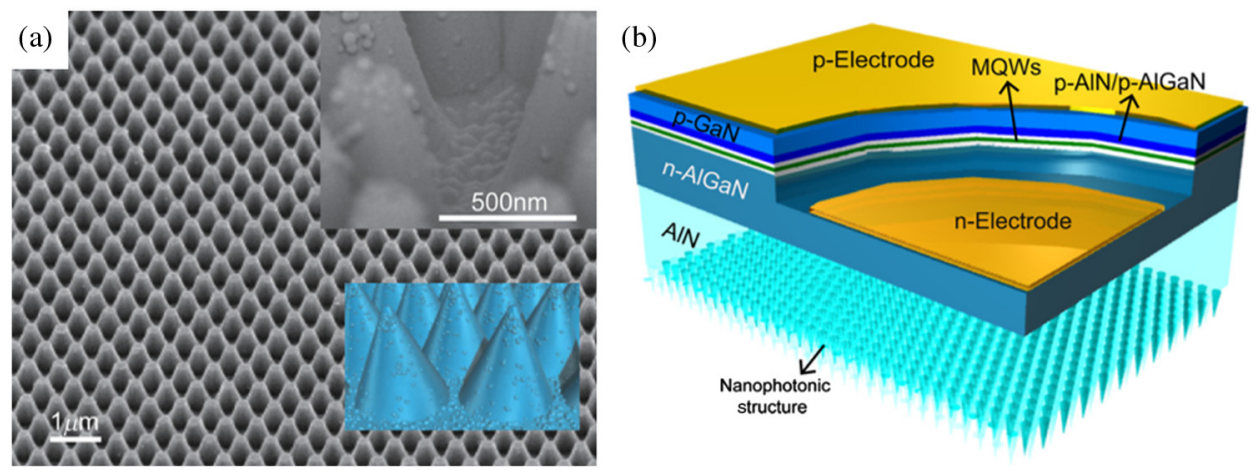

Fig. 18 (a) SEM image of fabricated light extraction structures: a triangular lattice of circular AIN cones with subwavelength nanostructures. The insets show an enlarged SEM image and a schematic. (b) Schematic of UV-LED grown on an AIN substrate with nanophotonic light extraction structures. Reproduced with permission from Ref. 91.

power of $5.3 \mathrm{~mW}$ with $1.9 \% \mathrm{EQE}$ and $8.4 \mathrm{~mW}$ with $3.4 \% \mathrm{EQE}$, respectively ${ }^{88}$ Flip-chip UVLEDs on AlN substrates show a relatively low light extraction and low output power. This is due to the total internal reflection (Fresnel reflection) at the interface between the AlN surface and the ambient medium and to the internal optical absorption by the $p-\mathrm{GaN}$ contact layer and the bulk AlN substrate. To overcome the problem of the Fresnel reflection, the AlN bottom-side surface was constructed to have a configuration composed of a hybrid structure of $\mathrm{PhCs}$ and subwavelength nanostructures. This hybrid structure is shown in Fig. 18(a) and was realized using EBL and subsequent ICP etching. ${ }^{91}$ Growing a UV-LED emitting at $265 \mathrm{~nm}$ on the resulting AlN substrate consequently led to an enhancement in the LED output power by $196 \%$ (the output power of $90 \mathrm{~mW}$ at $350 \mathrm{~mA}$ ). Figure 18(b) shows a schematic drawing of the UV-LED used for this study.

Despite the advantage of this relatively high output power achieved in the aforementioned study, ${ }^{91}$ the employed fabrication method, i.e., EBL, is time-consuming, and hence this limits the patterned device size. To overcome this issue, a large-area AlN nanophotonic light-extraction structure fabricated by NIL is used to realize UV-LEDs emitting at $265 \mathrm{~nm} .{ }^{90}$ Furthermore, an improved design of a multiple-strip $p$-electrode geometry for these LEDs results in a uniform current density distribution. Consequently, an output power higher than $150 \mathrm{~mW}$ has been recorded with low-efficiency droop. Also, an increase by $\sim 20$ times in the output power over that of a conventional flat-surface LED was achieved.

\subsubsection{Substrate sidewall roughening}

Substrate sidewall roughening is an approach suggested to enhance the LEE of AlGaN-based UV-LEDs. ${ }^{98}$ This approach has revealed that a roughening region for effective light extraction enhancement exists on the substrate's sidewall. Roughening outside the effective roughening region will degenerate the sidewall light extraction due to inward photon scattering and absorption. Picosecond laser dicing was used to form roughening layers on the substrate's sidewalls without introducing thermal damage. A UV-LEDs emitting at 275-nm wavelength with three roughening layers shows $13.2 \%$ higher output power than the same LEDs with two roughening layers. ${ }^{98}$

\subsubsection{Patterning non-sapphire substrate for UV applications}

A UV-LED emitting at 359-nm wavelength was fabricated on a stripe-patterned Si substrate. ${ }^{99}$ To achieve this, a fully coalesced ELOG AlN layer with a 7- $\mu$ m thickness was used as a template for the UV-LED growth. This LED was fabricated in back-emission configuration, and the Si substrate was removed after the flip-chip bonding. An enhancement in the output light extraction by $50 \%$ is observed for the back-emission LED compared with the top emission one. Likewise, a high crystal quality AlN template was used to grow an AlGaN-based UV-LED on a microcircle- 
patterned Si substrate. ${ }^{100}$ The latter was achieved using standard lithography and ICP etching. The fabricated UV-LED emits at $325 \mathrm{~nm}$ with an EQE of about $0.03 \%$.

Cantilever epitaxy method was used to grow AlN layers on 200-nm-nanopatterned Si substrate. ${ }^{101}$ This substrate was patterned into nanostripes with nominally $150-\mathrm{nm}$ wide ridges and 50-nm wide trenches $\mathrm{Si}$, with an etching depth of $100 \mathrm{~nm}$ using EBL and dry-etching. The resultant AlN/Si structures were used as templates to grow UV-LEDs emitting at 344-nm wavelength with a peak pulse power of about $0.7 \mathrm{~mW}$.

\subsection{Surface Roughening}

Surface roughening has been proven to be an effective approach to enhance the LEE of UV-LEDs. Next, we grouped the surface roughening into surface texturing and surface patterning.

\subsubsection{Surface texturing}

Surface texturing architectures help to improve the surface emission area and increase the probability of photons to escape out of the surface by the angular randomization. ${ }^{102}$ Lee et al. ${ }^{102}$ fabricated a UV-LED device emitting at $365 \mathrm{~nm}$ and obtained a total enhancement by a factor of 7.8 compared with the conventional UV-LED. This is attributed to the surface structuring, which exhibits random scattering effects. Figure 19(a) shows the schematic of the devices showing the roughening surface with root-mean-square (rms) roughness of $\sim 398 \mathrm{~nm}$ by immersing the sample in $45 \mathrm{wt} . \% \mathrm{KOH}$ at $110^{\circ} \mathrm{C}$ for $180 \mathrm{~s} .{ }^{102}$ Figure 19 (b) shows the L-I-V characteristics of three samples emphasizing the enhancement in the overall performance toward surface-roughened LED. Furthermore, Chiang et al. ${ }^{103}$ achieved an enhancement in the output power by $525 \%$ and in the EQE by 6.5 times for a UVLED emitting at $365 \mathrm{~nm}$, compared with a conventional LED, by a combination of the noninsulation current blocking layer, wafer bonding, laser lift-off, and surface treatment processes. Figures 19(d) and 19(e) show a schematic drawing of the fabrication steps and SEM images for the roughened surface, respectively. A high improvement in the L-I-V characterization was realized as shown in Fig. 19(e).

Inoue et al. ${ }^{90}$ reported a high output power of $150 \mathrm{~mW}$ of a UV-LED operating at $265 \mathrm{~nm}$. The UV-LED structure was fabricated on AlN substrate to minimize the lattice and thermal expansion mismatches between the substrate and the epitaxial layers. However, the high refractive index (RI) of the AlN substrate results in a small light escape cone angle. Imprinted polymer patterns by NIL were performed to create a large-area AIN nanophotonic structure, which was capable of reaching an improved EQE by $\sim 4$ times compared with a flat LED, as shown in Figs. 19(f)19(h). Chiang et al. ${ }^{104}$ have suggested an abraded surface structuring method for a thin-GaN (NTG) UV-LED as shown in Fig. 19(i). As a result, the LED emitting at $365 \mathrm{~nm}$ shows an output power enhancement of $\sim 18.3 \%$ to $27.4 \%$ and EQEs of $20 \%$ to $27.2 \%$ at an injected current of 700 and $1500 \mathrm{~mA}$, respectively. The enhancements in the output power and the EQE were attributed to the elimination of the electrode effect due to a micrometer-range abraded surface modification after an etching process using $\mathrm{KOH}$ solution. Additionally, a 2.5-times light extraction gain has been reported by Zhou et al. ${ }^{105}$ by utilizing a roughening process using photoelectrochemical etching. As a result, an enhancement output power of $\sim 4.6$ and $\sim 2.5$ times was achieved for 280- and 325-nm emitting LEDs, respectively.

Khizar et al. ${ }^{87}$ have reported an enhancement by $55 \%$ in the output power of the UV-LED emitting at $280 \mathrm{~nm}$ by integrating monolithic microlens arrays. This is due to curvature surface profile of the microlenses, which helps in increasing light reflections, in which more light can be extracted and as a result, LEE is enhanced. In a subsequent report, Kim et al. ${ }^{106}$ demonstrated an enhanced LEE by $23 \%$ for UV-LED emitting at by utilizing $\mathrm{SiO}_{2}$ nanosphere arrays. The nanosphere arrays act like nanolenses with a hexagonal-like pattern when immersed into a BCB resin and undergo a heat treatment. The hemispherical pattern leads to the random direction of the light rays with the LEE increased by multiscattering effects. Recently, Liu at al. ${ }^{107}$ characterized a UV-LED that operates at $214 \mathrm{~nm}$ via nanowalls structures and calculated an IQE of $60 \%$. Alternative nanostructures features have been detailed by others, e.g., large-scale nanostructure nanosphere, ${ }^{108}$ silica glass with tailored nanostructures, ${ }^{109}$ and truncated cones. ${ }^{110}$ 

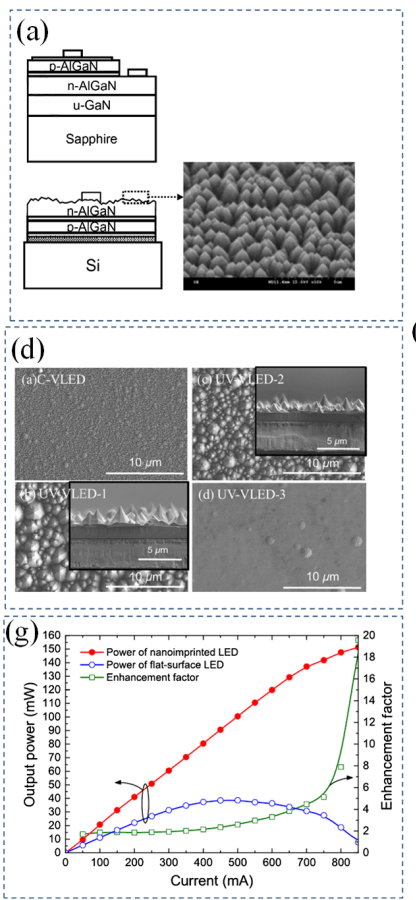

(b)

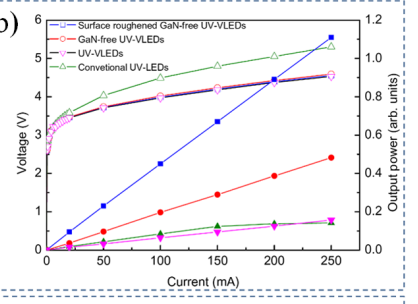

(e)

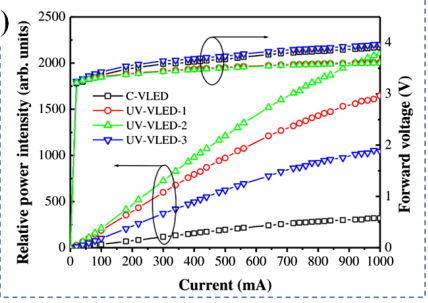

(h)

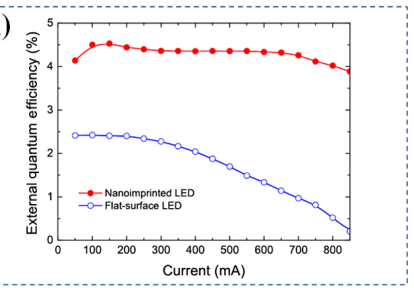

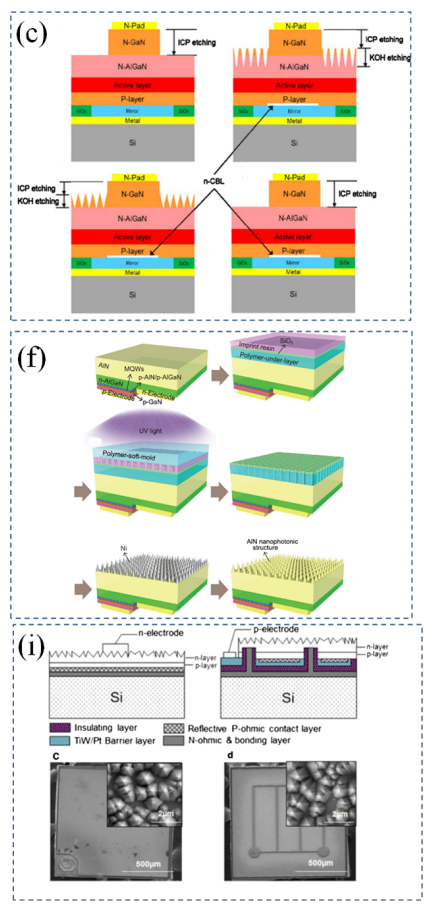

Fig. 19 (a) Schematic of the conventional LED (top) and the roughened LED (bottom) with SEM image (inset). Reproduced with permission from Ref. 102. (b) L-I-V characteristics of the samples. Reproduced with permission from Ref. 102. (c) Schematic of the fabrication steps for the UV-LED. Reproduced with permission from Ref. 103. (d) SEM images under different etching conditions. Reproduced with permission from Ref. 103. (e) L-I-V characteristics of UV-LEDs. Reproduced with permission from Ref. 103. (f) Schematic of the fabrication steps for the nanophotonic light-extraction structure. Reproduced with permission from Ref. 90. (g) Output power and enhancement factor. Reproduced with permission from Ref. 90. (h) EQE as a function of the current. Reproduced with permission from Ref. 90. (i) Schematic and SEM images of devices with the micro abraded surface (insets). Reproduced with permission from Ref. 104.

\subsubsection{Surface patterning}

Recently, a light-extraction mechanism based on an Rh mirror electrode and a transparent p-type AlGaN:Mg contact layer has been investigated by Takano et al. ${ }^{111}$ They successfully achieved a $20 \%$ enhancement in EQE at an emission wavelength of $275 \mathrm{~nm}$. In this work [Fig. 20(a)], a conventional AlGaN LED is modified into a UV-LED structure by utilizing the following features: (1) crack-free AlN on the patterned sapphire substrate, (2) AlGaN:Mg p-type contact layer, (3) mirror electrode, and (4) Si encapsulation resin. The encapsulation resin helped to reduce the optical loss and reached 89\% transmission. Implementing Rh mirrors into the LED structure results in an improvement in output power from 3.9 to $18.3 \mathrm{~mW}$ at $20 \mathrm{~mA}$ and in the EQE from $4.3 \%$ to $20.3 \%$ in comparison with the conventional LEDs. Increased multireflection of the light in the chip and absorption reduction helps to improve the device performance. Figure 20(b) shows the device characteristics (output power and EQE). The encapsulated LED in hemispherical resin on a Si submount is shown in Fig. 20(c). Encapsulation using other UV-transparent material, e.g., AlN-doped fluoropolymer and BVE polymer with $-\mathrm{CF}_{3}$ ends, has been demonstrated recently. ${ }^{113,114}$ Additionally, several research groups demonstrated pixel-LEDs (by meshing the $p$-contact metals) to enhance the output power and EQE characteristics. ${ }^{115,116}$ However, the pixels were fabricated at the microscale and introduced complexity into the LED fabrication.

A $70 \%$ improvement in output power at peak emission of $276 \mathrm{~nm}$ has been reported by Fayisa et al. by employing an inclined sidewall at the $\mathrm{p}-\mathrm{GaN}$ with $\mathrm{MgF}_{2} / \mathrm{Al}$ omnidirectional reflectors. ${ }^{112}$ Figure 20(d) shows the schematic fabrication process of AlGaN-based UVLEDs with a $5 \times 5$ array of the microring active mesa. The microring array consists of a truncated 


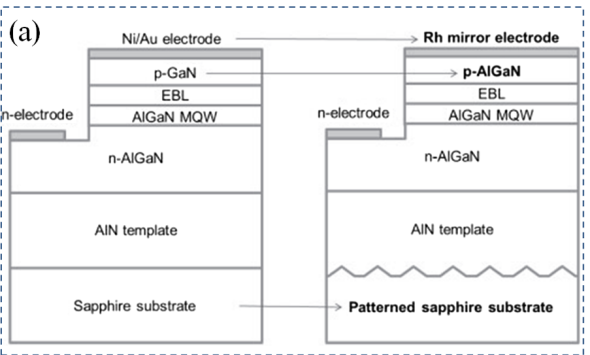

(d)

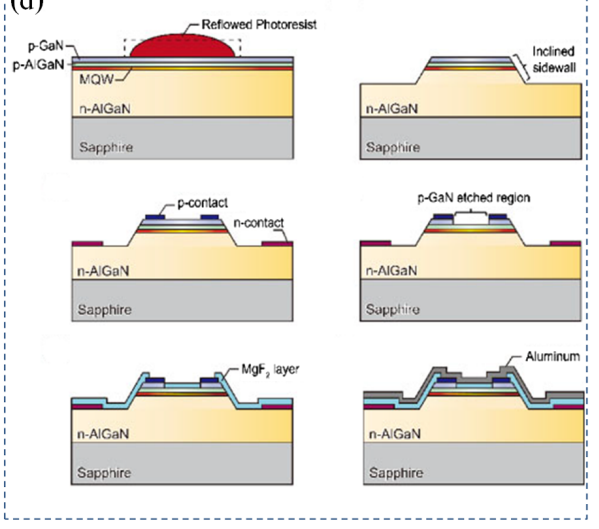

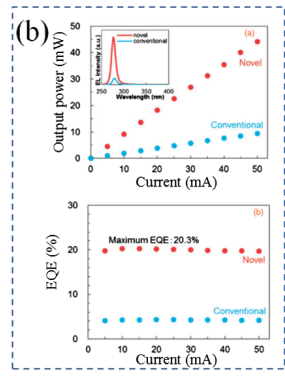

(c)

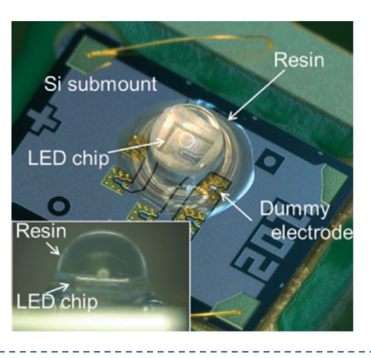

(e)

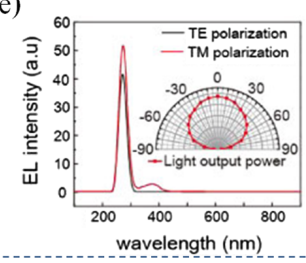

(f)
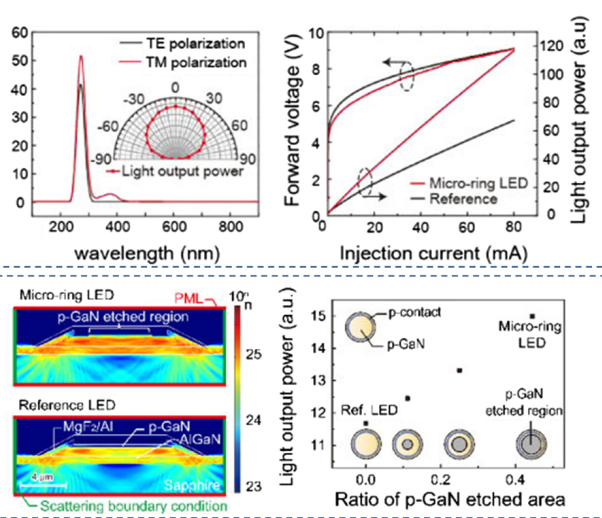

Injection current (mA)

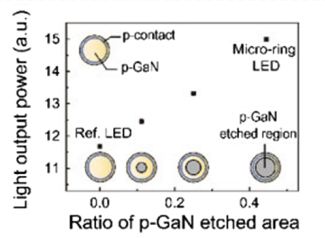

Fig. 20 (a) Schematics of conventional (left) and UV-LEDs (right). Reproduced with permission from Ref. 111. (b) Output power (top) and EQE (bottom) characteristics. Reproduced with permission from Ref. 111. (c) Encapsulated UV-LED in hemispherical resin. Reproduced with permission from Ref. 111. (d) Schematic of fabrication steps for UV-LED with micro-ring active mesa. Reproduced with permission from Ref. 112. (e) EL spectra of TE and TM polarized light with the angle-dependent output power profile (inset) and L-I-V curve of the devices. Reproduced with permission from Ref. 112. (f) Electrical field distribution of the devices (left) and the calculated output power of the devices as a function of the etched area to mesa ratio (right). Reproduced with permission from Ref. 112.

cone-shaped active mesa, which is covered by the inclined $\mathrm{MgF}_{2}$ sidewall of Ti/Al/Ni/Au ohmic contacts. The $p$-GaN-layer etching was performed by ICP etching to reflect photons down to the substrate without being absorbed. Decreasing the light absorption and directing strong sidewallheading TM polarized light via the $\mathrm{MgF}_{2} / \mathrm{Al}$ reflectors leads to significant enhancement in the LEE and output power as shown in Figs. 20(e) and 20(f), respectively.

Table 4 summarizes the recent applications of surface roughening to enhance the performance of UV-LEDs. In this table, the device characteristics are described regarding initial emission wavelength $\left(\lambda_{o}\right)$, initial threshold current $\left(I_{o}\right)$, and initial output power $\left(P_{o}\right)$.

\subsection{Photonic Crystals}

PhCs are inhomogeneous dielectric structures with periodic modulation of the RI, which allows the control and manipulation of the light propagation. PhCs can form a photonic bandgap where the light cannot propagate through the structure within a range of frequencies. Concerning the geometry of the structure, $\mathrm{PhCs}$ can be divided into one-, two-, and three-dimensional (1-D, 2-D, and 3-D) PhCs. In 1-D PhCs, the periodic modulation of RI occurs in one direction only, whereas RI stays uniform in the other two directions. 1-D PhCs have been widely used for several photonic devices, e.g., LDs, LEDs, and PDs in the form of a distributed Bragg reflector (DBR) as a highly reflective mirror. The most important aspect of the 2-D/3-D PhCs design is the lattice pattern choice. Most widely studied two-dimensional (2-D) PhCs either are with a hexagonal (triangular) or square lattices. In general, 1-D PhCs can be formed using thin-films deposition at various growth temperatures to suit the material or process requirements. ${ }^{117-123}$ As for the 2-D/3$\mathrm{D} \mathrm{PhCs}$, it can be fabricated using EBL, photolithography, NIL, or direct laser writing, followed by various etching techniques to form the air holes or pillars of a layer. ${ }^{124-128}$ 
Alias et al.: Review of nanophotonics approaches using nanostructures...

Table 4 Recent progress of using surface roughening approach for UV-LEDs improvements.

\begin{tabular}{|c|c|c|c|c|c|c|}
\hline \multirow[b]{2}{*}{ Technique } & \multirow[b]{2}{*}{ Device } & \multicolumn{3}{|c|}{ Device characteristic } & \multirow[b]{2}{*}{ Enhancement } & \multirow[b]{2}{*}{ Ref. } \\
\hline & & $\lambda_{0}(\mathrm{~nm})$ & $I_{o}(\mathrm{~mA})$ & $P_{o}(\mathrm{~mW})$ & & \\
\hline \multirow[t]{5}{*}{ Surface structuring } & UVC-LED & 265 & 850 & 150 & 20-fold $\left(P_{o}\right)$ & 90 \\
\hline & UVC-LED & 280 & NA & NA & $2.5 \times($ LEE $)$ & 105 \\
\hline & UVA-LED & 365 & 350 & 858 & $6.5 \times$ EQE and $525 \%$ in $P_{o}$ & 103 \\
\hline & UVA-LED & 365 & 250 & NA & $7.8 \times\left(P_{o}\right)$ & 102 \\
\hline & UVA-LED & 365 & 1500 & 700 & $\begin{array}{c}20 \% \text { in EQE and } \\
18.3 \%\left(P_{o}\right)\end{array}$ & 104 \\
\hline \multirow{8}{*}{$\begin{array}{l}\text { Nanostructures } \\
\text { features }\end{array}$} & UVC-LED & 214 & 170 & NA & $60 \%$ (EQE) & 107 \\
\hline & UVC-LED & 250 & 50 & 7.89 & $8.6 \%$ in light emission & 109 \\
\hline & UVC-LED & 275 & 20 & 11.6 & $20 \%$ (EQE) & 111 \\
\hline & UVC-LED & 280 & 200 & 472 & $70 \%\left(P_{o}\right)$ & 112 \\
\hline & UVC-LED & 280 & 20 & $0.2-0.3$ & $55 \%\left(P_{o}\right)$ & 87 \\
\hline & UVC-LED & 280 & 100 & NA & $37 \%$ in light emission & 110 \\
\hline & UVA-LED & 375 to 400 & NA & NA & $23 \%(P L)$ & 106 \\
\hline & UVA-LED & 370 & $\mathrm{Na}$ & NA & $\begin{array}{l}2.5 \text { fold in band } \\
\text { edge luminescence }\end{array}$ & 108 \\
\hline \multirow[t]{2}{*}{ Encapsulates } & UVC-LED & 280 & 200 & 472 & $1.5 \times(\mathrm{LEE})$ & 114 \\
\hline & UVC-LED & 280 & 120 & NA & $16.4 \%\left(P_{o}\right)$ & 113 \\
\hline
\end{tabular}

\subsubsection{1-D Photonic crystals: distributed Bragg reflector}

A DBR is formed by multiple stacking of two alternate high and low RI layers with the thickness of each layer equivalent to the quarter-wave of the designated wavelength. DBRs have been used as high reflective mirrors in many UV-based photonic devices (summarized in Table 5), e.g., vertical-cavity surface-emitting laser (VCSEL), ${ }^{129-131}$ edge-emitting laser (EEL) ${ }^{139}$ microdisk LD,${ }^{137}$ resonant-cavity LED (RCLED) ${ }^{132}$ resonant-cavity PD (RCPD), ${ }^{133}$ and LEDs. ${ }^{70,134-136,138}$ These DBRs were formed using oxides named as UV-dielectric DBR and group III-nitride semiconductors, named as UV-nitride DBR. Most of the aforementioned UVbased photonic devices require DBR(s) with high reflectivity, $R>99 \%$. To achieve such a high $R$, the contrast in RI must be high to have a smaller period and broad stopband, the chosen materials exhibit a high transparency at the device operating wavelength, the material interfaces must be abrupt with smooth surface to reduce the optical loss, and the chosen materials are compatible with lattice-matching to subsequent material deposition/growth.

Compared with UV-nitride DBRs, UV-dielectric DBRs offer more extensive material choices (no lattice-matching limitation) and flexibility in the material growth or deposition technique, e.g., sputtering, PECVD, and electron beam evaporation. Importantly, highly reflective DBR $(R>99 \%)$ and broad stopband $(>30 \mathrm{~nm})$ can be easily obtained within a small number of repetitions (typically 10 to 15 periods) due to the high RI contrast among oxide materials. Table 6 lists some examples of oxides-based DBRs that have been demonstrated in the UV spectral range, e.g., $\mathrm{ZrO}_{2} / \mathrm{SiO}_{2},{ }^{140} \mathrm{Ta}_{2} \mathrm{O}_{5} / \mathrm{SiO}_{2},{ }^{129} \mathrm{TiO}_{2} / \mathrm{SiO}_{2},{ }^{130}$ and $\mathrm{Si}_{3} \mathrm{~N}_{4} / \mathrm{SiO}_{2}{ }^{141}$ for UV-A; $\mathrm{Al}_{2} \mathrm{O}_{3} / \mathrm{SiO}_{2}{ }^{142}$ for UV-B; $\mathrm{HfO}_{2} / \mathrm{SiO}_{2}{ }^{143}$ and $\mathrm{YDH} / \mathrm{SiO}_{2}{ }^{144}$ for UV-C, respectively.

$\mathrm{SiO}_{2}$ is commonly used as the low RI layer in UV-dielectric DBRs as it offers the lowest RI among the dielectrics and has wider energy bandgap. However, most of the materials used for high RI layer in the UV-A DBRs are not transparent at the UV-B and UV-C spectral ranges. Although $\mathrm{Al}_{2} \mathrm{O}_{3}$ is transparent for extensive range till vacuum-UV, ${ }^{142}$ a high number of period 
Alias et al.: Review of nanophotonics approaches using nanostructures...

Table 5 UV-dielectric DBRs and nitride DBRs employed in various photonic devices operating in the UV regime.

\begin{tabular}{|c|c|c|c|c|c|}
\hline Technique & Device & $\begin{array}{l}\text { Device structure } \\
\text { (top-down) }\end{array}$ & Device characteristic & Enhancement & Ref. \\
\hline \multirow[t]{5}{*}{$\begin{array}{l}\text { Top and } \\
\text { bottom } \\
\text { DBRs }\end{array}$} & $\begin{array}{l}\text { UV-LD } \\
\text { (VCSEL) }\end{array}$ & $\begin{array}{l}13.5 \times \mathrm{Ta}_{2} \mathrm{O}_{5} / \mathrm{SiO}_{2} \\
\text { InGaN/GaN MQW } \\
13.5 \times \mathrm{Ta}_{2} \mathrm{O}_{5} / \mathrm{SiO}_{2}\end{array}$ & $\begin{array}{l}\text { Lasing at } 397 \mathrm{~nm} \\
\text { (optical pumping) } \\
\text { and } 65 \% \text { polarization }\end{array}$ & $\begin{array}{l}\mathrm{PL} \text { narrowed } \\
(0.13 \mathrm{~nm})\end{array}$ & 129 \\
\hline & $\begin{array}{l}\text { UV-LD } \\
\text { (VCSEL) }\end{array}$ & $\begin{array}{c}6 \times \mathrm{TiO}_{2} / \mathrm{SiO}_{2} \\
\ln \mathrm{GaN} / \mathrm{GaN} \mathrm{MQW} \\
35 \times \mathrm{GaN} / \mathrm{Al}_{0.34} \mathrm{Ga}_{0.66} \mathrm{~N}\end{array}$ & $\begin{array}{l}\text { Lasing at } 381 \mathrm{~nm} \\
\text { (optical pumping) }\end{array}$ & $\begin{array}{l}\text { PL narrowed } \\
\text { (8 to }<0.1 \mathrm{~nm})\end{array}$ & 130 \\
\hline & $\begin{array}{l}\text { UV-LD } \\
\text { (VCSEL) }\end{array}$ & $\begin{array}{c}7 \times \mathrm{HfO}_{2} / \mathrm{SiO}_{2} \\
\operatorname{InGaN} / \mathrm{AlGaN} \mathrm{MQW} \mathrm{MQW} \\
40.5 \times \mathrm{GaN} / \mathrm{Al}_{0.12} \mathrm{Ga}_{0.88} \mathrm{~N}\end{array}$ & $\begin{array}{l}\text { Lasing at } 374.9 \mathrm{~nm} \\
\text { (optical pumping) }\end{array}$ & $\begin{array}{l}\text { PL narrowed } \\
(0.66 \mathrm{~nm})\end{array}$ & 131 \\
\hline & $\begin{array}{l}\text { UV-LED } \\
\text { (RCLED) }\end{array}$ & $\begin{array}{c}2.5 \times \mathrm{ZrO}_{2} / \mathrm{SiO}_{2} \\
\operatorname{lnGaN} / \mathrm{GaN} \mathrm{MQW}^{\mathrm{MQN}} \\
20 \times \mathrm{GaN} / \mathrm{Al}_{0.27} \mathrm{Ga}_{0.73} \mathrm{~N}\end{array}$ & $\begin{array}{c}\mathrm{EL} \text { at } 390 \mathrm{~nm} \text { and } \\
\text { EQE of } 1.2 \%\end{array}$ & - & 132 \\
\hline & $\begin{array}{l}\text { UV-PD } \\
\text { (RCPD) }\end{array}$ & $\begin{array}{c}3 \times \mathrm{Al}_{0.3} \mathrm{Ga}_{0.7} \mathrm{~N} / \mathrm{AIN} \\
\mathrm{p}-\mathrm{GaN} / \mathrm{i}-\mathrm{GaN}_{\mathrm{n}} \mathrm{n}-\mathrm{Al}_{0.3} \mathrm{Ga}_{0.7} \mathrm{~N} \\
20.5 \times \mathrm{Al}_{0.3} \mathrm{Ga}_{0.7} \mathrm{~N} / \mathrm{AIN}\end{array}$ & $\begin{array}{l}\text { Response at } 330 \mathrm{~nm} \\
\text { and EQE of } 48 \%\end{array}$ & - & 133 \\
\hline \multirow[t]{4}{*}{$\begin{array}{l}\text { Bottom } \\
\text { DBR }\end{array}$} & UV-LED & $\begin{array}{l}\text { InGaN/GaN MQW } \\
9.5 \times \mathrm{HfO}_{2} / \mathrm{SiO}_{2}\end{array}$ & $\mathrm{EL}$ at $390 \mathrm{~nm}$ & $\begin{array}{c}36 \% \text { (TDDs) } \\
75 \% \text { (output power) }\end{array}$ & 70 \\
\hline & UV-LED & $\begin{array}{c}\text { AllnGaN/AIGaN MQW } \\
16 \times \mathrm{Ta}_{2} \mathrm{O}_{5} / \mathrm{SiO}_{2}\end{array}$ & $\mathrm{EL}$ at $365 \mathrm{~nm}$ & $\begin{array}{l}25 \% \text { (output power, } \\
\text { EQE, and LEE) }\end{array}$ & 134 \\
\hline & UV-LED & $\begin{array}{c}\mathrm{GaN} / \mathrm{AIGaN} M Q W \\
12 \times \mathrm{Al}_{0.085} \mathrm{Ga}_{0.915} \mathrm{~N} / \text { air }\end{array}$ & $\begin{array}{l}\mathrm{PL} \text { at } 359.7 \mathrm{~nm} \text { with } \\
\text { linewidth of } 11.43 \mathrm{~nm} \\
\text { (optical pumping) }\end{array}$ & $\begin{array}{c}\mathrm{PL} \text { at } 365.2 \mathrm{~nm} \text { with } \\
\text { linewidth narrowed } \\
(1.18 \mathrm{~nm})\end{array}$ & 135 \\
\hline & UV-LED & $\begin{array}{c}\text { AllnGaN/AllnGaN MQW } \\
25 \times \mathrm{Al}_{0.16} \mathrm{Ga}_{0.84} \mathrm{~N} / \mathrm{Al}_{0.49} \mathrm{Ga}_{0.51} \mathrm{~N}\end{array}$ & $\mathrm{EL}$ at $350 \mathrm{~nm}$ & $\begin{array}{l}2.3 \times(E L \text { and output } \\
\text { power) }\end{array}$ & 136 \\
\hline \multirow[t]{2}{*}{ Top DBR } & $\begin{array}{l}\text { UV-LD } \\
\text { (microdisk) }\end{array}$ & $\begin{array}{l}25 \times \mathrm{Al}_{0.2} \mathrm{Ga}_{0.8} \mathrm{~N} / \mathrm{AIN} \\
\mathrm{GaN} \text { microdisk }\end{array}$ & $\begin{array}{l}\text { Lasing at } 377 \text { to } \\
379 \mathrm{~nm} \text { (optical } \\
\text { pumping) }\end{array}$ & - & 137 \\
\hline & $\begin{array}{l}\text { UV-LED } \\
\text { (flip-chip) }\end{array}$ & $\begin{array}{c}14 \times \mathrm{Ta}_{2} \mathrm{O}_{5} / \mathrm{SiO}_{2} \\
\text { AIGaN/AIGaN MQW }\end{array}$ & $\mathrm{EL}$ at $353 \mathrm{~nm}$ & $25 \%$ (output power) & 138 \\
\hline Side DBR & $\begin{array}{l}\text { UV-LD } \\
\text { (EEL) }\end{array}$ & $\begin{array}{c}5 \times \text { and } 6 \times \mathrm{HfO}_{2} / \mathrm{SiO}_{2} \\
\text { AIGaN/AIGaN MQW }\end{array}$ & $\begin{array}{l}\text { Lasing at } 249 \mathrm{~nm} \\
\text { (optical pumping) }\end{array}$ & $\begin{array}{l}28 \% \text { (threshold } \\
\text { pumping power) }\end{array}$ & 139 \\
\hline
\end{tabular}

is required ( $>40$ periods) and the DBR stopband is not so broad due to insufficient RI contrast. Replacing $\mathrm{Al}_{2} \mathrm{O}_{3}$ by $\mathrm{HfO}_{2}$ as the high RI layer for the DBR is interesting because of high RI contrast. However, $\mathrm{HfO}_{2}$ is absorbing for wavelengths below $250 \mathrm{~nm}$. Recently, a highly reflective UV-C dielectric DBR has been demonstrated using a $\mathrm{YDH} / \mathrm{SiO}_{2}$ material system. ${ }^{144}$ Figure 21(a) shows that YDH thin films are less absorbing compared with $\mathrm{HfO}_{2}$ thin films across the UV wavelengths. In Fig. 21(b), the YDH thin films exhibit wider energy bandgap of $\sim 6.21 \mathrm{eV}$ compared with the $\mathrm{HfO}_{2}$ thin films as measured using a phase-modulated spectroscopic ellipsometry. ${ }^{145}$ This increase in energy bandgap indicates the transparency of the YDH material in the UV-C wavelengths especially below $250 \mathrm{~nm}$. Figure 21(c) shows the reflectivity spectrum of 15 periods $\mathrm{YDH} / \mathrm{SiO}_{2} \mathrm{DBR}$ and the transmission spectrum of single-layer YDH thin films as a function of the wavelength. The reflectivity peak of $R \sim 99.9 \%$ of the DBR is obtained before the absorption from YDH becomes dominant. The achieved stopband of $\sim 50 \mathrm{~nm}$ is quite extensive within the UV-C regime. The inset of Fig. 21(c) shows the crosssectional SEM micrograph of the DBR structure indicating a uniform period thickness distribution with minor roughness introduced. 
Alias et al.: Review of nanophotonics approaches using nanostructures...

Table 6 Examples of UV-dielectric DBRs demonstrated in UV wavelengths.

\begin{tabular}{lcccccc}
\hline \hline $\begin{array}{l}\text { Material } \\
\text { system }\end{array}$ & $\begin{array}{c}\text { Number of } \\
\text { period }\end{array}$ & $\begin{array}{c}\text { Reflectivity } \\
(\%)\end{array}$ & $\begin{array}{c}\text { Center } \\
\text { wavelength } \\
(\mathrm{nm})\end{array}$ & $\begin{array}{c}\text { Stopband } \\
(\mathrm{nm})\end{array}$ & Remark & Ref. \\
\hline $\mathrm{ZrO}_{2} / \mathrm{SiO}_{2}$ & 15 & 99.5 & 399 (UVA) & - & Top mirror InGaN VCSEL & 140 \\
$\mathrm{Ta}_{2} \mathrm{O}_{5} / \mathrm{SiO}_{2}$ & 13.5 & 99.2 & 396 (UVA) & - & Top and bottom mirrors InGaN VCSEL 129 \\
$\mathrm{TiO}_{2} / \mathrm{SiO}_{2}$ & 6 & 97.0 & 381 (UVA) & - & Top mirror InGaN VCSEL & 130 \\
$\mathrm{Si}_{3} \mathrm{~N}_{4} / \mathrm{SiO}_{2}$ & 9 & 95.0 & 355 (UVA) & 80 & Top mirror GaN microcavity & 141 \\
$\mathrm{Al}_{2} \mathrm{O}_{3} / \mathrm{SiO}_{2}$ & 42.5 & 99.0 & 296 (UVB) & 26 to 39 & Mirror only & 142 \\
$\mathrm{HfO}_{2} / \mathrm{SiO}_{2}$ & 11.5 & 250 & 250 (UVC) & 45 & Mirror only & 143 \\
$\mathrm{YDH} \mathrm{SiO}_{2}$ & 15 & 99.9 & 240 (UVC) & 50 & Mirror only & 144 \\
\hline \hline
\end{tabular}

(a)

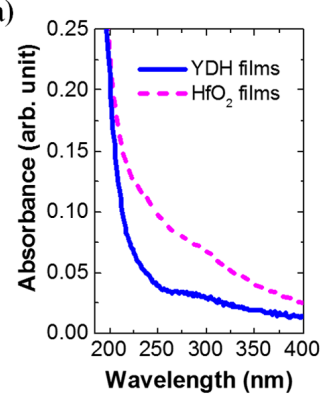

(b)

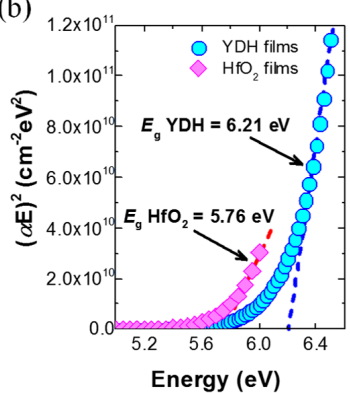

(c)

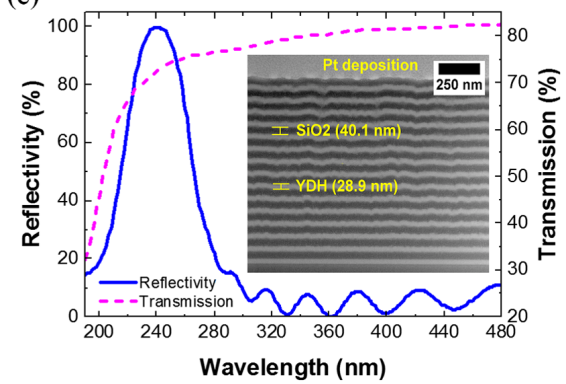

Fig. 21 The $\mathrm{YDH} / \mathrm{SiO}_{2}$ DBR characteristics showing (a) absorbance spectra comparison for $\mathrm{YDH}$ and $\mathrm{HfO}_{2}$ single-layer films in the UV regime, (b) Tauc plot indicates the energy bandgap for $\mathrm{YDH}$ and $\mathrm{HfO}_{2}$ single-layer films, and (c) reflectivity spectrum for 15 periods DBR and the transmission spectrum for a single-layer YDH films with the inset shows the cross-sectional SEM of the DBR structure. Reproduced with permission from Ref. 144.

Despite the advantages of the UV-dielectric DBRs, e.g., extensive material choices, fabrication flexibility, highly reflective, high RI contrast, and the wide stopband, the major drawback is that they are not electrically conductive and the thermal conductivity is mostly low. A complex current injection device scheme, e.g., intracavity ${ }^{132,134}$ or flip-chip, ${ }^{138}$ is required to pump a LEDs with dielectric DBRs electrically. Moreover, the poor thermal conductivity of dielectric DBRs deters the dissipation of heat generated during lasing operation. To overcome limitations, epitaxially grown UV-nitride DBRs have been developed using lattice-mismatched material [(Al)GaN/Al(Ga)N-based, e.g., GaN/AlN, ${ }^{146}$ GaN/AlGaN, ${ }^{147-150}$ AlGaN/AlGaN, ${ }^{151} \mathrm{AlGaN} /$ AlN, ${ }^{152-154}$ and boron-containing nitride, i.e., BAIN/AlN ${ }^{155}$ ], lattice-matched material (AlInN/AlGaN), ${ }^{156-159}$ and nitride-porous (AlGaN/air) material. ${ }^{135}$ The advantage of the UVnitride DBRs is that they can be monolithically grown and doped (either $p$ - or $n$-doped for electrical pumping), and they are furthermore thermally conductive. However, obtaining crack-free DBRs is challenging due to the limitation in crystal growth (substrate compatibility, i.e., latticeand thermal expansion-mismatch, and accumulation of tensile stress) and inadequate RI contrast, i.e., higher periods are required which is challenging for crystal growth in addition to the narrow DBR stopband. It is becoming more critical when the wavelength is shorter (at UV-C), where $\mathrm{AlGaN} / \mathrm{AlGaN}$ DBR needs to be used to avoid absorption issue, but at the same time facing more difficulty in growing high-quality crystal (penalty of higher Al composition) and reduction in RI contrast. Recently, several strain-management techniques (as summarized in Table 7 and shown in Fig. 22) have been demonstrated for growing lattice-mismatched UV-nitride DBRs to suppress the cracks formation and to reduce the mismatch-induced tensile stress. 
Alias et al.: Review of nanophotonics approaches using nanostructures...

Table 7 Strain management techniques demonstrated for lattice-mismatched UV-nitride DBRs.

\begin{tabular}{|c|c|c|c|c|c|c|c|}
\hline Technique & Material system & $\begin{array}{l}\text { Number } \\
\text { of period }\end{array}$ & $\begin{array}{l}\text { Reflectivity } \\
\text { (\%) }\end{array}$ & $\begin{array}{l}\text { Center } \\
\text { wavelength } \\
(\mathrm{nm})\end{array}$ & $\begin{array}{l}\text { Stopband } \\
(\mathrm{nm})\end{array}$ & Remark & Ref. \\
\hline GaN/AIN SLs & $\begin{array}{l}\text { GaN/AIN on GaN } \\
\text { template }\end{array}$ & 20 & 97.0 & 399 (UVA) & 14 & $\begin{array}{l}\text { Mirror } \\
\text { only }\end{array}$ & 146 \\
\hline $\begin{array}{l}\text { AIN/AIGaN double } \\
\text { buffer }\end{array}$ & $\begin{array}{c}\mathrm{GaN} / \mathrm{Al}_{0.2} \mathrm{Ga}_{0.8} \mathrm{~N} \\
\text { on } 6 \mathrm{H}-\mathrm{SiC} \text { substrate }\end{array}$ & 30 & 92.0 & 395 (UVA) & 14 & $\begin{array}{l}\text { Mirror } \\
\text { only }\end{array}$ & 147 \\
\hline $\begin{array}{l}\text { Thin GaN single } \\
\text { buffer }\end{array}$ & $\begin{array}{l}\mathrm{GaN} / \mathrm{Al}_{0.34} \mathrm{Ga}_{0.66} \mathrm{~N} \\
\text { on } \mathrm{GaN} \text { template }\end{array}$ & 35 & 96.0 & 390 (UVA) & 14 & $\begin{array}{l}\text { Mirror } \\
\text { only }\end{array}$ & 148 \\
\hline $\begin{array}{l}\text { AIN/GaN/AIN } \\
\text { multilayer }\end{array}$ & $\begin{array}{l}\mathrm{GaN} \mathrm{Al}_{0.27} \mathrm{Ga}_{0.73} \mathrm{~N} \\
\text { on } \mathrm{GaN} \text { template }\end{array}$ & 20 & 90.0 & 384 (UVA) & 18 & $\begin{array}{l}\text { Mirror } \\
\text { only }\end{array}$ & 149 \\
\hline AIN interlayer & $\begin{array}{l}\mathrm{GaN} / \mathrm{Al}_{0.2} \mathrm{Ga}_{0.8} \mathrm{~N} \\
\text { on } \mathrm{GaN} \text { template }\end{array}$ & 60 & 99.1 & 378 (UVA) & - & $\begin{array}{l}\text { Mirror } \\
\text { only }\end{array}$ & 150 \\
\hline GaN interlayer & $\begin{array}{l}\mathrm{Al}_{0.04} \mathrm{Ga}_{0.96} \mathrm{~N} / \mathrm{Al}_{0.3} \mathrm{Ga}_{0.7} \mathrm{~N} \\
\text { on AIN template }\end{array}$ & 45 & 95.4 & 368 (UVA) & 15 & $\begin{array}{l}\text { Mirror } \\
\text { only }\end{array}$ & 151 \\
\hline $\begin{array}{l}\text { Thick AIN single } \\
\text { buffer }\end{array}$ & $\begin{array}{c}\mathrm{Al}_{0.43} \mathrm{Ga}_{0.57} \mathrm{~N} / \mathrm{AIN} \\
\text { on sapphire substrate }\end{array}$ & 29.5 & 99.0 & 356 (UVA) & 20 & $\begin{array}{l}\text { Mirror } \\
\text { only }\end{array}$ & 152 \\
\hline \multirow[t]{2}{*}{$\begin{array}{l}\text { Wider energy } \\
\text { bandgap }\end{array}$} & BAIN/AIN on AIN template & 18 & 82 & 311 (UVB) & 20 & $\begin{array}{l}\text { Mirror } \\
\text { only }\end{array}$ & 155 \\
\hline & & 24 & 60 & 282 (UVB) & 16 & $\begin{array}{l}\text { Mirror } \\
\text { only }\end{array}$ & 155 \\
\hline Trilayer period & $\begin{array}{c}\mathrm{Al}_{0.5} \mathrm{Ga}_{0.5} \mathrm{~N} / \mathrm{Al}_{0.96} / n_{0.04} \mathrm{~N} / \\
\mathrm{Al}_{x} / \mathrm{n}_{0.02} \mathrm{Ga}_{0.98-x} \mathrm{~N} \\
\text { on AIGaN template }\end{array}$ & 20 & 86 & 274 (UVC) & 16 & $\begin{array}{l}\text { Mirror } \\
\text { only }\end{array}$ & 160 \\
\hline Balanced strains & $\begin{array}{c}\mathrm{Al}_{0.65} \mathrm{Ga}_{0.35} \mathrm{~N} / \mathrm{AIN} \text { on } \mathrm{AIN} \\
\text { template }\end{array}$ & 25.5 & 97.7 & 273 (UVC) & 9 & $\begin{array}{l}\text { Mirror } \\
\text { only }\end{array}$ & 153 \\
\hline $\begin{array}{l}\text { AIN LT } \\
\text { renucleation }\end{array}$ & $\begin{array}{c}\mathrm{Al}_{0.65} \mathrm{Ga}_{0.35} \mathrm{~N} / \mathrm{AIN} \text { on AIN } \\
\text { template }\end{array}$ & 15.5 & 97.7 & 272 (UVC) & 9 & $\begin{array}{l}\text { Mirror } \\
\text { only }\end{array}$ & 154 \\
\hline
\end{tabular}

In Fig. 22(a), a crack-free 20 periods of GaN/AlGaN DBR is realized by inserting three sets of Ga/AlGaN superlattices (SLs) at several positions during the DBR growth. ${ }^{146}$ The reflectivity at $399 \mathrm{~nm}$ wavelength is $\sim 97 \%$. A similar technique was demonstrated by Moudakir et al., ${ }^{149}$ where a multilayer (can be considered as SLs) of AlN/GaN/AIN was inserted at the midpoint of a GaN/AlGaN DBR to suppress the crack generation. For the crack suppression using the buffering technique, Someya and Arakawa ${ }^{148}$ [Fig. 22(b)] found that a highly reflective and crack-free GaN/AlGaN DBR can be grown as long as the thickness of the GaN buffer layer is $<400 \mathrm{~nm}$. Using a thick AlN buffer layer $(\sim 1 \mu \mathrm{m})$, a highly reflective and crack-free DBR based on AlGaN/AlN with 29.5 periods was obtained at 356-nm operating wavelength [see Fig. 22(c)]. ${ }^{152}$ The use of a thick AlN buffer layer keeps the AlGaN layers in compressive stress, whereas the AlN layers were strain-free. A double-layer buffer (using AlN/AlGaN) was also proposed to grow a crack-free and highly reflective GaN/AlGaN DBR on a SiC substrate. ${ }^{147}$

Additionally, an interlayer of GaN or AlN at any point of the UV-nitride DBR structure can be utilized to suppress the crack existence in UV-nitride DBR. Using a thin interlayer, the existence of tensile strain can be compensated. ${ }^{150}$ Figure 22(d) shows that a crack-free AlGaN/AlGaN DBR can be realized after a GaN interlayer is grown after the initial growth of the AlN template on a sapphire substrate. ${ }^{151} \mathrm{~A}$ highly reflective DBR is obtained using 45 periods. The crack suppression is still well managed for a thicker GaN/AlGaN DBR (with 60 periods) using few AlN interlayers at several midpoints during the DBR growth. ${ }^{150}$ In Fig. 22(e), Abid et al. ${ }^{155}$ reported a boron-containing nitride DBR using wider energy bandgap material in the form of BAIN/AIN DBR. This material system has higher RI contrast, which resulted in 

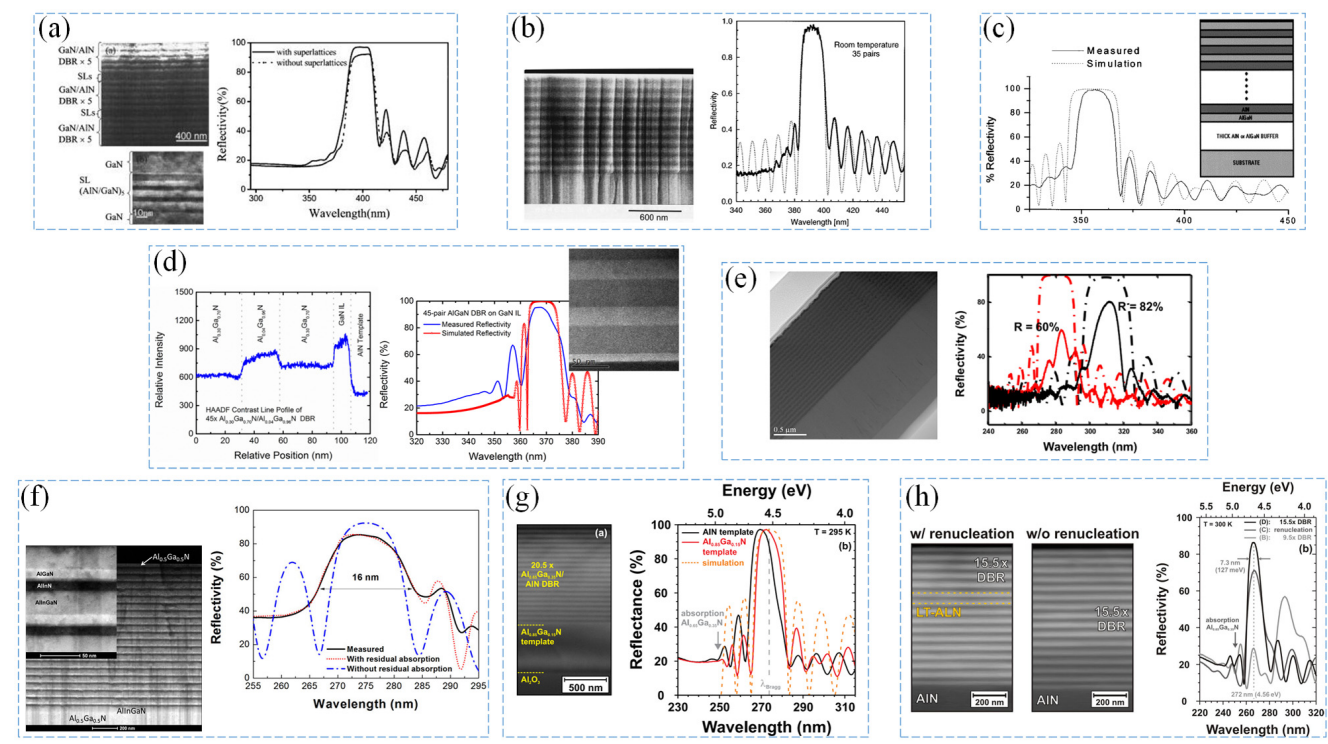

(h)

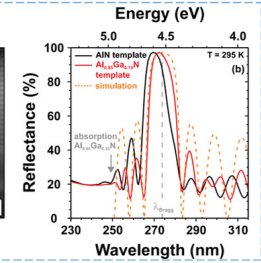

Wavelength $(\mathrm{nm})$

Fig. 22 Various strain-management techniques demonstrated for lattice-mismatched nitrideDBRs resulted in high reflectivity mirror at UV wavelengths and crack-free DBR structure as indicated by the cross-sectional SEM micrographs. (a) GaN/AIN SLs. Reproduced with permission from Ref. 146. (b) Thin GaN single buffer. Reproduced with permission from Ref. 148. (c) AIN single buffer. Reproduced with permission from Ref. 152. (d) GaN interlayer. Reproduced with permission from Ref. 151. (e) Wider energy bandgap. Reproduced with permission from Ref. 155. (f) Trilayer period. Reproduced with permission from Ref. 160. (g) Balanced strains. Reproduced with permission from Ref. 153. (h) AIN-LT nucleation. Reproduced with permission from Ref. 154.

a wider stopband (16 to $20 \mathrm{~nm}$ ) and exhibits less optical absorption than the AlGaN-based DBRs. However, obtaining a high-quality BAlN layer is challenging, which explains the low reflectivity characteristic of the grown DBRs. Recently, several UV-nitride DBRs were demonstrated for UV-C spectral range using advanced techniques, e.g., trilayer period, ${ }^{160}$ balanced strains, ${ }^{153}$ and AlN low-temperature (LT) renucleation. ${ }^{154}$ In the technique of trilayer period [Fig. 22(f)], a period of the DBR consists of three layers, i.e., AlGaN, AlInN, and AlInGaN was used to accommodate the lattice mismatch instead of simply using bilayer, which is typical for a DBR. As for the balanced strains technique [Fig. 22(g)], a crack-free AlGaN/AlN DBR is obtained by counterbalancing the tensile and compressive stress build-up (using a certain $\mathrm{Al}$ composition). As for the AlN low-temperature (LT) renucleation [Fig. 22(h)], an Al layer (grown at LT) was inserted at the midpoint of an AlGaN/AIN DBR structure. All of these advanced UV-nitride DBRs were highly reflective at sub-280-nm wavelength. However, the stopbands were considered narrow ranging from 9 to $16 \mathrm{~nm}$.

To overcome the lattice-mismatch and strain limitations in the UV-nitride DBRs, nearly lattice-matched DBRs using AlInN/AlGaN material system have been demonstrated (summarized in Table 8) as an alternative approach. Figures 23(a) and 23(b) exhibit a UV-A and UV-B latticematched nitride DBRs with a peak reflectivity at 361- and 246-nm wavelength, respectively. A wider DBR stopband (18 to $20 \mathrm{~nm}$ ) was obtained for AlInN/AlGaN DBR compared with GaN/ AlGaN DBR due to higher RI contrast. Although crack-free DBRs can be realized using a lattice-matched material system, the AlInN/AlGaN-based DBRs faced complexity during the growth due to the needs of numerous temperature ramping processes (thus resulting in long growth time) and inhomogeneity of In atoms distribution at such growth temperature. ${ }^{155}$ Furthermore, all of the AlInN/AlGaN-based UV-DBRs can only be grown on AlGaN or AIN templates due to the large built-up strain in the case of GaN templates.

Recently, a technique using nitride-porous, i.e., a combination of epitaxial growth and chemically wet-etching resulting in AlGaN/air, was reported for UV-nitride DBRs. In Fig. 24(a), the cross-sectional SEM image shows the alternating AlGaN epilayer (high RI layer) and porous structure (low RI layer) formed after the etching process. The reflectivity of the AlGaN/porous 
Alias et al.: Review of nanophotonics approaches using nanostructures...

Table 8 Examples of UV-nitride DBRs utilizing lattice-matched material combination.

\begin{tabular}{|c|c|c|c|c|c|c|}
\hline Material system & $\begin{array}{l}\text { Number } \\
\text { of period }\end{array}$ & $\begin{array}{l}\text { Reflectivity } \\
(\%)\end{array}$ & $\begin{array}{c}\text { Center } \\
\text { wavelength } \\
(\mathrm{nm})\end{array}$ & $\begin{array}{l}\text { Stopband } \\
(\mathrm{nm})\end{array}$ & Remark & Ref. \\
\hline $\begin{array}{l}\mathrm{Al}_{0.85} \mathrm{In}_{0.15} \mathrm{~N} / \mathrm{Al}_{0.2} \mathrm{Ga}_{0.8} \mathrm{~N} \\
\text { on AlGaN template }\end{array}$ & 45 & 99.0 & 368 (UVA) & 18 & $\begin{array}{l}\text { Mirror } \\
\text { only }\end{array}$ & 156 \\
\hline $\begin{array}{l}\mathrm{Al}_{0.85} \mathrm{In}_{0.15} \mathrm{~N} / \mathrm{Al}_{0.2} \mathrm{Ga}_{0.8} \mathrm{~N} \\
\text { on AlGaN template }\end{array}$ & 35 & 99.0 & 361 (UVA) & 18 & $\begin{array}{l}\text { Mirror } \\
\text { only }\end{array}$ & 157 \\
\hline $\begin{array}{l}\mathrm{Al}_{0.85} \mathrm{In}_{0.15} \mathrm{~N} / \mathrm{Al}_{0.2} \mathrm{Ga}_{0.8} \mathrm{~N} \\
\text { on AlGaN template }\end{array}$ & 35 & 99.5 & 343 (UVA) & 20 & $\begin{array}{l}\text { Mirror } \\
\text { only }\end{array}$ & 158 \\
\hline $\begin{array}{l}\mathrm{Al}_{0.98} \mathrm{In}_{0.02} \mathrm{~N} / \mathrm{Al}_{0.77} \mathrm{Ga}_{0.23} \mathrm{~N} \\
\text { on AIN template }\end{array}$ & 13.5 & 83.9 & 246 (UVC) & 18 & $\begin{array}{l}\text { Mirror } \\
\text { only }\end{array}$ & 159 \\
\hline
\end{tabular}

(a)

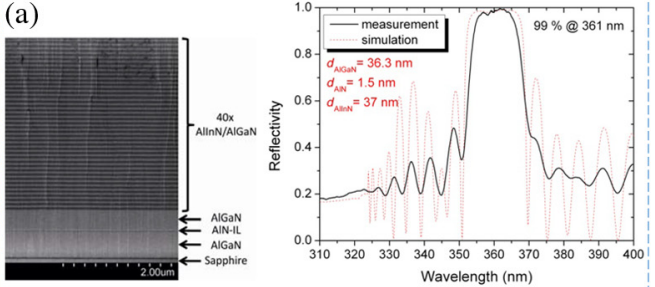

(b)

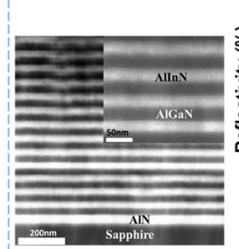

Fig. 23 Examples of lattice-matched UV-nitride DBRs using AllnN/AIGaN material system showing (a) peak reflectivity at 361-nm wavelength, reproduced with permission from Ref. 157, and (b) peak reflectivity at 246-nm wavelength, reproduced with permission from Ref. 159.
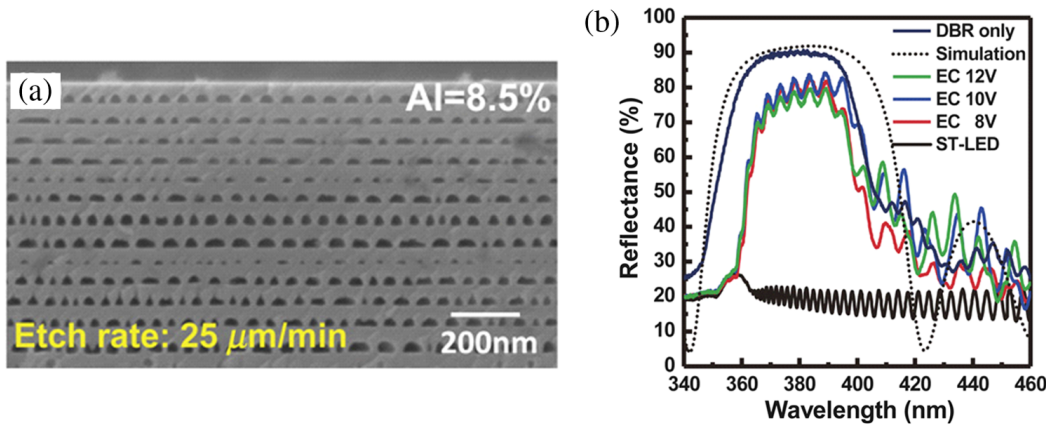

Fig. 24 Demonstration of nitride/porous UV-DBR showing (a) alternating AIGaN epilayer and the porous structure and (b) reflectance spectra of the DBR. Reproduced with permission from Ref. 135.

DBR was measure to be as high as $90 \%$ with a peak wavelength of $379 \mathrm{~nm}$ as shown in Fig. 24(b). A wide DBR stopband of $\sim 37.2 \mathrm{~nm}$ is achieved because of the higher RI contrast of nitride and air. The high RI contrast also resulted in a highly reflective DBR by only using 12 periods of AlGaN/air. Nevertheless, there are still limitations for nitride-porous DBRs, e.g., maintaining mechanical support for the porous layer, process scalability, time-consuming etching, and realizing current injection.

\subsubsection{2-D Photonic crystals: in-plane pattern lattices}

Kashima et al. ${ }^{161}$ reported an AlGaN-based UVC-LED emitting at 283-nm wavelength with an EQE as high as $10 \%$ using PhCs etched into the top p-contact as shown schematically in Fig. 25(a). The cross-sectional STEM for the PhC structure is shown in Fig. 25(b). 
(a)

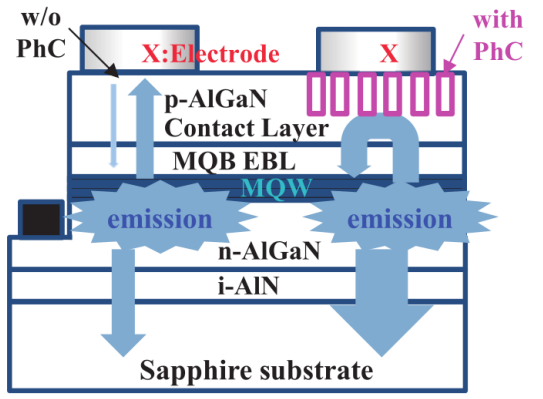

(b)

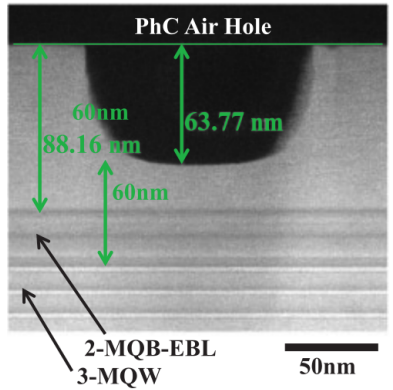

Fig. 25 (a) AIGaN UV-LED structure with and without a PhC. (b) Cross-sectional STEM image of the PhC. Reproduced with permission from Ref. 161.

Furthermore, the advantage of using $\mathrm{PhCs}$ to improve the device performance has been reported for AlInGaN/AlInGaN-based LEDs emitting at $333-{ }^{162}$ and $340-\mathrm{nm}^{163}$ wavelengths. An enhancement in the output power by a factor of 2.5 is found for the LED (emitting at $333 \mathrm{~nm}$ ) with a PhC compared with the control LED without a PhC. For the UV-LED emitting
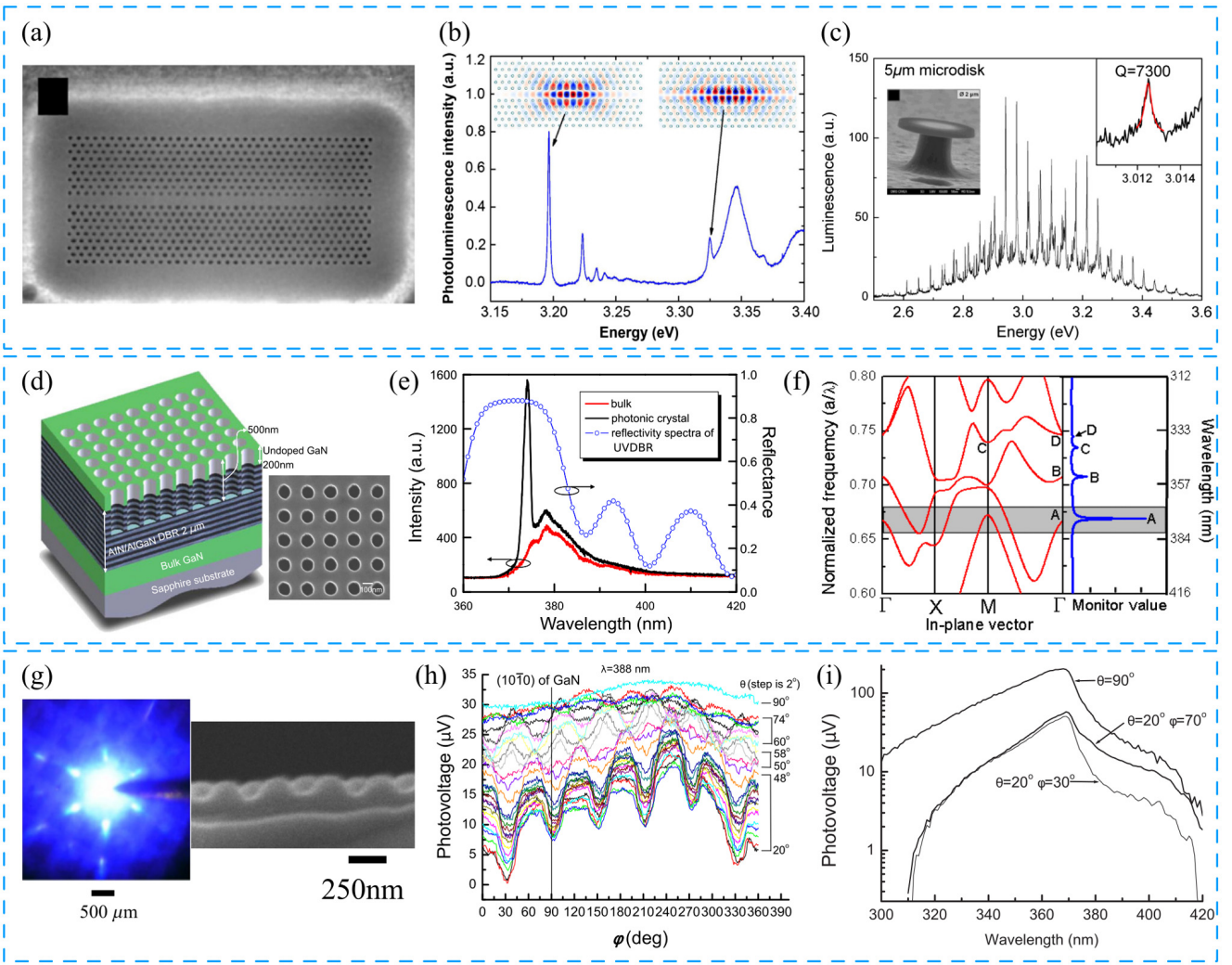

Fig. 26 (a) Top-view SEM image of the AIN PhCs waveguide cavity. (b) PL spectrum of an AIN width modulated waveguide cavity and the magnetic field amplitude profiles of the fundamental even and odd cavity modes. (c) PL of a 5- $\mu \mathrm{m}$-diameter microdisc with AIN QDs. The insets show SEM image of a $2-\mu \mathrm{m}$ microdisc on a Si post; and a zoomed part of PL at one resonance. Reproduced with permission from Ref. 165. (d) Structure of the square PhCs lattice etched into DBR (left) and the top view SEM image (right). (e) Measured reflectivity spectrum of the AIN/AIGaN DBR structure and PL spectra of bulk GaN and patterned UV-DBR structure. Reproduced with permission from Ref. 166. (f) The corresponding band diagram and luminescence spectra (simulation). Reproduced with permission from Ref. 167. (g) Photo of LED with GaN nanostructure pattern emission (left) and SEM cross section (right). (h) Photovoltage scan at the wavelength of 388-nm versus the incidence angle $\theta$ and azimuth angle $\varphi$ values. (i) Photovoltage versus wavelength at different $(\theta, \varphi)$ values. Reproduced with permission from Ref. 168. 
at $340 \mathrm{~nm}$, by integrating the PhCs into the device structure, the forward voltage decreased by $\sim 1.6 \mathrm{~V}$ and the power enhanced by $95 \%$ at $20 \mathrm{~mA} .{ }^{163}$

Other $\mathrm{PhC}$ works base their research on improving the $\mathrm{PhC}$ optical properties, e.g., in the case of exploring the effect of PhCs on time-resolved $333 \mathrm{~nm}$ UV-LED EL spectra ${ }^{164}$ and microresonators. ${ }^{165}$ Specifically, an in-plane $\mathrm{PhC}$ waveguide with a line defect is made [Fig. 26(a)]. Additionally, central holes are displaced by a maximum of $12 \mathrm{~nm}$ laterally, and the lattice periodicity is $170 \mathrm{~nm}$. The experimental PL data are shown in Fig. 26(b) together with the mode profiles obtained by 3D-FDTD simulation, manifesting one sharp high fundamental mode resonance in near UV and lower one in UV-A regime. Such studies and the PhCs concept can be successfully applied, for example, in AlN microdisks with embedded QDs exhibiting a tunable resonance spectrum consisting of numerous modes, highlighting the high-quality $Q$ factors [Fig. 26(c)]. PhCs also have been successfully implemented in devices for the use as PDs. ${ }^{168}$ Figure 26(g) shows a PD/LED emission with hexagonal beam pattern due to the hexagonal lattice pattern $\mathrm{PhC}$ on top of the device. It is capable of sensing the absolute photovoltage $(\mathrm{PV})$, and it is revealed that PV strongly depends on the device orientation with respect to the detected beam's direction, i.e., on the incidence angle $\theta$ and azimuth angle $\varphi$, as seen in Fig. 26(h). There we can see the sixfold periodic pattern as $\varphi$ rotates a full circle, as is suspected due to the hexagonal type symmetry of the PhC that couples the light into PD layers. Last, the responsivity curves versus wavelength at different angles are shown in Fig. 26(i), showcasing the capability of PV difference sense. Thus, one of the possible applications of this PD includes orientation/direction-aware and localization (as in positioning) solutions.

\subsubsection{3-D Photonic crystals: hybrid structures}

Figure 26(d) shows an example of 3-D PhC on the GaN-based sample for UV regime. ${ }^{166,167}$ Although this may not be considered a true general 3-D case, it is a hybrid structure consisting of a PhC partially etched through an AlN/AlGaN DBR. It establishes complex light-structure interaction that depends at a minimum on three orthogonal spatial degrees of freedom, thus 3-D. As can be seen from the results [Fig. 26(e)], the PL signal is greatly enhanced. The PL enhancement is 5 times compared with DBR without the PhC. The peak wavelength of this structure is $374 \mathrm{~nm}$-inside the stopband of DBR. The UV-DBR helps to eliminate resonant modes outside the DBR high reflection region, which in turn decreases energy waste. The simulated luminescence spectrum [Fig. 26(f)] confirms the experimental resonant peak with high accuracy. Table 9 summarizes the reported UV devices utilizing 2-D or 3-D PhCs. The table compares typical device structure, working wavelength, and a general enhancement factor.

\subsection{Nanogratings}

Nanogratings (NGs), when properly implemented on a surface of a light-emitting device, is a way to improve the LEE. Alias et al. ${ }^{172}$ demonstrated NGs for UV-LED as NWs gratings around the device lateral configuration as shown in Fig. 27(a). The NWs gratings were patterned using gas-assisted FIB (GAFIB) etching with $I_{2}$ halide gaseous precursor. The GAFIB etching can enhance etching, reduce surface damage, and decrease the redeposition effect. ${ }^{173,174}$ Figure 27(b) shows a substantial increase in the LOP detected from the top. Figure 27(c) shows the calculated intensity pattern of the devices with and without DBR NGs.

\subsubsection{Subwavelength gratings}

Subwavelength gratings (SWGs) are not only applied to enhance the light output, but also to control the polarizing properties. Although SWGs support only zeroth-order diffraction, they still exhibit polarization dependence. When an SWG is designed as a polarizer, one often targets to achieve high polarization selectivity: ideally, one polarization component is completely cutoff, and the other one is diffracted with $100 \%$ efficiency. The Ge SWG polarizer that is shown in Fig. 28(a) is fabricated with extinction ratio (ER) of 15 to $17.4 \mathrm{~dB}$ in UV-A range with a low aspect ratio (AR). ${ }^{175}$ 
Alias et al.: Review of nanophotonics approaches using nanostructures...

Table 9 Summary of UV-photonic devices with 2-D/3-D PhCs approaches.

\begin{tabular}{|c|c|c|c|c|c|}
\hline Technique & Device & $\begin{array}{l}\text { Device structure } \\
\text { (top-down) }\end{array}$ & Device characteristic & Enhancement & Ref. \\
\hline \multirow[t]{7}{*}{ 2-D PhCs } & $\begin{array}{l}\text { UV-C LED } \\
\text { (MOVPE) }\end{array}$ & $\begin{array}{c}\text { PhCs p-AIGaN } 90 \mathrm{~nm} \\
(a \sim 250 \mathrm{~nm} r / a=0.2 \\
h=64 \mathrm{~nm}) \\
\text { MQW } 3 \times \text { AlGaN: } \\
1.5 / 7 \mathrm{~nm}\end{array}$ & $\begin{array}{l}\text { Emitting at } 283 \mathrm{~nm} \\
\text { (electrical pumping) } \\
\text { IQE } \sim 50 \% \text { to } 60 \%\end{array}$ & $\begin{array}{l}\sim 23 \% \text { to } \\
25 \% \text { (EQE) }\end{array}$ & 161 \\
\hline & $\begin{array}{l}\text { NUV-LED } \\
\text { (MOCVD) }\end{array}$ & $\begin{array}{c}\mathrm{SiO}_{2} \mathrm{PhCs} \sim 900 \mathrm{~nm} \\
(d=600 \mathrm{~nm} r / a=0.35 \\
h=100 \mathrm{~nm}) \\
\mathrm{MQW} 10 \times \mathrm{InGaN} / \mathrm{GaN}\end{array}$ & $\begin{array}{l}\text { Emitting at } \sim 398 \mathrm{~nm} \\
\text { (electrical pumping) }\end{array}$ & $\begin{array}{l}27.7 \%(E L) \\
\text { at } 20 \mathrm{~mA}\end{array}$ & 169 \\
\hline & $\begin{array}{l}\text { UV-A LED } \\
\text { (MOCVD) }\end{array}$ & $\begin{array}{c}\text { PhCs p-GaN } 150 \mathrm{~nm} \\
(a=600 \mathrm{~nm} r / a \approx 0.17 \\
h \sim 185 \mathrm{~nm}) \\
\text { MQW } 2 \times \text { AllnGaN: } \\
2.5 / 2.5 \mathrm{~nm}\end{array}$ & $\begin{array}{l}\text { Emitting at } 333 \mathrm{~nm} \\
\text { (electrical pumping) }\end{array}$ & $\begin{array}{l}2.5 \times(E L) \\
\text { at } 20 \mathrm{~mA}\end{array}$ & 162,170 \\
\hline & $\begin{array}{l}\text { UV-A LED } \\
\text { (MOCVD) }\end{array}$ & $\begin{array}{c}\text { PhCs p-GaN } 200 \mathrm{~nm} \\
(a=700 \mathrm{~nm} r / a \approx 0.21 \\
h \sim 200 \mathrm{~nm}) \\
\text { QW AllnGaN: } 2 / 3 \mathrm{~nm}\end{array}$ & $\begin{array}{l}\text { Emitting at } 340 \mathrm{~nm} \\
\text { (electrical pumping) }\end{array}$ & $\begin{array}{l}95 \%(E L) \\
\text { at } 20 \mathrm{~mA}\end{array}$ & 162,163 \\
\hline & $\begin{array}{l}\text { UV-B LED } \\
\text { (MOCVD) }\end{array}$ & $\begin{array}{c}\text { PhCs p-GaN } \\
(a=500 \mathrm{~nm} r / a=0.3) \\
\text { [see the above } \\
\text { two structures] }\end{array}$ & $\begin{array}{l}\text { Emitting at } 299 \mathrm{~nm} \\
\text { (electrical pumping) }\end{array}$ & $2.9 \times(E L)$ & 162,171 \\
\hline & $\begin{array}{l}\text { UV-A p-n PD/LED } \\
\text { (MOCVD) }\end{array}$ & $\begin{array}{c}\text { PhCs p-GaN } 110 \mathrm{~nm} \\
(a=300 \mathrm{~nm} r / a=0.25 \\
h \sim 100 \mathrm{~nm}) \\
\text { QW InGaN } 2 \mathrm{~nm}\end{array}$ & LED emission at $433 \mathrm{~nm}$ & $\begin{array}{l}22 \times \text { at } 388 \mathrm{~nm} \\
\text { for } 20 \mathrm{deg} \\
\text { incidence } \\
\text { and } 255 \text { deg in- } \\
\text { plane angle }\end{array}$ & 168 \\
\hline & - & $\begin{array}{c}\text { AIN modified } \\
\text { waveguide } 35 \mathrm{~nm} \\
(a=170 \mathrm{~nm} r / a=0.27 \\
h=75 \mathrm{~nm}) \\
\text { GaN QDs }\end{array}$ & $\begin{array}{c}\text { Resonance tunable } \\
\text { by } a \text { and } r / a \text { down } \\
\text { to } 290 \mathrm{~nm}\end{array}$ & $\begin{array}{c}\text { Fundamental } \\
\text { mode } \\
\text { PL resonance at } \\
388 \text { and } 373 \mathrm{~nm}\end{array}$ & 165 \\
\hline 3-D PhCs & $(\overline{M O C V D})$ & $\begin{array}{c}\text { PhCs u-GaN } 200 \mathrm{~nm} \\
(a=250 \mathrm{~nm} r / a=0.28 \\
h \sim 500 \mathrm{~nm}) \\
\text { DBR } 25 \text { pairs AlGaN: } \\
45 / 42 \mathrm{~nm}\end{array}$ & $\begin{array}{l}\text { DBR reflectivity } 85 \% \\
\text { stop-band width } \\
15 \mathrm{~nm} \text { at } 375 \mathrm{~nm}\end{array}$ & $\begin{array}{c}5 \times(\mathrm{PL}) \text { compared } \\
\text { to DBR without } \\
\text { PhCs }\end{array}$ & 166,167 \\
\hline
\end{tabular}

Works by Weber et al. show feasibility of efficient wire grid polarizers (WGPs) in UV-B and UV-C regimes. ${ }^{176,177,179,180}$ They have reported different material grating structures, e.g., Ir, ${ }^{177}$ $\mathrm{Si}^{180}$ and $\mathrm{Al}^{176,179,180}$ gratings down to $250 \mathrm{~nm}$ and $\mathrm{W}^{179}$ grating down to 193 -nm wavelength, as seen in Fig. 28(b) with given examples in SEM images of Ir structures on polymer gratings [Fig. 28(c)]. Reference 178 shows insights into WGP design using material complex RI analysis and optical simulations presents $\mathrm{TiO}_{2}$ as a better alternative [Fig. 28(d)] to $\mathrm{W}$ and Ir polarizers for UV-C range. The $\mathrm{TiO}_{2}$-based WGP showed a superior ER of $384(25.8 \mathrm{~dB})$ and transmittance of $10 \%$ at $193 \mathrm{~nm}$ as shown in Fig. 28(e). Another work investigates $\mathrm{Cr}_{2} \mathrm{O}_{3}$ SWGs as WGPs further in depth for UV-C with a grating period equal to $\sim 90 \mathrm{~nm}$ and measured ER of $138(21.4 \mathrm{~dB}){ }^{181}$ The performance was compared with $\mathrm{Al}_{2} \mathrm{O}_{3}, \mathrm{Ta}_{2} \mathrm{O}_{5}, \mathrm{TiO}_{2}$, and $\mathrm{Al}$ gratings at $193 \mathrm{~nm}$. The $\mathrm{Cr}_{2} \mathrm{O}_{3}$ grating showed the highest absorption thus suppressing the TE mode the most while having practically the same transmission as the other materials. Reference 182 by numerical simulation considered AlGaN UV-A LED enhancement and fabricated a structure with a simple Si SWG on top of the LED that resulted in ER of $\sim 16$ and increase in TM polarized EL intensity by $\sim 20 \%$. The same author also reported in Ref. 183 a reverse ER, i.e., s/p ratio of $\sim 4$ at 360-nm wavelength, but by etching the top contact to form a grating structure. 

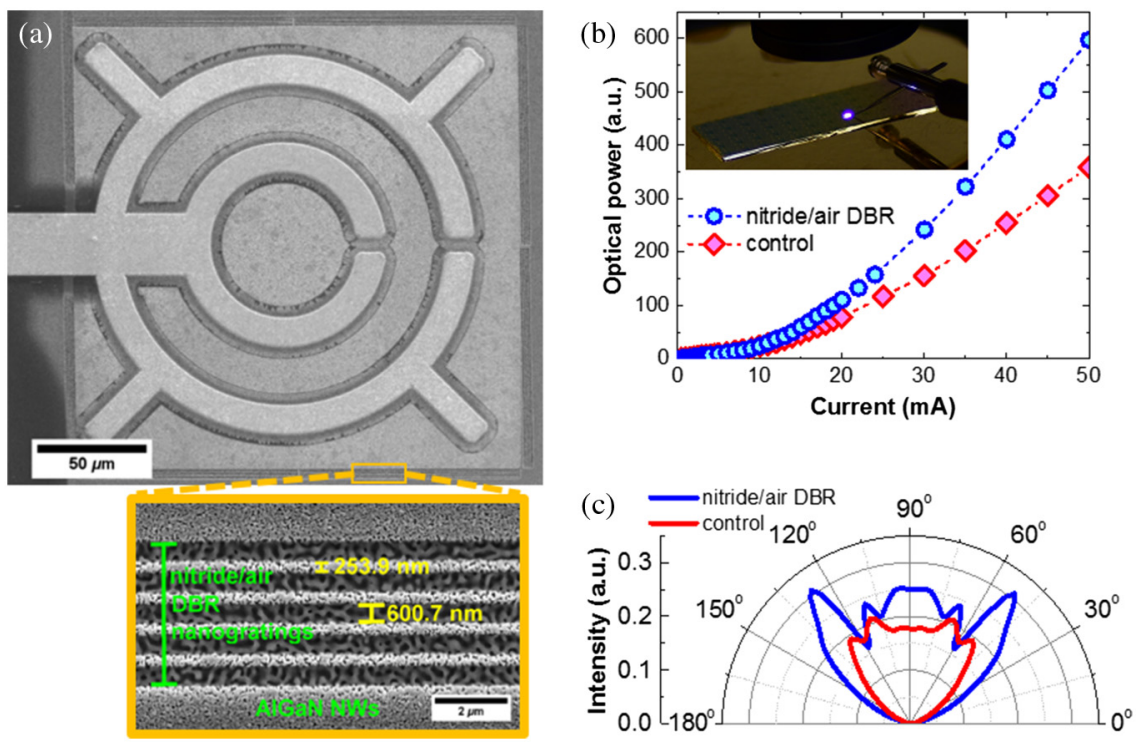

Fig. 27 (a) The top view SEM of the AIGaN NWs LED; the outset: the DBR NGs patterned at all edges of the device showing the dimension measurements of the NGs. (b) The device L-I curve characteristics comparison between the LED with and without DBR NGs (the inset: device with NGs under electrical current injection). (c) Comparison of the calculated polar pattern for both devices. Reproduced with permission from Ref. 172.

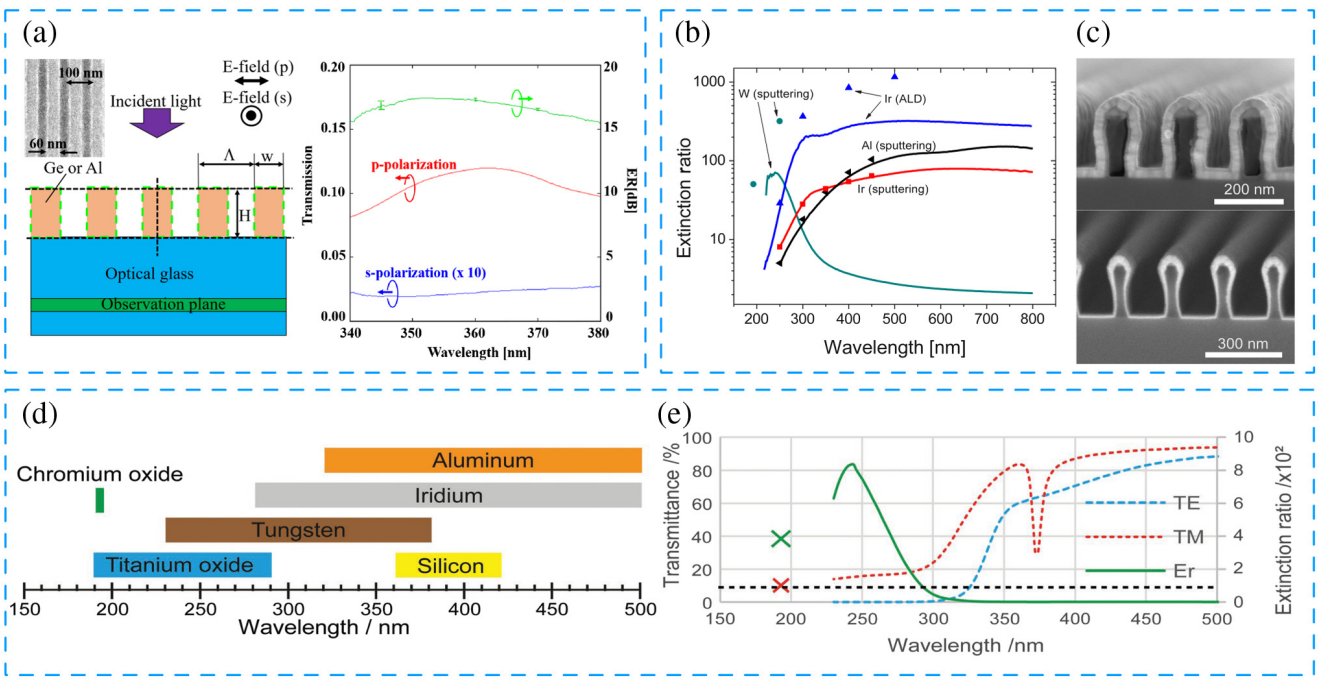

Fig. 28 (a) Schematic of the SWG with top-view SEM image of the fabricated Ge-SWG and the measured transmission and ER of the Ge-SWG. Reproduced with permission from Ref. 175. (b) Measured values of the ER of WGPs. Reproduced with permission from Ref. 176. (c) SEM images of the fabrication process for the WGPs with the overcoated polymer gratings by ALD (top) and by sputter deposition (bottom). Reproduced with permission from Ref. 177. (d) Comparison of application wavelength ranges of several WGPs from various metals and oxides. (e) Measured transmittances and ER of the fabricated titanium dioxide WGP. Reproduced with permission from Ref. 178.

\subsubsection{Other devices and applications}

Recent work demonstrates homogenous NG fabrication by exciting SP polaritons (SPPs) with 266-nm UV fs pulses thus allowing a formation of gratings with a wavelength-dependent period smaller than half of the excitation wavelength, showcasing another possibility of fabricating 


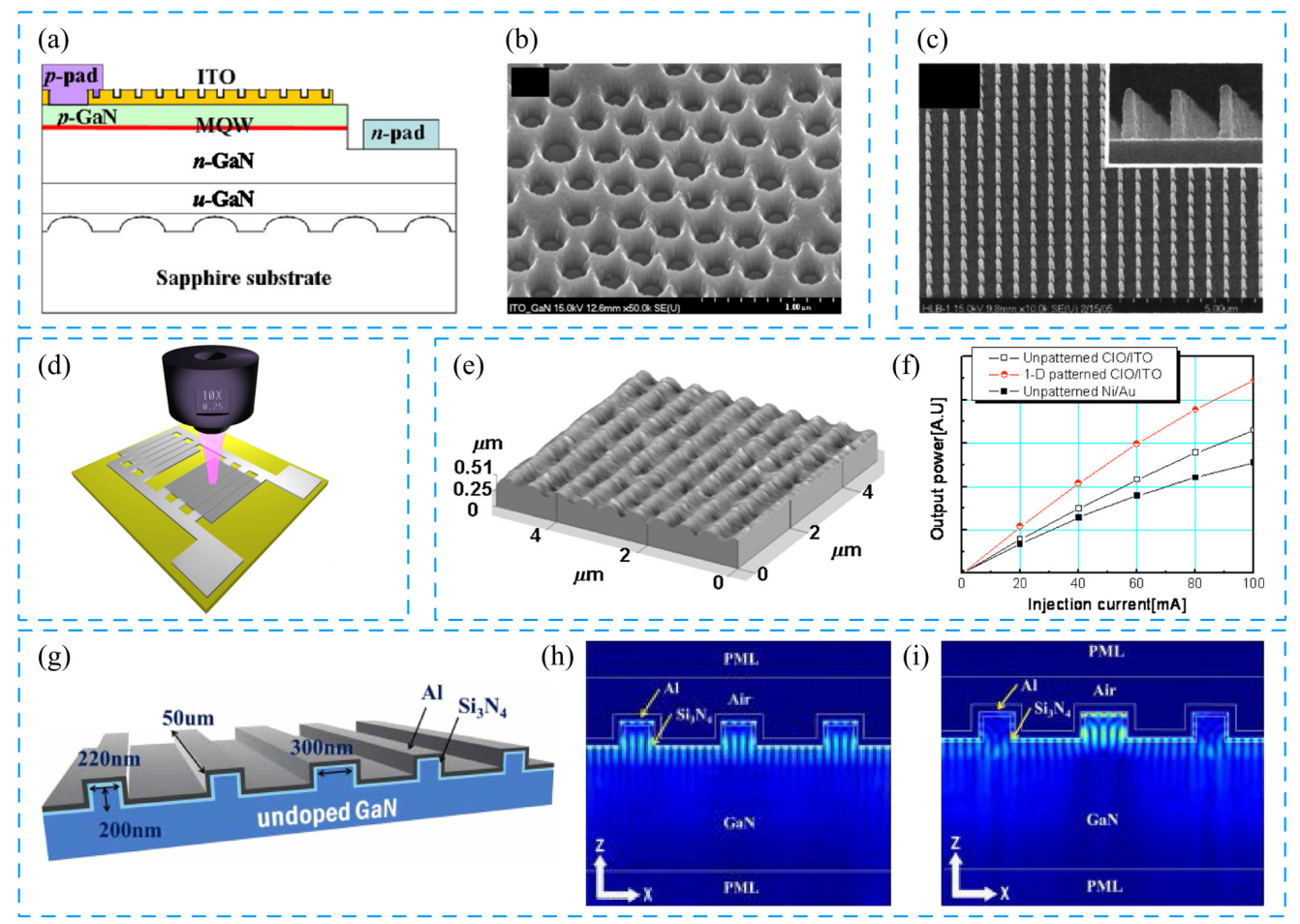

Fig. 29 (a) Schematic of an LED structure with a patterned ITO contact layer. (b) 45-deg tilt SEM image of the hole patterned p-GaN layer. Reproduced with permission from Ref. 186. (c) SEM image of 2-D patterned photoresist, the inset shows enlarged cross-section image. Reproduced with permission from Ref. 187. (d) Drawing of nanostructured MSM PD. Reproduced with permission from Ref. 188. (e) AFM image of 1-D patterned ITO. (f) The L-I characteristics of LEDs fabricated with unpatterned CIO/ITO, 1-D patterned CIO/ITO, and conventional $\mathrm{Ni} / \mathrm{Au}$ contacts. Reproduced with permission from Ref. 189. (g) Schematic of the metal-coated GaN grating structure with defect cavity. (h-i) Simulated electric-field intensity in the metal-coated GaN grating (h) without and (i) with the defect cavity. Reproduced with permission from Ref. 185.

SWGs. ${ }^{184}$ This team managed to achieve gratings with around 50 - to 60 -nm period lengths. Another work ${ }^{185}$ uses SPPs phenomenon to reduce PL threshold by $75 \%$ for lasing in GaN by introducing a defect as a cavity in patterned GaN NG structure with a coated $50-\mathrm{nm} \mathrm{Al}$ metal layer [Figs. 29(g)-29(i)]. Although a simpler approach to improving LEE of UVLEDs is contact patterning in a regular structure like a grating demonstrated in Refs. 189 and 190 [Figs. 29(e)-29(f)]. Other grating patterns, e.g., hexagonal hole pattern and square pattern gratings, were used by Kim et al. ${ }^{186}$ and Leem et al., ${ }^{187}$ respectively, as seen in Figs. 29(a)-29(c).

Large-area soft UV-curing NIL fabrication of NRs for PL enhancement up to 2.5 times was demonstrated in Ref. 191 that can benefit optoelectronic applications. ${ }^{192}$ Yet other unmentioned devices are UV PDs as demonstrated in Ref. 188 [Fig. 29(d)], where an Al metal grating with a slit width of $100 \mathrm{~nm}$ was employed to increase the photocurrent by 8 times at 322-nm illumination and at the same time achieving faster response and decay time of the PD.

The nanograting structures in this section are summarized in Table 10. The table compares typical device structure, working wavelength, and a general enhancement factor.

\subsection{Microdisks and Nanopillars}

Another approach that has been suggested for the development of III-nitride-based UV emitting devices is the use of microdisks and nanopillars. Similar to the previously described NGs and $\mathrm{PhCs}$, microdisks and nanopillars provide a resonant cavity for the light to be confined and amplified. Moreover, both the microdisks and nanopillars act as a micro- and nanoscale 
Alias et al.: Review of nanophotonics approaches using nanostructures...

Table 10 Summary of III-nitrides UV-photonic devices with NGs approaches.

\begin{tabular}{|c|c|c|c|c|c|}
\hline Technique & Device & $\begin{array}{l}\text { Device structure } \\
\text { (top-down) }\end{array}$ & Device characteristic & Enhancement & Ref. \\
\hline \multirow[t]{2}{*}{$\begin{array}{l}\text { Lateral } \\
\text { DBR }\end{array}$} & $\begin{array}{l}\text { UV-A NW } \\
\text { LED (MBE) }\end{array}$ & $\begin{array}{l}\text { Etched DBR } \\
\text { around LED }\end{array}$ & $\begin{array}{l}\text { Emitting at } \sim 343 \mathrm{~nm} \\
\text { (electrical pumping) }\end{array}$ & $\begin{array}{l}\sim 59 \% \text { to } 69 \% \\
\text { (EQE) }\end{array}$ & 172 \\
\hline & & $\begin{array}{c}(d \approx 850 h \sim 380 \\
w \sim 250) \mathrm{nm} \\
\mathrm{MQW} \text { AlGaN: } \\
1 / 3.5 \mathrm{~nm}\end{array}$ & & $\begin{array}{c}\text { FWHM } \\
\sim 15 \rightarrow 12 \mathrm{~nm}\end{array}$ & \\
\hline \multirow[t]{8}{*}{$\begin{array}{l}\text { SWG } \\
\text { polarizer }\end{array}$} & $\begin{array}{l}\text { UV-A LED } \\
\text { (MOCVD) }\end{array}$ & $\begin{array}{c}\text { Si SWG } \\
(d=205 h=90 \\
w=100) \mathrm{nm} \\
\text { QW AIGaN/GaN }\end{array}$ & $\begin{array}{l}\text { Emitting at } 370 \mathrm{~nm} \\
\text { (electrical pumping) } \\
\quad \mathrm{AR}=0.9\end{array}$ & $\begin{array}{l}\sim 20 \%(\mathrm{TM} \text { EL) } \\
\mathrm{ER} \sim 12 \mathrm{~dB}(16 \times) \\
\text { at } 370 \mathrm{~nm}\end{array}$ & 182 \\
\hline & $\begin{array}{l}\text { UV-A LED } \\
\text { (MOCVD) }\end{array}$ & $\begin{array}{l}\text { Patterned p-GaN } 100 \mathrm{~nm} \\
(d=200 h=150 \\
w=140) \mathrm{nm} \\
\text { MQW AllnGaN/AIGaN }\end{array}$ & $\begin{array}{l}\text { Emitting at } 365 \mathrm{~nm} \\
\text { (electrical pumping) } \\
\qquad \mathrm{AR}=1.07\end{array}$ & $\begin{array}{l}\mathrm{ER}^{-1}(\mathrm{~s} / \mathrm{p}) \\
\sim 6 \mathrm{~dB}(4 \times) \\
\text { at } 360 \mathrm{~nm}\end{array}$ & 183 \\
\hline & - & $\begin{array}{c}\text { a-Si grating } \\
(d=140 h=80 w=42) \\
\mathrm{nm} \\
\text { Fused silica substrate }\end{array}$ & $\mathrm{AR}=1.90$ & $\begin{array}{c}\mathrm{ER}=19.5 \mathrm{~dB}(90 \times) \\
w / T=42 \% \text { at } \\
365 \mathrm{~nm}\end{array}$ & 180 \\
\hline & - & $\begin{array}{c}\text { Ge grating } \\
(d=100 h=100 \\
w=60) \mathrm{nm} \\
\text { Optical glass }\end{array}$ & $\mathrm{AR}=1.67$ & $\begin{array}{c}\mathrm{ER}=17.4 \mathrm{~dB}(55 \times) \\
w / T=12 \% \text { at } \\
360 \mathrm{~nm}\end{array}$ & 175 \\
\hline & - & $\begin{array}{c}\text { Ir grating } \\
\qquad(d=100 h=150 \\
w=30) \mathrm{nm} \\
\text { Fused silica substrate }\end{array}$ & $A R=5$ & $\begin{array}{c}\mathrm{ER}=19.4 \mathrm{~dB}(87 \times) \\
w / T=45 \% \text { at } \\
250 \mathrm{~nm}\end{array}$ & 177,176 \\
\hline & - & $\begin{array}{c}\text { W grating } \\
\qquad(d=100 h=150 \\
w=30) \mathrm{nm} \\
\text { Fused silica substrate }\end{array}$ & $\mathrm{AR}=5$ & $\begin{array}{c}\mathrm{ER}=22 \mathrm{~dB}(158 \times) \\
w / T=40 \% \text { at } \\
193 \mathrm{~nm}\end{array}$ & 179,176 \\
\hline & - & $\begin{array}{c}\text { Two } \mathrm{Cr}_{2} \mathrm{O}_{3} \text { gratings } \\
(d \sim 90 h \sim 120 w \sim 36) \\
\text { nm } \\
\text { Quartz substrate }\end{array}$ & $\mathrm{AR} \sim 3.3$ & $\begin{array}{c}\mathrm{ER}=21.4 \mathrm{~dB} \\
(138 \times) \\
w / T=18.6 \% \text { at } \\
193 \mathrm{~nm}\end{array}$ & 181 \\
\hline & - & $\begin{array}{c}\mathrm{TiO}_{2} \text { grating } \\
(d=104.5 h=150 \\
w=26) \mathrm{nm} \\
\text { Fused silica substrate }\end{array}$ & $\mathrm{AR}=5.77$ & $\begin{array}{c}\mathrm{ER}=25.8 \mathrm{~dB} \\
(384 \times) w / T=10 \% \\
\text { at } 193 \mathrm{~nm}\end{array}$ & 178 \\
\hline SW feature & $\begin{array}{l}\text { UV-B MSM } \\
\text { PD } \\
\text { (MOCVD) }\end{array}$ & $\begin{array}{l}\text { Al metal grating }(d=314 \\
h=35 w \approx 105) \mathrm{nm} \text { with } \\
100 \mathrm{~nm} \text { slit; u-GaN } 1 \mu \mathrm{m}\end{array}$ & $\begin{array}{l}\text { Dark current }<1 \text { pA up } \\
\text { to } 7 \mathrm{~V} \text { bias } \\
\mathrm{V} \text { (breakdown) } \sim 15 \mathrm{~V}\end{array}$ & $\begin{array}{l}8 \times \text { (photocurrent) } \\
\text { at } 322 \mathrm{~nm}\end{array}$ & 188 \\
\hline \multirow[t]{2}{*}{$\begin{array}{l}\text { Patterned } \\
\text { top contact }\end{array}$} & $\begin{array}{l}\text { NUV-LED } \\
\text { (MOCVD) }\end{array}$ & $\begin{array}{c}\text { CIO/ITO grating }(d \sim 500 \\
h=100) \mathrm{nm} \mathrm{MQW} \\
\text { InGaN/GaN }\end{array}$ & $\begin{array}{l}\text { Emitting at } 398 \mathrm{~nm} \\
\text { (electrical pumping) }\end{array}$ & $\begin{array}{c}33 \%(E L) w / 1-D \\
\text { and } 48 \% w / 2-D \\
\text { nanopattern }\end{array}$ & 190 \\
\hline & $\begin{array}{l}\text { UV-A LED } \\
\text { (MOCVD) }\end{array}$ & $\begin{array}{c}\text { CIO/ITO grating }(d \sim 500 \\
h=100 w \sim 250) \mathrm{nm} \\
\mathrm{MQW} \operatorname{lnGaN} / \mathrm{GaN}\end{array}$ & $\begin{array}{l}\text { Emitting at } 385 \mathrm{~nm} \\
\text { (electrical pumping) }\end{array}$ & $\begin{array}{l}40 \% \text { (EL) with 1-D } \\
\text { nanopattern }\end{array}$ & 189 \\
\hline $\begin{array}{l}\text { GaN } \\
\text { nanorods }\end{array}$ & - & GaN Sapphire & $\mathrm{PL}$ at $\sim 450 \mathrm{~nm}$ & $\begin{array}{l}\sim 2.5 \times(\mathrm{PL}) \\
\text { compared to } \mathrm{GaN} \\
\text { films structure }\end{array}$ & 191 \\
\hline $\begin{array}{l}\text { Metal- } \\
\text { coated GaN } \\
\text { defect } \\
\text { grating }\end{array}$ & $(\mathrm{MOCVD})$ & $\begin{array}{c}50 \mathrm{~nm} \text { Al metal layer, } \\
\text { grating }(d=840 \\
w=220 h=200) \mathrm{nm}, \mathrm{u}- \\
\text { GaN } 2 \mu \mathrm{m}\end{array}$ & $\begin{array}{l}\text { I(cavity) }=300 \mathrm{~nm} \\
\text { RT PL lasing at } \\
365 \mathrm{~nm}\end{array}$ & $\begin{array}{l}Q=480 \text { and } \\
\text { reduced threshold } \\
\text { power density by } \\
75 \%\end{array}$ & 185 \\
\hline
\end{tabular}


light source, thus becoming a promising approach for applications in UV micro- and nanophotonics. In 2003, Jiang and $\operatorname{Lin}^{193}$ reviewed III-nitride microemitters, then in 2014, Néel et al. ${ }^{165}$ briefly reviewed AlN microdisks, and in 2015, Zhang et al. ${ }^{194}$ reviewed III-nitride microdisk lasers over the UV and visible spectrum. Here, we provide a historical context of a broad set of microdisks and nanopillars structures focusing exclusively on III-nitride UV emitters fabricated by a top-down approach.

\subsubsection{Microdisks}

A semiconductor microdisk laser is a circular resonator cavity formed by top-down fabrication of an active material, i.e., semiconductor material or structure, where both physical and chemical etching are used to pattern the structures and release them into a mushroom-like configuration. The microdisk made from the active material is sustained on a pillar made from the substrate
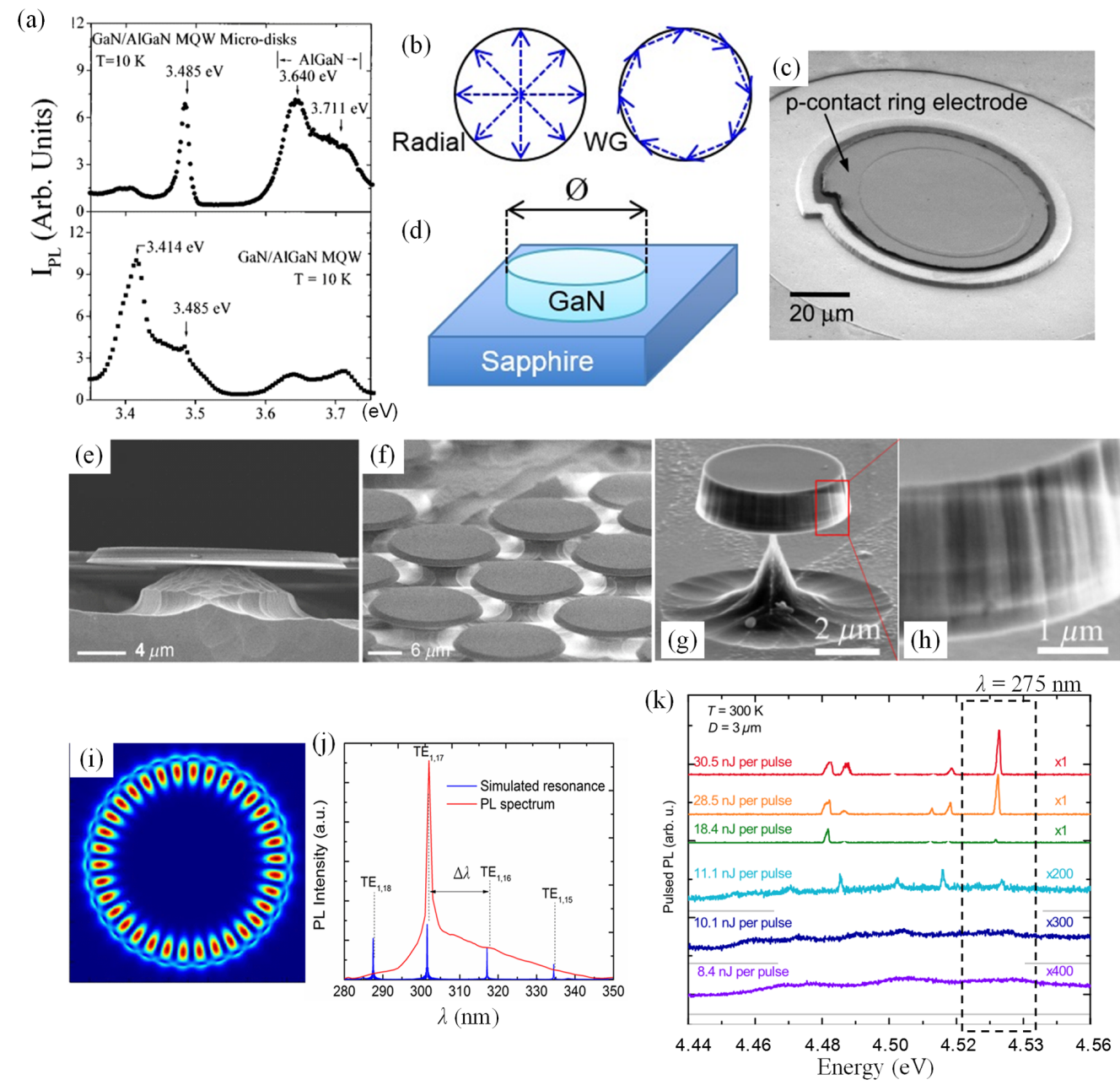

Fig. 30 The development of UV-emitting microdisks from 1997 to 2018. (a) PL spectra of GaN/ AlGaN material before (bottom) and after (top) microdisk fabrication. Reproduced with permission from Ref. 195. (b) Schematic of radial and WG resonant modes in a microdisk. (c) SEM image of the electrically driven directional UV-A microdisk laser. Reproduced with permission from Ref. 196. (d) Schematic of a GaN microdisk in full contact with a sapphire substrate. (e) Lateral and (f) perspective view of freestanding In GaN/GaN-based microdisks on Si substrate. Reproduced with permission from Ref. 197. (g) GaN microdisk on Si post with detail (h) of edge NGs. Reproduced with permission from Ref. 198. (i) FDTD-simulated electric field intensity of a $\lambda=301 \mathrm{~nm}$ WG mode as well as the mode matching with the corresponding PL emission (j). Reproduced with permission from Ref. 199. (k) PL emission of a 3- $\mu \mathrm{m}$ microdisk at different pulse-injection intensities showing lasing at $\lambda \sim 275 \mathrm{~nm}$. Reproduced with permission from Ref. 200. 
material [Figs. 30(e)-30(g)]. Microdisks can also be realized in full contact with the substrate [Figs. 30(c) and 30(d)]. Here, we present and discuss the experimental demonstrations and historical progress toward UV microlasers based on microdisk structures.

The first top-down attempt to realize microdisks of GaN/AlGaN MQW was the work of Mair et al. ${ }^{195}$ Employing pulsed optical pumping under LT condition $(10 \mathrm{~K})$, they were able to compare the PL (continuous and time-dependent) of the GaN/AlGaN material before and after microdisk fabrication [Fig. 30(a)]. This study showed that the recombination lifetime and the quantum efficiency (QE) of the GaN/AlGaN intrinsic transitions, were improved by a factor of 10 after the formation of the microdisks. Additionally, the contribution of the impurity transition of misfit dislocations was drastically reduced. These findings confirmed the influence of microdisks structure on the light emitting properties of GaN-based materials and set from that moment a path for the further investigation of such structures. Using the same material system, the same workgroup was able to observe, for the first time, the formation of UV-A radial modes at room temperature and whispering-gallery (WG) modes at $10 \mathrm{~K} .{ }^{201}$ The first UV-A microdisk pulsed-laser was achieved by Chang et al. ${ }^{202}$ demonstrating the first lasing WG features using a GaN microdisk as well as a decrease in the stimulated emission threshold by 10 times as compared with a GaN squared-shaped structure.

The development of the UV microdisk lasers increased in complexity, including studies on the optical pumping geometry, ${ }^{203}$ directional lasing emissions, ${ }^{196,204,205}$ electrical injections, ${ }^{196}$ foreign substrates, ${ }^{197-200,206-211}$ different crystal planes, ${ }^{208-210}$ quantum structures, ${ }^{200,206-211}$ and on new material systems, such as GaN/AIN and AlN/AlGaN. ${ }^{199,200,208-211}$ During this technological progress, smaller lasing thresholds and single-mode lasing were achieved, room temperature operation became the standard, and a UV-C lasing emission [center wavelength $\left(\lambda_{c}\right)=275 \mathrm{~nm}$ ] became a reality. ${ }^{200,211}$ Nonetheless, this progress comes with trade-off between different device parameters i.e., the first UV-C microdisk laser required a high pumping energy of $17,000 \mathrm{~kW} / \mathrm{cm}^{2}$, whereas the most recent UV-A single mode microdisk laser emitting at $379.25-\mathrm{nm}$ wavelength needs only $225 \mathrm{~kW} / \mathrm{cm}^{2}$. In Table 11 , we illustrate comprehensive historical progress of these III-nitrides-based microdisks UV emitters.

In addition to efforts mentioned above, Kneissl et al. ${ }^{196}$ demonstrated electrical injection of a spiral-shaped microdisk [Fig. 30(c)] on an InGaAlN laser heterostructure, obtaining a room-temperature directional radial/WG-multimode lasing at $399 \mathrm{~nm}$. So far, this is the only work of an electrically pumped microdisk laser with an emission wavelength in the UV spectral range below $400 \mathrm{~nm}$. Choi et al. ${ }^{197}$ demonstrated the first freestanding InGaN/GaN microdisks on Si by selectively dry-etching the substrate [Figs. 30(e) and 30(f)]. This enabled a bottom GaN/air interface instead of $\mathrm{GaN} / \mathrm{Si}$ interface, creating strain relaxation and improved material properties. Such a microdisk showed WG lasing emission at $\sim 365 \mathrm{~nm}$ at a $4.3-\mathrm{K}$ temperature and laid the demonstration of III-nitrides on Si platform. Next, Zhang et al. ${ }^{199}$ demonstrated a material system (AlN/AlGaN-on-Si) obtaining a single-mode lasing in the UV-B spectral range, being the only report of microdisks in such material system till now. Additionally, they study the structure by FDTD simulation of the WG resonant modes [Figs. 30(i) and 30(j)], obtaining mode matching between the experimental and the simulated results. Mexis et al. ${ }^{207}$ and Bürger et al. ${ }^{209}$ also demonstrated a mature computational approach to study the microdisk UV lasers.

One of the most recent report on microdisk lasers by Zhu et al. ${ }^{198}$ illustrates a concept of spontaneous formation of a sidewall grating [Figs. 30(g) and 30(h)], which enables the single-mode lasing at $379 \mathrm{~nm}$ of a GaN microdisk on Si substrate. Ultimately, it is important to highlight the work of Sellés et al., ${ }^{200,211}$ holding the record for the shortest wavelength III-nitrides microdisk laser of $275 \mathrm{~nm}$ [Fig. 30(k)]. This was achieved by integrating ultra-thin GaN/AIN MQW (0.7/5 nm) on $\mathrm{Si}$. As both GaN and AlN are binary compounds, the quality of the material is not compromised as compared with alloyed materials, e.g., ternary compounds, e.g., $\mathrm{AlGaN}$ and $\mathrm{InGaN}$.

\subsubsection{Nanopillars and nanowires}

Similarly to microdisks, nanopillars and NWs are structures with the potential to function as a lasing element in the UV spectral range. As seen in Table 12, the lasing of such structures has been achieved in a variety of pillar/wire dimensions and configurations. 
Alias et al.: Review of nanophotonics approaches using nanostructures...

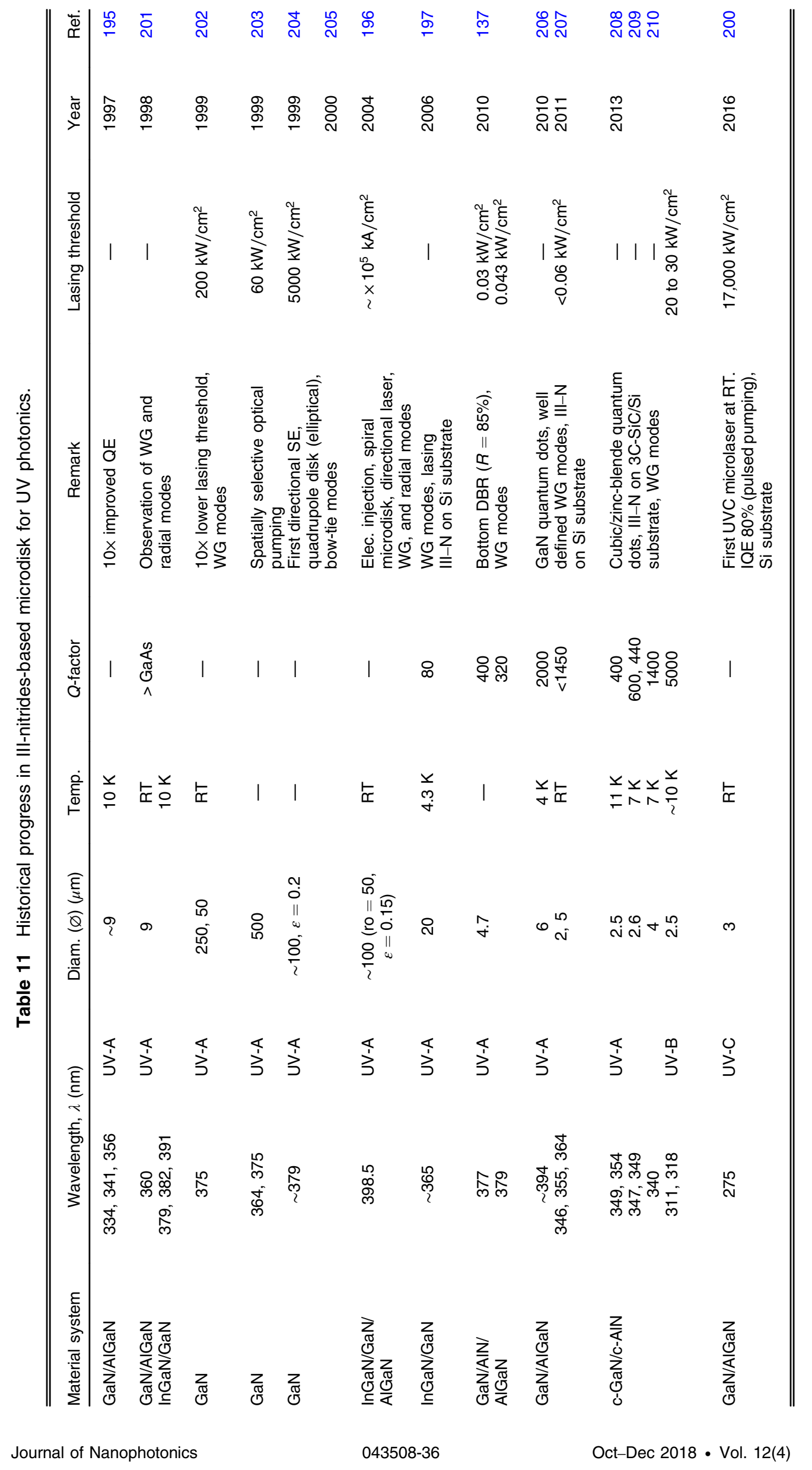


Alias et al.: Review of nanophotonics approaches using nanostructures...

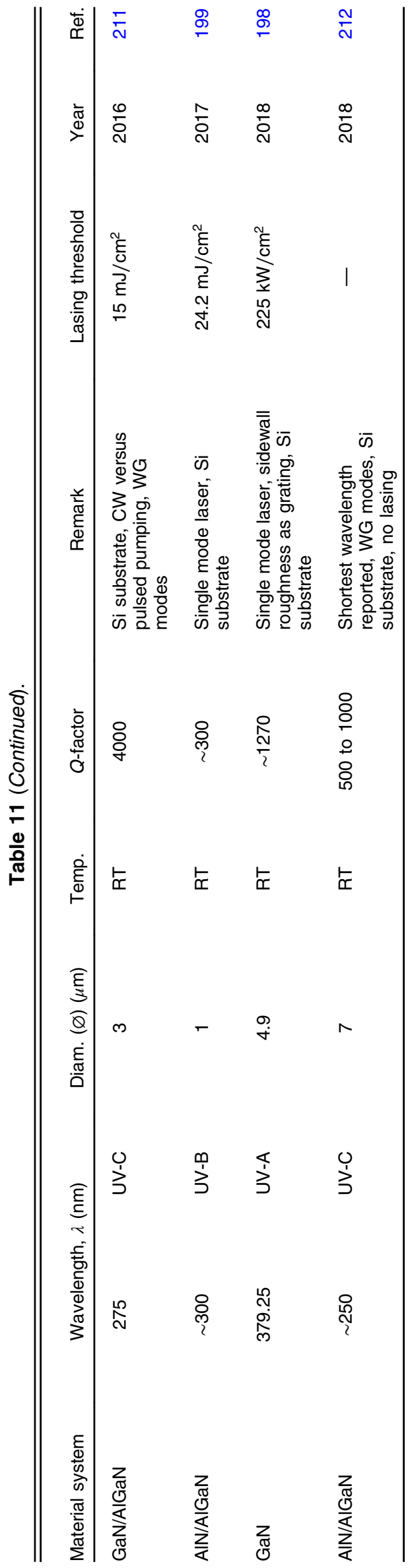


Alias et al.: Review of nanophotonics approaches using nanostructures...

Table 12 III-nitrides-based top-dow-etched nanopillars and nanowires for UV nanophotonics.

\begin{tabular}{|c|c|c|c|c|c|c|c|c|c|}
\hline \multirow{2}{*}{$\begin{array}{l}\begin{array}{l}\text { Material } \\
\text { system }\end{array} \\
\text { AllnGaN/ } \\
\text { AlGaN }\end{array}$} & \multicolumn{2}{|c|}{$\begin{array}{l}\text { Wavelength } \\
(\mathrm{nm})\end{array}$} & \multirow{2}{*}{$\begin{array}{l}\text { Diameter, } \\
\varnothing(\mu \mathrm{m}) \\
0.50\end{array}$} & \multirow{2}{*}{$\begin{array}{c}\text { Length, } \\
\frac{L(\mu \mathrm{m})}{1.00}\end{array}$} & Temp. & \multirow{2}{*}{$\begin{array}{l}\text { Remark } \\
\text { III-N bottom DBR, } \\
\mathrm{SiO}_{2} / \mathrm{HfO}_{2} \text { top DBR, } \\
Q=160\end{array}$} & \multirow{2}{*}{$\begin{array}{c}\begin{array}{c}\text { Lasing } \\
\text { threshold }\end{array} \\
520 \mathrm{~kW} / \mathrm{cm}^{2}\end{array}$} & \multirow{2}{*}{$\frac{\text { Year }}{2010}$} & \multirow{2}{*}{$\frac{\text { Ref. }}{213}$} \\
\hline & 344 & UV-A & & & RT I & & & & \\
\hline $\begin{array}{l}\text { InGaN/ } \\
\text { GaN }\end{array}$ & 373 & UV-A & 0.98 & 0.75 & $\mathrm{RT}$ & $\begin{array}{l}\text { Al-coated, single } \\
\text { WG mode lasing, } \\
Q=150\end{array}$ & $420 \mathrm{~kW} / \mathrm{cm}^{2}$ & 2012 & 214 \\
\hline $\mathrm{GaN}$ & 371 & UV-A & 0.14 & 4.70 & RT & $\begin{array}{l}\text { Freestanding NW on } \\
\text { SiN film substrate, } \\
\text { FP single mode } \\
\text { lasing }\end{array}$ & $231 \mathrm{~kW} / \mathrm{cm}^{2}$ & 2012 & 215 \\
\hline $\mathrm{GaN}$ & 370 & UV-A & $\begin{array}{l}0.68 \\
0.72\end{array}$ & $\begin{array}{l}7.60 \\
8.00\end{array}$ & - & $\begin{array}{l}\text { Single mode lasing } \\
\text { by Vernier effect } \\
\text { coupled cavity }\end{array}$ & 874 kW/cm² & 2012 & 216 \\
\hline $\mathrm{GaN}$ & 369 & UV-A & 0.35 & 5.30 & -5 & $\begin{array}{l}\text { Single mode lasing } \\
\text { by Au/SiN film } \\
\text { substrate mode } \\
\text { losses }\end{array}$ & $276 \mathrm{~kW} / \mathrm{cm}^{2}$ & 2012 & 217 \\
\hline $\mathrm{GaN}$ & $\begin{array}{c}363,364 \\
365\end{array}$ & UV-A & 0.30 & 1.20 & RT & $\begin{array}{l}\text { Al/SiN-coated, WG- } \\
\text { SP and cavity } \\
\text { modes, } Q \sim 120\end{array}$ & $5.2 \mathrm{~mJ} / \mathrm{cm}^{2}$ & 2013 & 218 \\
\hline $\mathrm{GaN}$ & 369 & UV-A & $\sim 0.20$ & 5.00 & -5 & $\begin{array}{l}\text { Single mode by } \\
\text { external SiN } 90 \mathrm{~nm} \\
\text { DFG }\end{array}$ & $\sim 300 \mathrm{~kW} / \mathrm{cm}^{2}$ & 2014 & 219 \\
\hline
\end{tabular}

A team composed of $\mathrm{Li}, \mathrm{Xu}$, Wright et al. utilizing a combination of dry-etching and anisotropic wet etching processes fabricated a set of GaN NWs [Figs. 31(c) and 31(d)]. ${ }^{215-217,219}$ These NWs were then isolated and used in several configurations to demonstrate UV-A lasing. First, a single NW $(\varnothing=0.14 \mu \mathrm{m}, L=4.7 \mu \mathrm{m})$ was investigated under optical pumping at room temperature. After the pumping threshold, a single-mode lasing at 371-nm wavelength was emitted from the longitudinal facets produced by the Fabry-Perot (FP) oscillations along the wire. Moreover, by setting two discrete GaN NWs one besides the other, a coupled cavity was created with single-mode lasing at $\lambda_{c}=370 \mathrm{~nm}$ [Figs. 31(a), 31(b), and 31(e)]. The lasing emission from such a coupled cavity is associated with the Vernier effect, i.e., suppression of resonant modes due to destructive interference. ${ }^{220}$

Due to the nature of this coupling effect, the lasing threshold was increased to $874 \mathrm{~kW} / \mathrm{cm}^{2}$; this is about three times higher than the lasing threshold of a single NW. Similarly, a single mode lasing in a discrete $\mathrm{GaN} \mathrm{NW}(\varnothing=0.35 \mu \mathrm{m}, L=5.3 \mu \mathrm{m})$ was achieved by a method of mode-selection based on the UV ohmic loss of the GaN-NW/Au interface. By modeling and calculating the propagation loss of different resonant modes within the NW cavity in contact with the metal, a specific mode (the one with less exposure to the $\mathrm{NW} /$ metal interface) is found to experience a loss significantly lower than any other optical mode and to be low enough to be overcome by the modal gain. It was quantified that using this method, the lasing threshold increases only by $13 \%$, reporting a threshold value of $276 \mathrm{~kW} / \mathrm{cm}^{2}$. Ultimately, a GaN NW $(\varnothing=0.2 \mu \mathrm{m}, L=5.0 \mu \mathrm{m})$ of the same nature was characterized by optical pumping while lying on a SiN NGs. It was observed that when the NW cavity is perpendicular to the grating pitch, the substrate grating works as a distributed feedback grating (DFG) enabling the single-mode lasing of the NW at a threshold power density of $\sim 300 \mathrm{~kW} / \mathrm{cm}^{2}$. It is important to highlight that the diameter and length of the GaN NWs are critical parameters toward single mode emission.

The reports by $\mathrm{Li}$ et al. ${ }^{214}$ and $\mathrm{Hsu}$ et al. ${ }^{218}$ demonstrated the use of $\mathrm{Al}$ thin-film coating on nanopillars [Figs. 31(f) and 31(g)], with the goal of providing UV optical confinement in the cavity 

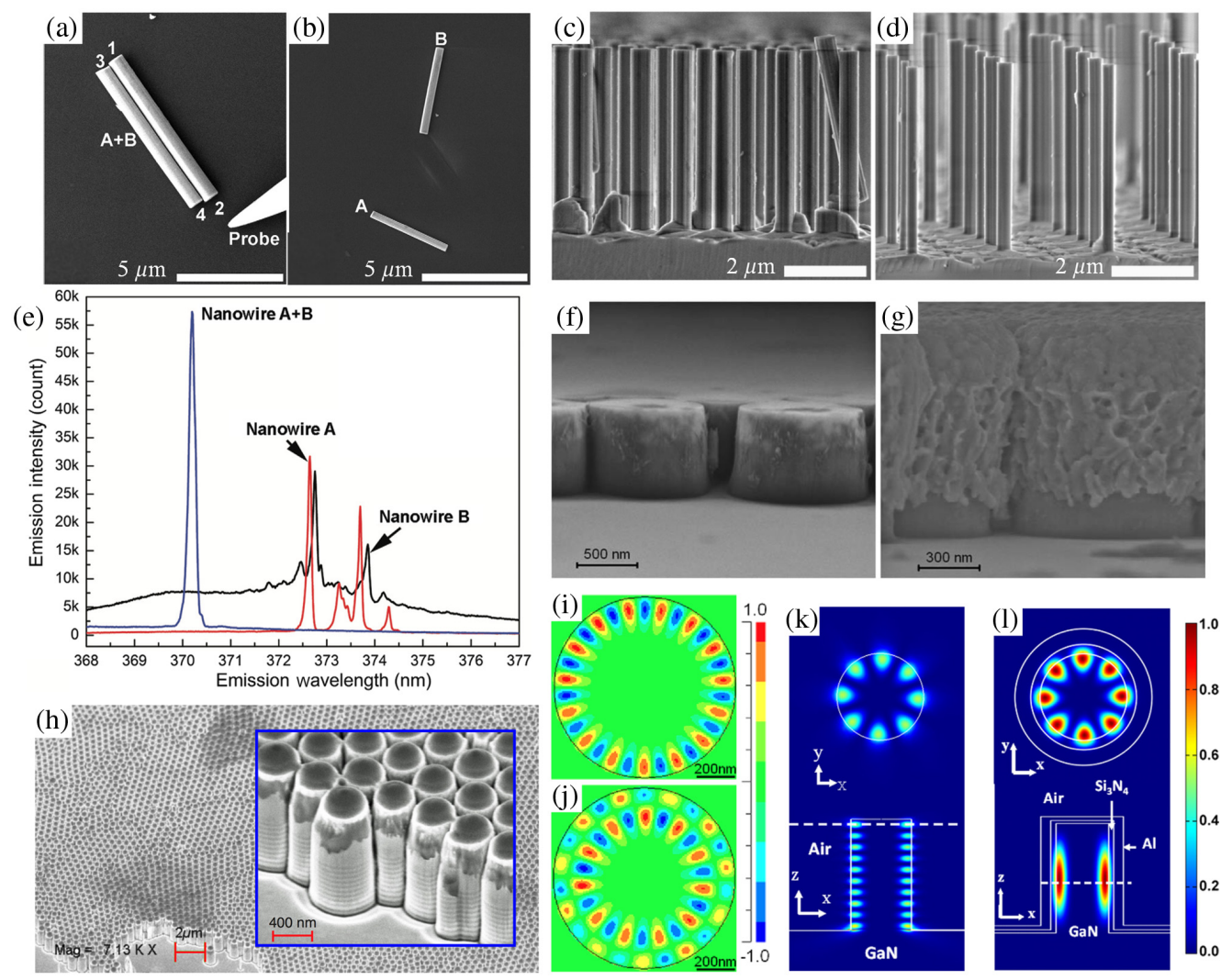

Fig. 31 Development of nanopillars and NWs as UV-light lasing structures. (a) GaN NW pair (1, 2, 3 , and 4 indicate the facets) formed by NW A and NW B as seen in (b), with their corresponding lasing spectra on (e). Reproduced with permission from Ref. 216. (c) and (d) are dry-etched GaN NWs after 6 and $9 \mathrm{~h}$ of additional wet etching. Reproduced with permission from Ref. 215. (f) An array of InGaN/GaN nanopillars before and after (g) Al metal coating. Reproduced with permission from Ref. 214. (h) SEM image of vertical-emitting UV lasing nanopillars with the bottom and upper DBR, and the inset shows a zoom-in SEM image. Reproduced with permission from Ref. 213. (i) and (j) The FDTD-simulated electric field of the WG modes in a micropillar, showing the different radius dependence for light at 365 and $373 \mathrm{~nm}$, respectively. Reproduced with permission from Ref. 214. (k) and (I) The calculated electric field distribution of a nanopillar without and with a metal mirror (Al/SiN) coating. Reproduced with permission from Ref. 218.

due to the high UV reflectivity of Al film. In Ref. 214, a WG-based single mode lasing at 373-nm wavelength is achieved at an optical pumping power density of $420 \mathrm{~kW} / \mathrm{cm}^{2}$. The WG nature of the lasing mode is verified through the peripheral far-field emission measurements around the set of nanopillars, relying on the fact that the single-mode WG lasing must be directional. Additionally, FDTD simulations were performed to understand the origin of the resonant peak wavelength. The emission spectra showed two resonant wavelengths for the nanopillars $\left(\lambda_{c 1}=365 \mathrm{~nm}, \lambda_{c 2}=373 \mathrm{~nm}\right)$. The emission of the peak at $365 \mathrm{~nm}$ belongs to the firstorder WG, which is exposed to the walls of the nanopillar being prone to losses [Fig. 31(i)]; whereas, the mode corresponding to $\lambda_{c 2}=373 \mathrm{~nm}$ is a higher order WG mode and therefore it remains within the nanopillar avoiding the losses encountered by the first-order mode [Fig. 31(j)]. Hsu et al. ${ }^{218}$ studied numerically and experimentally the role of the metal coating in maintaining the WG modes confined within the cavity. As a result of this approach, a twofold cavity confinement enhancement is achieved as compared with the nanopillars without the metal coating [Figs. 31(k) and 31(1)]. Additionally, they demonstrated that nanopillars with sidewall angles nearer to $90 \mathrm{deg}$ are preferred to improve the cavity quality. Chen et al. ${ }^{213}$ suggested another approach by utilizing a AlInGaN-based MQW active region existing between a bottom $\mathrm{AlGaN}$-based DBR and a top $\mathrm{HfO}_{2} / \mathrm{SiO}_{2}$-based DBR to create an enhanced vertical-cavity [Fig. 31(h)]. 


\subsubsection{Other unique micro- and nanostructures}

Additionally, to what has been discussed earlier, we have found a set of unique structures that can significantly impact the development of UV photonics. Zeng et al. ${ }^{221}$ demonstrated the first InGaN/GaN-based microring resonator in UV-A regime. Due to the shape of these structures, radial modes are not allowed, and only WG resonant modes can be produced. This fact represents an advantage of the ring-structures when developing WG resonators. Following the microring, Wang et al. ${ }^{222}$ demonstrated a GaN nanoring [Fig. 32(a)] enhanced by a coating of thin $\mathrm{Al}$ $(50 \mathrm{~nm})$. In this report, experimental and computational characterizations agree that the device presented hybrid WG-SP lasing modes $\left(\lambda_{c} \sim 365 \mathrm{~nm}\right)$ created from the combination of the ringshaped semiconductor and the evanescent fields on the semiconductor/metal interface. Although this effect is not desired for single-mode lasing devices, the nature of the plasmonic resonances opens up the possibility of using this kind of nanorings for sensing at the nanoscale. ${ }^{227}$ The same research group fabricated a $\mathrm{GaN}$ nanostripe, coated with $20-\mathrm{nm} \mathrm{SiO}_{2}$ and $60 \mathrm{~nm} \mathrm{Al}$ [Fig. 32(b)]. ${ }^{223}$ Here, they observed lasing at $\lambda_{c}=370 \mathrm{~nm}$ under a relatively low optical power density threshold of $0.042 \mathrm{~kW} / \mathrm{cm}^{2}$, at room temperature. Due to the waveguide-like shape of the nanostripe, and because of the highly reflective internal walls (Al), lateral confinement exists, and an FP cavity is formed in the transversal plane.

An innovative approach is presented by Wang et al. demonstrating high $Q$ (>6000) WGmode lasing at room temperature in a GaN micropyramid $\left(\lambda_{c}=367.2 \mathrm{~nm}\right.$, lasing threshold $=400$ to $\left.500 \mathrm{~kW} / \mathrm{cm}^{2}\right){ }^{225}$ These GaN pyramids are created by $\mathrm{KOH}$ wet etching of GaN, previously transferred onto a $\mathrm{Ni}-\mathrm{Ag}-\mathrm{Pt}-\mathrm{Au} / \mathrm{Cu}$ substrate. This nonconventional method allows the formation of high-quality GaN micropyramids standing on a metallic reflector [Fig. 32(d)]. Because of this configuration, the microcavity is capable of sustain "quasi-WG" lasing modes fed by the pyramid faces and the bottom mirror. Another unique microstructure was reported by Zhang et al. ${ }^{224}$ including a very comprehensive study of the material properties, the optical cavity, the power injection, as well as the carrier dynamics. Mushroom-like structures (hemispherical microcavities) [Fig. 32(c)] were achieved in an InGaN/GaN-on-Si system showing WG lasing modes with the strongest lasing peak at $\lambda_{c}=381 \mathrm{~nm}$. One benefit of such hemispherical structure is the elimination of parallel facets (like those in microdisks), ideally
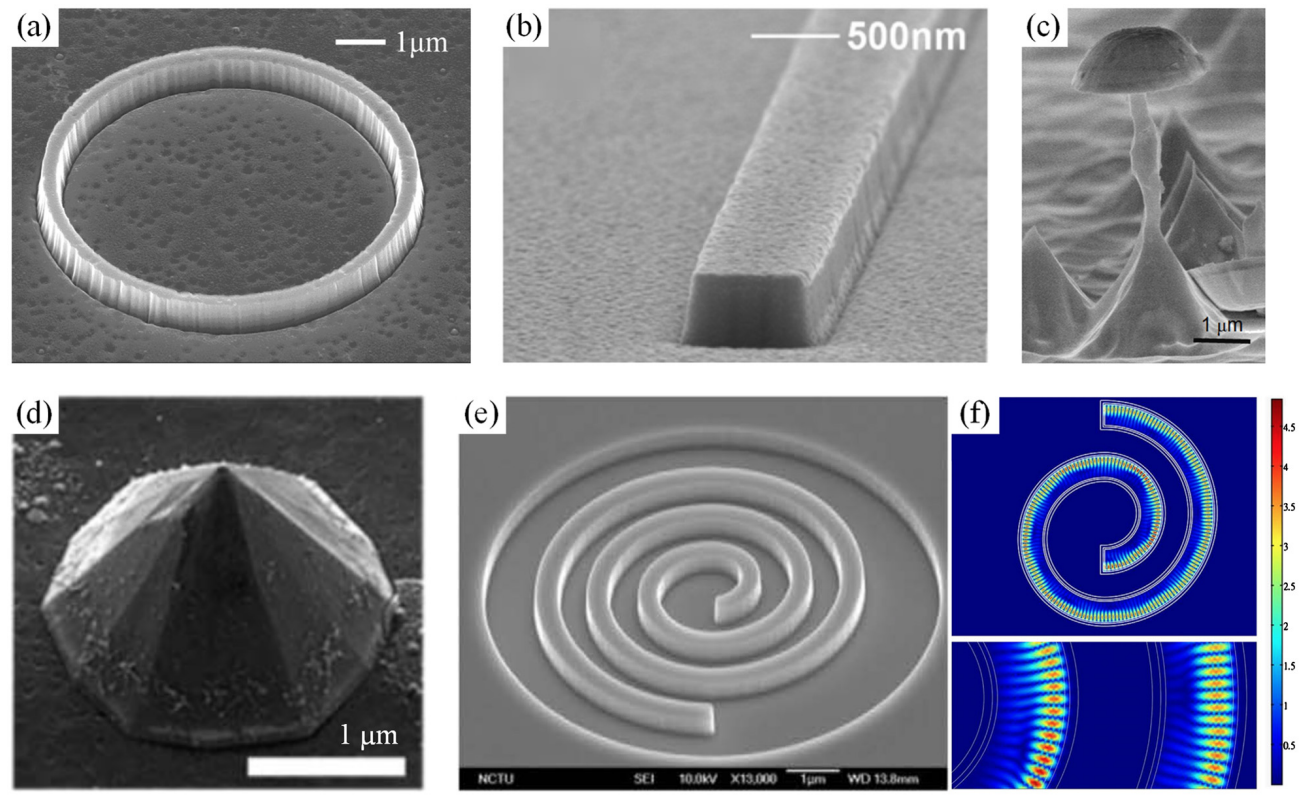

Fig. 32 Set of unique III-nitrides-based UV-lasing micro and nanostructures. (a) Metal-coated nanoring. Reproduced with permission from Ref. 222. (b) Metal-coated nanostripe. Reproduced with permission from Ref. 223. (c) Hemispherical microcavity on Si post. Reproduced with permission from Ref. 224. (d) Micropyramid on a metal substrate. Reproduced with permission from Ref. 225. (e) Metal-coated nanospiral with calculated electric field distribution (f). Reproduced with permission from Ref. 226. 
removing any possibility of observing FP oscillations, creating pure WG/ radial modes. The last structure discussed is the nanospiral created by Liao et al. [Fig. 32(e)]. ${ }^{226}$ This structure is made of $\mathrm{GaN}$ coated with $30-\mathrm{nm} \mathrm{SiN}$ and 50-nm Al thin film. Circularly polarized UV-A lasing at $\lambda_{c}=363.5 \mathrm{~nm}$ was demonstrated at a low lasing threshold of 0.017 and $0.016 \mathrm{~kW} / \mathrm{cm}^{2}$, respectively, for left and right circular polarizations. Similar to the previous structures coated with Al, the metal significantly enhances the transversal cavity and light confinement [Fig. 32(f)]; however, in this spiral, the role of the longitudinal traveling modes along the waveguide-like structure is critical to creating polarization rotation. This polarization rotation could be generated by the minor changes of curvature along the GaN-Al interface, impinging a small phase change to the lateral confined modes. Moreover, these observations open opportunities for further research and development of nanosources of circularly polarized UV light. To the present day, this nanoscale circularly polarized UV laser, represent by itself a breakthrough in UV nanophotonics.

\subsection{Surface Plasmons}

SPs are the collective oscillations of free electrons at a metal-dielectric or metal-semiconductor interface, where the real part of the dielectric function changes its sign. ${ }^{228}$ Plasmonics attributes optical technology of coupling SPs to electromagnetic waves. This merged photonic-electronic phenomenon excites propagating SPPs on planar surfaces or localized SPs (LSPs) on nanoparticles. Both of the SPPs and LPSs manifest themselves as evanescent electromagnetic waves with a penetration depth of only a few tens of nanometers in metal, at optical frequencies. The dispersion properties of SPPs and LSPs are unique as they significantly enhance the incident electromagnetic field near the metallic structures. ${ }^{228,229}$

SPs can boost the EQE of UV light emitters through two ways, enhancing its LEE and IQE. ${ }^{230-233}$ In the first way, generated photons captured within the device are intentionally directed toward a metallic layer to excite SPPs on its surface. ${ }^{230}$ The condition of this process is that the plasmonic resonance wavelength of the metal matches the wavelength of the generated light. If the metal surface is rough enough to scatter SPPs as light out of the device, the LEE of the UV emitter can soar because of extracting the SP-induced magnified light out of the emitter. The second way dramatically increases the emitter IQE through the exciton-SP coupling. ${ }^{231,232}$ This way is contingent on placing a QW near a metallic plasmonic surface such that electronhole pairs are located within the near field of the metallic surface. In this scenario, some electronhole pairs recombine and directly transfer their energy to the electrons oscillations on the metal surface to produce SPs instead of photons. This new recombination path, along with the typical electron-hole recombination which generates photons, decreases the spontaneous emission lifetime, and thus enhances the emitter IQE. Here, we discuss the SPs approaches for UV-photonic devices, which include approaches of thin films, a patterned layer, metamaterial, and nanostructures.

\subsubsection{Thin plasmonic film}

Gao et al. followed the first way mentioned above to enhance the device performance using AlGaN-based UV-LED [Fig. 33(a)]. ${ }^{230}$ In this structure, a 5-nm-thick Al layer is deposited above the MQW structure. When electron-hole pairs recombine in AlGaN alloys, they generate photons with a dominant polarization parallel to the crystal axis $(E \| c)$. Consequently, as shown in Fig. 33(a), only weak light signal of polarization $E \perp c$ can be extracted through the escaping cone. The remaining light signals (entire $E \| c$ and part of $E \perp c$ polarization) are captured within the LED and propagate parallel to the Al layer. The $E \| c$ polarization one is a TM wave for the $\mathrm{Al}$ layer and can excite SPPs on its surface at 294-nm wavelength. Figures 33(b) and 33(c) show the atomic force microscopy (AFM) images for the top surfaces of the UV-LED and Al layer, respectively. Figure 33(d) compares the PL of the UV-LED without Al, after depositing the Al, and after oxidizing the Al. SP-enhancement of $217 \%$ is acquired for the PL at 294-nm wavelength.

Lin et al. ${ }^{231}$ presented an example for SP-enhanced IQE of UV emitter. A schematic of the AlGaN/GaN QW structure is shown in the inset of Fig. 33(a). The QW is fabricated using MBE and coated with $\mathrm{Ag}, \mathrm{Au}$, or $\mathrm{Al}$ to excite SPPs on their surfaces. The top AlGaN layer is thin enough to raise the spontaneous emission decay rate of the carriers through direct transfer of the 

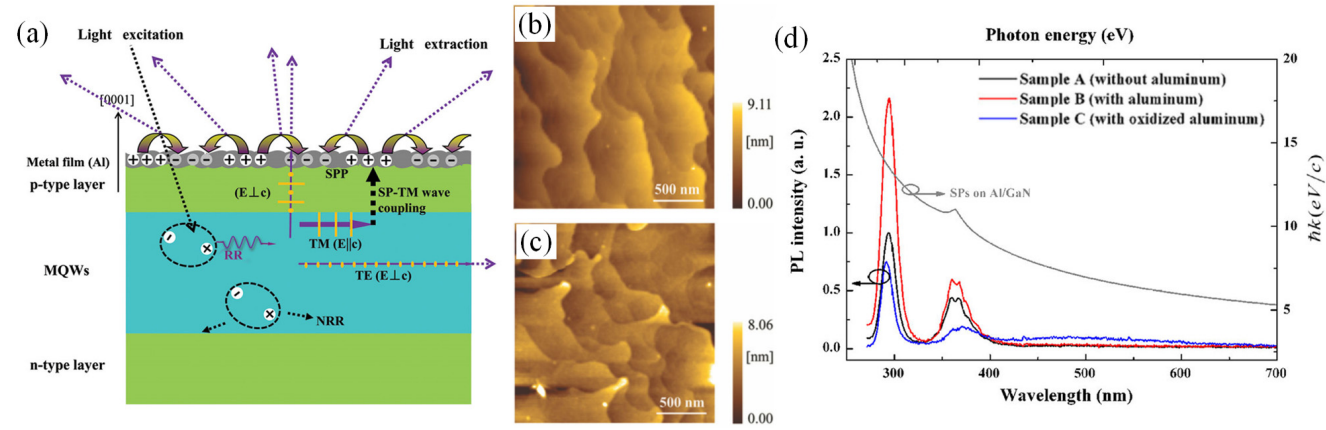

Fig. 33 (a) Schematic of the SP-enhanced AIGaN-based UV-LED. AFM images for the top surfaces of the (b) UV-LED and (c) Al layer. (d) PL spectra of the structures. Reproduced with permission from Ref. 230.
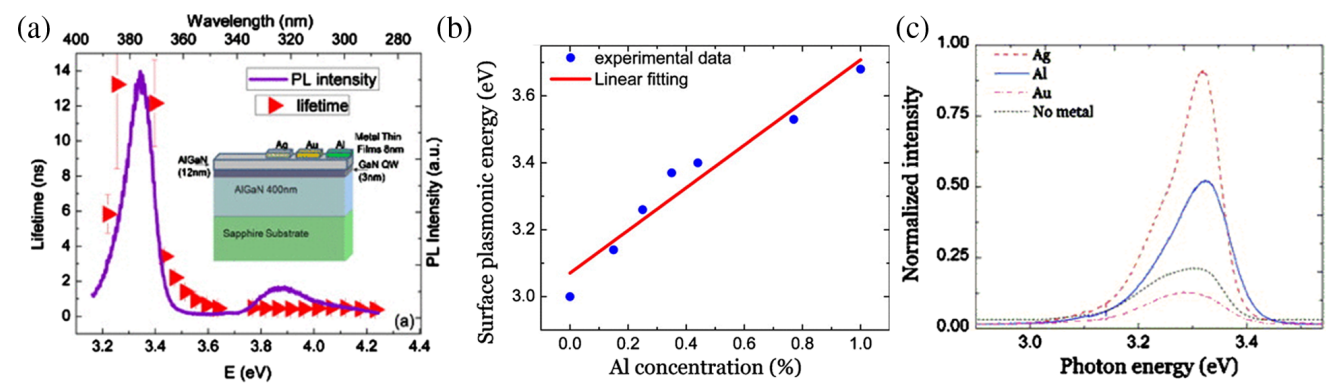

Fig. 34 (a) PL spectrum and carrier lifetime with a schematic of AIGaN/GaN QW shown in the inset. (b) Change of the SP energy of the Ag layer with Al content of the AIGaN barrier layer. (c) Comparing the PL of the metal-free AIGaN/GaN QW and with other metals. Reproduced with permission from Ref. 231.

electron-hole energies to the electrons oscillations on the metals surfaces. Before metal deposition onto the device, the PL intensity and lifetime of the carriers were measured at $10 \mathrm{~K}$ [Fig. 34(a)]. Interestingly, this research group discovers the ability to tune the plasmonic resonance wavelength of $\mathrm{Ag}$ from the visible to $\mathrm{UV}$ region by tuning the $\mathrm{Al}$ content of the $\mathrm{AlGaN}$ barrier [Fig. 34(b)]. When the Al content within the barrier layer is $~ 23 \%$, the Ag plasmonic resonance matches the emission wavelength of the GaN QW. In Fig. 34(c), the SPenhancement of the PL emission from the GaN QW is compared for three different metals. A threefold increase in the UV-light emission is obtained when using Ag as a plasmonic layer.

\subsubsection{Patterned plasmonic layer}

Cho et al. $^{232}$ reported an SP-enhanced IQE of AlGaN-based UV-LED using a patterned plasmonic layer. The LED structure is schematically shown in Fig. 35(a). To provide an exciton-SP

(a)

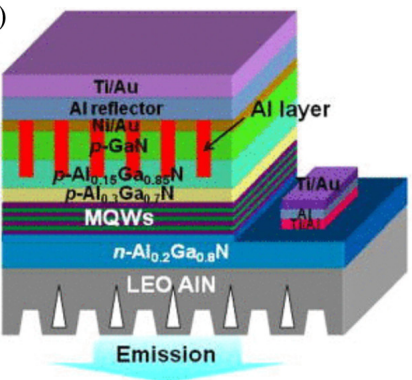

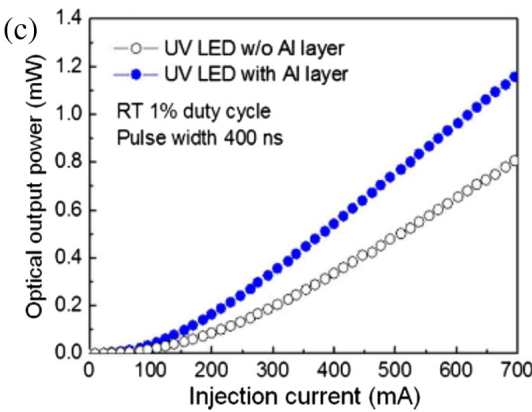

Fig. 35 (a) Schematic of the SP-enhanced UV-LED. (b) Top-view SEM image after Al deposition. (c) The output power as a function of the injection current for the LEDs with and without Al layer. Reproduced with permission from Ref. 232. 
coupling, the top most $p$-AlGaN and $p$-GaN layers are patterned with vertical holes via photolithography and dry-etching. Afterward, Al pillars are deposited inside the holes to be in the very close vicinity to the MQW, as shown in Fig. 35(a). Figure 35(b) shows the top-view SEM image after $\mathrm{Al}$ deposition. Then, $\mathrm{Al}$ and Ti/Au layer were deposited on top of the device to work as reflectors, and thus light is extracted from the backside, as shown in Fig. 35(a). The output power of the SP-enhanced LED is 45\% higher than that of the control LED [Fig. 35(c)]. This enhancement is attributed to the increase in the spontaneous emission decay rate by the exciton-SP coupling.

\subsubsection{Hyperbolic metamaterial}

An alternative approach is introduced by Shen et al. ${ }^{233}$ to use resonant SPs modes excited within hyperbolic metamaterial (HMM) to enhance simultaneously the IQE and LEE of AlGaN-based UV-LED. In comparison with the typical SPs structures, HMM is made of multiple metal-dielectric interfaces. Excitons from MQW close to the HMM can excite its resonant SPs modes, which increase their spontaneous emission decay rate and the IQE. A significant PL enhancement by $520 \%$ is achieved for HMM-based LED in comparison with the control LED.

\subsubsection{Plasmonic nanostructures}

Son et al. investigated the influence of plasmonic nanostructures on the performance of AlGaN/ GaN LED. ${ }^{234}$ The plasmonic nanostructure used in these LEDs consists of $\mathrm{Al}$ nanorings, which are assembled directly above the MQW as shown in Fig. 36(a) to allow direct exciton-SP coupling. The fabrication steps of the Al nanorings are summarized in Fig. 36(b). The diameter of the nanorings can be tuned through the diameter of the silica nanospheres until their plasmonic resonance wavelength matches the emission wavelength of the LED. Figure 36(c) shows the AFM of the LED, where the Al nanorings were fabricated using 300-nm diameter silica nanospheres. The PL spectra [Fig. 36(d)] show various enhancements due to the IQE improvement from the exciton-SP coupling. Recently, Huang et al. ${ }^{235}$ used LSPs excitation from Al nanoparticles to improve the LEE of a UV-LED. Using the oblique angle deposition method, Al nanoparticles were deposited at the surface of the LED. At 60-deg deposition angle, maximum PL enhancement (329\%) is recorded at 282-nm emission wavelength. This enhancement occurs because when the photons excite LSPs on the surfaces of the Al nanoparticles, these LSPs recombine and generate photons with stronger intensities. For the non-LED device, Shetty et al. ${ }^{236}$ investigated the ability of plasmonic nanostructures to improve the performance of
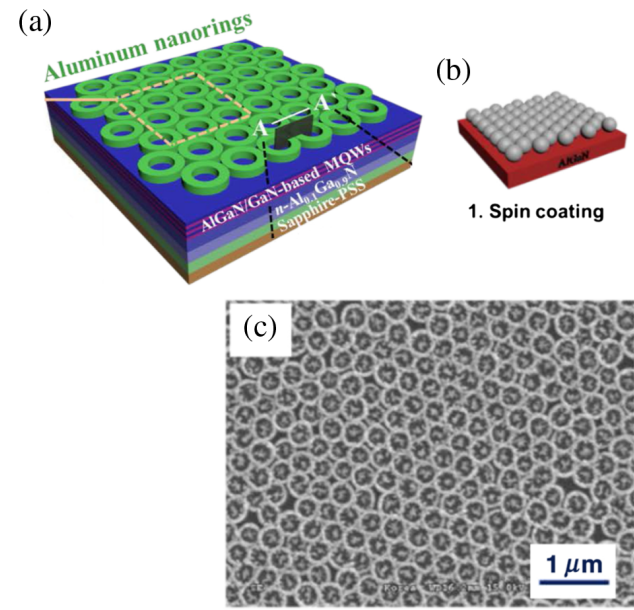

(b)

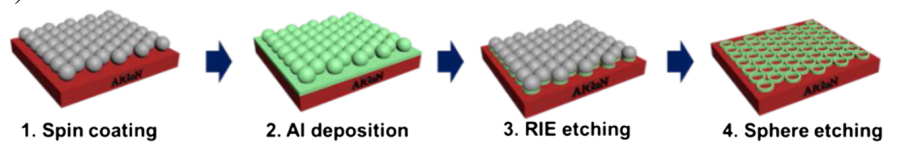

(d)

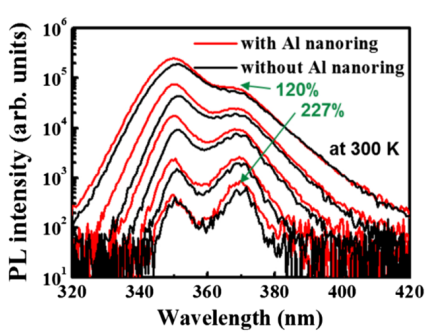

Fig. 36 (a) Schematic of the AIGaN/GaN MQW with Al nanorings. (b) Steps of Al nanorings deposition above the MQW. (c) AFM image for the nanorings above the MQW when using 300-nm silica nanospheres. (d) PL enhancement when measured at 300-K temperature. Reproduced with permission from Ref. 234. 
Alias et al.: Review of nanophotonics approaches using nanostructures...

Table 13 Summary of SP-enhanced UV-photonic devices.

\begin{tabular}{|c|c|c|c|c|c|}
\hline Technique & Device type & Device structure & $\begin{array}{l}\text { Device } \\
\text { characteristics }\end{array}$ & Enhancement & Ref. \\
\hline \multirow[t]{2}{*}{$\begin{array}{l}\text { Thin plasmonic } \\
\text { film }\end{array}$} & UVC-LED & Sapphire/AIGaN/GaN & $\begin{array}{l}\text { Peak emission } \\
\text { wavelength- } \\
294 \mathrm{~nm}\end{array}$ & $217 \%(P L)$ & 230 \\
\hline & UVA-LED & Sapphire/AIGaN/GaN/AIGaN & $\begin{array}{l}\text { Peak emission } \\
\text { wavelength- } \\
372 \mathrm{~nm}\end{array}$ & $200 \%(P L)$ & 231 \\
\hline $\begin{array}{l}\text { Patterned } \\
\text { plasmonic } \\
\text { layer }\end{array}$ & UVA-LED & $\begin{array}{l}\text { Si/AIN/n-AIGaN/AIGaN- } \\
\mathrm{MQW} / p-A I G a N / p-G a N\end{array}$ & $\begin{array}{l}\text { Peak emission } \\
\text { wavelength- } \\
346 \mathrm{~nm} \\
I_{\mathrm{th}}-\sim 100 \mathrm{~mA} \\
P_{\mathrm{o}}-\sim 1.2 \mathrm{~mW} \text { at } \\
I=700 \mathrm{~mA}\end{array}$ & $45 \%(P L)$ & 232 \\
\hline HMM & UVB-LED & Sapphire/AIGaN/HMM/AI & $\begin{array}{l}\text { Peak emission } \\
\text { wavelength- } \\
318 \mathrm{~nm}\end{array}$ & $520 \%$ (EQE) & 233 \\
\hline \multirow[t]{3}{*}{$\begin{array}{l}\text { Plasmonic } \\
\text { nanostructures }\end{array}$} & UVC-LED & $\begin{array}{l}\text { Sapphire/AIN/Al }{ }_{0.1} \mathrm{Ga}_{0.9} \mathrm{~N} / n- \\
\mathrm{Al}_{0.1} \mathrm{Ga}_{0.9} \mathrm{~N} / \mathrm{MQW}\end{array}$ & $\begin{array}{l}\text { Peak emission } \\
\text { wavelength- } \\
266 \mathrm{~nm} \\
\text { Pump power } 0.03 \text { to } \\
8 \mathrm{~mW}\end{array}$ & $227 \%(P L)$ & 234 \\
\hline & UVB-LED & $\begin{array}{l}\text { Sapphire/AIN/n-AIGaN/MQW/ } \\
p \text {-AlGaN/p-GaN }\end{array}$ & $\begin{array}{l}\text { Peak emission } \\
\text { wavelength- } \\
282 \mathrm{~nm}\end{array}$ & $329 \%(P L)$ & 235 \\
\hline & $\begin{array}{l}\text { UVA-UVB- } \\
\text { PD }\end{array}$ & Metal-GaN-metal & $\begin{array}{l}\text { Absorption } \\
\text { wavelength-300 to } \\
400 \mathrm{~nm}\end{array}$ & Photocurrent & 236 \\
\hline
\end{tabular}

UV-PD. Incident UV light on the PD excites LSPs on the surface of Al nanostructures. The magnified fields near the metallic structures are then scattered to increase the PD photocurrent.

In Table 13, we summarize the different trials of enhancing UV-photonic devices using SPs. The table provides a comparison regarding the fabrication technique, device type, structure, characteristics, and the enhancement percentage.

\section{Conclusions and Outlook}

The continuously increasing development in UV-photonic devices has mainly resulted from the intensive research on nanophotonics and the advanced nanofabrication approaches applied to realize nanostructures. In this review, we have given a comprehensive description of nanophotonics implementations using nanostructured interfaces and nanofabrication approaches for the group III-nitride semiconductors to realize efficient UV photonic devices.

The NWs-based technology has recently been pursued due to the unique properties of NWs, e.g., high crystalline quality with low defect density and large surface-to-volume ratio. We have reviewed the most significant ways to grow high-quality NWs for UV applications. Lithographybased SAG, VLS growth, and the catalyst-free self-assisted growth schemes have been presented. The key element to improve the performance of UV-photonic devices is to reduce the TDDs and to solve the cracking issue of AlN and AlGaN films, which stems from the tensile strain beyond the critical layer thickness. Therefore, to enhance the material quality of epilayers grown on nonnative substrates, different ELOG schemes have been suggested and successfully employed to enhance the performance of UV devices. We show that research on GaN-based QDs and QDisks is very promising to develop highly efficient UV-photonic devices. Such devices are already outperforming their counterparts regarding material quality, IQE, LEE, responsivity, 
sensitivity, and the tunability of the wavelength. However, the optical output power of such devices still needs to be improved.

The performance of UV-photonic devices can be also enhanced by utilizing surface roughening technologies, e.g., surface texturing and surface patterning. These technologies have shown a high impact on the LEE and device characteristics due to their abilities in increasing the extraction of photons, controlling the lateral waveguiding emission, and in improving the transparent $p$-contact layer. Further advances in lithography techniques will facilitate the roughening fabrication process and ensure high-resolution structure/surface features. We have also reviewed different shapes of PSSs, e.g., stripe-shaped, volcano-shaped, and groove-shaped, fabricated by the combination of a lithography method and either an additive technique (depositing a thin film on the substrate surface) or a subtractive technique (etching the substrate surface). We have shown that the utilization of PSSs is very effective to enhance the performance of the lightemitting UV devices in terms of decreasing the TDDs and increasing the LEE.

LEE, IQE, and EQE of the UV-photonic devices can also be improved using PhCs structures. In the case of DBRs which is a 1-D PhCs, we have reviewed the recent development of dielectric, epitaxial nitrides, and nitride/porous DBRs. Achieving a truly crack-free, broad stopband, and electrically conducting DBRs for the UV spectral range remains the main challenge. Several achievements on the usage of 2-D and 3-D PhCs on UV-LEDs have been shown. Furthermore, several types of NGs were reviewed, e.g., SWGs in the form of WGPs and 1D/2-D grating patterns, and a DBR grating. Continuous advancement in the field of direct laser writing, for example, will ease and speed up true 3-D structure prototyping and testing, allowing for greater precision and smaller nanofeatures to be fabricated.

For the case of micro- and nanostructures, such as microdisk, nanopillars, and NWs, we have reviewed the reported structures and devices based on III-nitrides operating in the UV spectral range. We expect that the continuously improving quality of III-nitride materials, as well as cross-conceptual approaches, will further enhance the performance of UV lasing devices for future applications. We have discussed different plasmonic-based approaches used to improve the performance of UV devices. We have shown that Al-based plasmonic structures have been primarily used to enhance the EQE of UV emitters significantly. One major hurdle of using SP-enhanced UV devices is Al oxidation, which inhibits their feasibility. Therefore, it is necessary to discover other metallic materials that have plasmonic resonance within the UV range and do not oxidize in air. Further, we recommend trying to incorporate plasmonic structures, such as Al nanoparticles, within the active region, which would significantly increase IQE of UV emitters.

\section{Acknowledgments}

The authors acknowledge funding support from King Abdulaziz City for Science and Technology (KACST) Technology Innovation Center (TIC) for Solid-State Lighting (KACST TIC R2-FP-008), and KAUST baseline funding (BAS/1/1614-01-01).

\section{References}

1. P. Mukish and P. Boulay, "UV LEDs-technology, manufacturing and application trends," 2016, https://www.i-micronews.com/led-report/product/uv-leds-technologymanufacturing-and-application-trends.html.

2. M. Kneissl and J. Rass, III-Nitride Ultraviolet Emitters, Vol. 227, Springer Series in Materials Science, Springer, Cham, Switzerland (2016).

3. J. Y. Tsao et al., "Ultrawide-bandgap semiconductors: research opportunities and challenges," Adv. Electron. Mater. 4(1), 1600501 (2018).

4. L. Sang, M. Liao, and M. Sumiya, "A comprehensive review of semiconductor ultraviolet photodetectors: from thin film to one-dimensional nanostructures," Sensors 13(8), 10482 10518 (2013).

5. S. C. Jain et al., "III-nitrides: growth, characterization, and properties," J. Appl. Phys. 87(3), 965-1006 (2000). 
6. M. A. Khan et al., "III-nitride UV devices," Jpn. J. Appl. Phys. 44(10), 7191-7206 (2005).

7. M. Kneissl et al., "Advances in group III-nitride based deep UV light-emitting diode technology," Semicond. Sci. Technol. 26(1), 014036 (2011).

8. Y. Taniyasu and M. Kasu, "Polarization property of deep-ultraviolet light emission from C-plane AlN/GaN short-period superlattices," Appl. Phys. Lett. 99(25), 251112 (2011).

9. Y. Tang et al., "Ultrahigh-speed GaN high-electron-mobility transistors with $\mathrm{f}_{\mathrm{T}} / \mathrm{f}_{\max }$ of 454/444 GHz," IEEE Electron Device Lett. 36(6), 549-551 (2015).

10. H. X. Jiang and J. Y. Lin, "Hexagonal boron nitride for deep ultraviolet photonic devices," Semicond. Sci. Technol. 29(8), 084003 (2014).

11. X. Li et al., "BAlN thin layers for deep UV applications," Phys. Status Solidi A 212(4), 745-750 (2015).

12. H. X. Jiang and J. Y. Lin, "Review-hexagonal boron nitride epilayers: growth, optical properties and device applications," ECS J. Solid State Sci. Technol. 6(2), Q3012-Q3021 (2017).

13. N. Izyumskaya et al., "Recent development of boron nitride towards electronic applications," Adv. Electron. Mater. 3(5), 1600485 (2017).

14. H. Hirayama et al., "Recent progress and future prospects of AlGaN-based high-efficiency deep-ultraviolet light-emitting diodes," Jpn. J. Appl. Phys. 53(10), 100209 (2014).

15. D. Li et al., "AlGaN photonics: recent advances in materials and ultraviolet devices," Adv. Opt. Photonics 10(1), 43-110 (2018).

16. J.-S. Park et al., "Review-group III-nitride-based ultraviolet light-emitting diodes: ways of increasing external quantum efficiency," ECS J. Solid State Sci. Technol. 6(4), Q42-Q52 (2017).

17. S. Zhao et al., "III-nitride nanowire optoelectronics," Prog. Quantum Electron. 44, 14-68 (2015).

18. N. Tansu et al., "Temperature analysis and characteristics of highly strained InGaAsGaAsP-GaAs $(\lambda>1.17 \mu \mathrm{m})$ quantum-well lasers," IEEE J. Quantum Electron. 38(6), 640-651 (2002).

19. A. Khan, K. Balakrishnan, and T. Katona, "Ultraviolet light-emitting diodes based on group three nitrides," Nat. Photonics 2(2), 77-84 (2008).

20. J. Li et al., "Optical and electrical properties of Mg-doped p-type $\mathrm{Al}_{\mathrm{x}} \mathrm{Ga}_{1-\mathrm{x}} \mathrm{N}$," Appl. Phys. Lett. 80(7), 1210-1212 (2002).

21. B. Mandl et al., "Au-free epitaxial growth of InAs nanowires," Nano Lett. 6(8), 1817-1821 (2006).

22. S. D. Hersee, X. Sun, and X. Wang, "The controlled growth of GaN nanowires," Nano Lett. 6(8), 1808-1811 (2006).

23. S. H. Oh et al., "Point defect configurations of supersaturated Au atoms inside Si nanowires," Nano Lett. 8(4), 1016-1019 (2008).

24. K. Kishino and S. Ishizawa, "Selective-area growth of GaN nanocolumns on $\mathrm{Si}(111)$ substrates for application to nanocolumn emitters with systematic analysis of dislocation filtering effect of nanocolumns," Nanotechnology 26(22), 225602 (2015).

25. R. Calarco et al., "Nucleation and growth of GaN nanowires on $\mathrm{Si}(111)$ performed by molecular beam epitaxy," Nano Lett. 7(8), 2248-2251 (2007).

26. P. Kumar et al., "Superstructure of self-aligned hexagonal GaN nanorods formed on nitrided Si(111) surface," Appl. Phys. Lett. 101(13), 131605 (2012).

27. K. Hestroffer et al., "In situ study of self-assembled GaN nanowires nucleation on Si(111) by plasma-assisted molecular beam epitaxy," Appl. Phys. Lett. 100(21), 212107 (2012).

28. M. Tangi et al., "Role of quantum-confined stark effect on bias dependent photoluminescence of N-polar GaN/InGaN multi-quantum disk amber light emitting diodes," J. Appl. Phys. 123(10), 105702 (2018).

29. M. Tangi et al., "Electron mobility of self-assembled and dislocation free InN nanorods grown on GaN nano wall network template," J. Appl. Phys. 119(20), 205701 (2016).

30. M. Tangi et al., "Bandgap measurements and the peculiar splitting of $\mathrm{E}_{2}{ }^{\mathrm{H}}$ phonon modes of $\mathrm{In}_{\mathrm{x}} \mathrm{Al}_{1-\mathrm{x}} \mathrm{N}$ nanowires grown by plasma assisted molecular beam epitaxy," J. Appl. Phys. 120(4), 045701 (2016). 
31. E. Calleja et al., "Growth, morphology, and structural properties of group-III-nitride nanocolumns and nanodisks," Phys. Status Solidi B 244(8), 2816-2837 (2007).

32. T. Gotschke et al., "Influence of the adatom diffusion on selective growth of $\mathrm{GaN}$ nanowire regular arrays," Appl. Phys. Lett. 98(10), 103102 (2011).

33. K. Choi, M. Arita, and Y. Arakawa, "Selective-area growth of thin GaN nanowires by MOCVD," J. Cryst. Growth 357(1), 58-61 (2012).

34. Y.-T. Lin, T.-W. Yeh, and P. D. Dapkus, "Mechanism of selective area growth of GaN nanorods by pulsed mode metalorganic chemical vapor deposition," Nanotechnology 23(46), 465601 (2012).

35. Y. T. Lin et al., "Catalyst-free GaN nanorods synthesized by selective area growth," Adv. Funct. Mater. 24(21), 3162-3171 (2014).

36. B. H. Le et al., "Controlled coalescence of AlGaN nanowire arrays: an architecture for nearly dislocation-free planar ultraviolet photonic device applications," Adv. Mater. 28, 8446-8454 (2016).

37. D. Saxena et al., "Optically pumped room-temperature GaAs nanowire lasers," Nat. Photonics 7(12), 963-968 (2013).

38. G. T. Wang et al., "Highly aligned, template-free growth and characterization of vertical GaN nanowires on sapphire by metal-organic chemical vapour deposition," Nanotechnology 17(23), 5773-5780 (2006).

39. T. Kuykendall et al., "Metalorganic chemical vapor deposition route to $\mathrm{GaN}$ nanowires with triangular cross sections," Nano Lett. 3(8), 1063-1066 (2003).

40. A. K. Sivadasan et al., "Optical properties of monodispersed AlGaN nanowires in the single-prong growth mechanism," Cryst. Growth Des. 15(3), 1311-1318 (2015).

41. K. Hestroffer et al., "Polarity of GaN nanowires grown by plasma-assisted molecular beam epitaxy on Si(111)," Phys. Rev. B 84(24), 245302 (2011).

42. B. Janjua et al., "Self-planarized quantum-disks-in-nanowires ultraviolet-B emitters utilizing pendeo-epitaxy," Nanoscale 9(23), 7805-7813 (2017).

43. B. Janjua et al., "Ultraviolet-A LED based on quantum-disks-in-AlGaN-nanowires-optimization and device reliability," IEEE Photonics J. 10(2), 2200711 (2018).

44. K. H. Li et al., "Ultralow-threshold electrically injected AlGaN nanowire ultraviolet lasers on Si operating at low temperature," Nat. Nanotechnol. 10(2), 140-144 (2015).

45. M. Djavid and Z. Mi, "Enhancing the light extraction efficiency of AlGaN deep ultraviolet light emitting diodes by using nanowire structures," Appl. Phys. Lett. 108(5), 051102 (2016).

46. R. Lin et al., "Tapering-induced enhancement of light extraction efficiency of nanowire deep ultraviolet LED by theoretical simulations," Photonics Res. 6(5), 457-462 (2018).

47. S. M. Islam et al., "Deep-UV emission at $219 \mathrm{~nm}$ from ultrathin MBE GaN/AlN quantum heterostructures," Appl. Phys. Lett. 111(9), 091104 (2017).

48. J. Ristić et al., "GaN/AlGaN nanocavities with AlN/GaN Bragg reflectors grown in AlGaN nanocolumns by plasma assisted MBE," Phys. Status Solidi A 202(3), 367-371 (2005).

49. J. Verma et al., "Tunnel-injection GaN quantum dot ultraviolet light-emitting diodes," Appl. Phys. Lett. 102(4), 041103 (2013).

50. J. Verma et al., "Tunnel-injection quantum dot deep-ultraviolet light-emitting diodes with polarization-induced doping in III-nitride heterostructures," Appl. Phys. Lett. 104(2), 021105 (2014).

51. A. T. M. G. Sarwar et al., "Ultrathin GaN quantum disk nanowire LEDs with sub-250 nm electroluminescence," Nanoscale 8(15), 8024-8032 (2016).

52. S. M. Islam et al., "Sub-230 nm deep-UV emission from GaN quantum disks in AlN grown by a modified Stranski-Krastanov mode," Jpn. J. Appl. Phys. 55(5), 05FF06 (2016).

53. S. Tanaka, S. Iwai, and Y. Aoyagi, "Self-assembling GaN quantum dots on $\mathrm{Al}_{\mathrm{x}} \mathrm{Ga}_{1-\mathrm{x}} \mathrm{N}$ surfaces using a surfactant," Appl. Phys. Lett. 69(26), 4096-4098 (1996).

54. W. Seifert et al., "In-situ growth of quantum dot structures by the Stranski-Kranstanow growth mode," Prog. Cryst. Growth Ch. 33(4), 423-471 (1996).

55. N. Gogneau et al., "Structure of GaN quantum dots grown under 'modified StranskiKrastanow' conditions on AlN," J. Appl. Phys. 94(4), 2254-2261 (2003).

56. J. Ristić et al., "Characterization of $\mathrm{GaN}$ quantum discs embedded in $\mathrm{Al}_{\mathrm{x}} \mathrm{Ga}_{1-\mathrm{x}} \mathrm{N}$ nanocolumns grown by molecular beam epitaxy," Phys. Rev. B 68(12), 125305 (2003). 
57. J. Ristić et al., "Carrier-confinement effects in nanocolumnar $\mathrm{GaN} / \mathrm{Al}_{\mathrm{x}} \mathrm{Ga}_{1-\mathrm{x}} \mathrm{N}$ quantum disks grown by molecular-beam epitaxy," Phys. Rev. B 72(8), 085330 (2005).

58. S. Tanaka et al., "A UV light-emitting diode incorporating GaN quantum dots," Jpn. J. Appl. Phys. 42(8A), L885-L887 (2003).

59. Y. Taniyasu, M. Kasu, and T. Makimoto, "An aluminium nitride light-emitting diode with a wavelength of 210 nanometres," Nature 441(7091), 325-328 (2006).

60. H. Sekiguchi, K. Kishino, and A. Kikuchi, "GaN/AlGaN nanocolumn ultraviolet lightemitting diodes grown on n-(111) Si by RF-plasma-assisted molecular beam epitaxy," Electron. Lett. 44(2), 151-152 (2008).

61. L. Rigutti et al., "Ultraviolet photodetector based on GaN/AlN quantum disks in a single nanowire," Nano Lett. 10(8), 2939-2943 (2010).

62. D. Priante et al., "Highly uniform ultraviolet-A quantum-confined AlGaN nanowire LEDs on metal/silicon with a TaN interlayer," Opt. Mater. Express 7(12), 4214-4224 (2017).

63. M. Spies et al., "Bias-controlled spectral response in GaN/AlN single-nanowire ultraviolet photodetectors," Nano Lett. 17(7), 4231-4239 (2017).

64. J. Renard et al., "Suppression of nonradiative processes in long-lived polar GaN/AIN quantum dots," Appl. Phys. Lett. 95(13), 131903 (2009).

65. S. Zhao et al., "Sub-milliwatt AlGaN nanowire tunnel junction deep ultraviolet light emitting diodes on silicon operating at 242 nm," Appl. Phys. Lett. 109(20), 201106 (2016).

66. K. Balakrishnan et al., "Influence of high temperature in the growth of low dislocation content AlN bridge layers on patterned $6 \mathrm{H}-\mathrm{SiC}$ substrates by metalorganic vapor phase epitaxy," Jpn. J. Appl. Phys. 46(12-16), L307-L310 (2007).

67. Z. Chen et al., "Pulsed lateral epitaxial overgrowth of aluminum nitride on sapphire substrates," Appl. Phys. Lett. 89(8), 081905 (2006).

68. V. Adivarahan et al., "Robust $290 \mathrm{~nm}$ emission light emitting diodes over pulsed laterally overgrown AlN," Jpn. J. Appl. Phys. 46(36-40), L877-L879 (2007).

69. R. Jain et al., "Migration enhanced lateral epitaxial overgrowth of AlN and AlGaN for high reliability deep ultraviolet light emitting diodes," Appl. Phys. Lett. 93(5), 051113 (2008).

70. B. S. Cheng et al., "Light output enhancement of UV light-emitting diodes with embedded distributed Bragg reflector," IEEE Photonics Technol. Lett. 23(10), 642-644 (2011).

71. C.-Y. Huang et al., "MQWs InGaN/GaN LED with embedded micro-mirror array in the epitaxial-lateral-overgrowth gallium nitride for light extraction enhancement," Opt. Express 18(10), 10674-10684 (2010).

72. M. Tangi, A. De, and S. M. Shivaprasad, "Role of dislocations and carrier concentration in limiting the electron mobility of InN films grown by plasma assisted molecular beam epitaxy," J. Appl. Phys. 123(1), 015701 (2018).

73. M. Conroy et al., "Epitaxial lateral overgrowth of AlN on self-assembled patterned nanorods," J. Mater. Chem. C 3(2), 431-437 (2015).

74. D. Lee et al., "Improved performance of AlGaN-based deep ultraviolet light-emitting diodes with nano-patterned AlN/sapphire substrates," Appl. Phys. Lett. 110(19), 191103 (2017).

75. V. Kueller et al., "Modulated epitaxial lateral overgrowth of AIN for efficient UV LEDs," IEEE Photonics Technol. Lett. 24(18), 1603-1605 (2012).

76. H. Yoshida et al., "A 342-nm ultraviolet AlGaN multiple-quantum-well laser diode," Nat. Photonics 2(9), 551-554 (2008).

77. A. Usui et al., "Thick GaN epitaxial growth with low dislocation density by hydride vapor phase epitaxy," Jpn. J. Appl. Phys. 36(7B), L899-L902 (1997).

78. P. Gupta et al., "Layered transition metal dichalcogenides: promising near-lattice-matched substrates for GaN growth," Sci. Rep. 6(1), 23708 (2016).

79. M. Tangi et al., "Determination of band offsets at GaN/single-layer $\mathrm{MoS}_{2}$ heterojunction," Appl. Phys. Lett. 109(3), 032104 (2016).

80. P. Mishra et al., "Impact of N-plasma and Ga-irradiation on $\mathrm{MoS}_{2}$ layer in molecular beam epitaxy," Appl. Phys. Lett. 110(1), 012101 (2017).

81. M. Tangi et al., "Band alignment at GaN/single-layer $\mathrm{WSe}_{2}$ interface," ACS Appl. Mater. Interfaces 9(10), 9110-9117 (2017). 
82. Y. Sun et al., "Nucleation mechanism of GaN growth on wet etched pattern sapphire substrates," CrystEngComm 16(24), 5458-5465 (2014).

83. J. B. Kim et al., "Light extraction enhancement of GaN-based light-emitting diodes using volcano-shaped patterned sapphire substrates," Jpn. J. Appl. Phys. 49(4R), 042102 (2010).

84. Z. Liu et al., "Fabrication of $\mathrm{GaN}$ hexagonal cones by inductively coupled plasma reactive ion etching," J. Vac. Sci. Technol. B 34(4), 041226 (2016).

85. C. C. Pan et al., "Light output improvement of InGaN ultraviolet light-emitting diodes by using wet-etched stripe-patterned sapphire substrates," J. Appl. Phys. 102(8), 084503 (2007).

86. P. Dong et al., " $282-\mathrm{nm}$ AlGaN-based deep ultraviolet light-emitting diodes with improved performance on nano-patterned sapphire substrates," Appl. Phys. Lett. 102(24), 241113 (2013).

87. M. Khizar et al., "Nitride deep-ultraviolet light-emitting diodes with microlens array," Appl. Phys. Lett. 86(17), 173504 (2005).

88. M. Kim et al., "AlGaN-based deep ultraviolet light-emitting diodes fabricated on patterned sapphire substrates," Appl. Phys. Express 4(9), 092102 (2011).

89. X. Chen et al., "Deep-ultraviolet stimulated emission from AlGaN/AlN multiple-quantumwells on nano-patterned AlN/sapphire templates with reduced threshold power density," J. Alloys Compd. 723, 192-196 (2017).

90. S. I. Inoue, N. Tamari, and M. Taniguchi, " $150 \mathrm{~mW}$ deep-ultraviolet light-emitting diodes with large-area AlN nanophotonic light-extraction structure emitting at $265 \mathrm{~nm}, " \mathrm{Appl}$. Phys. Lett. 110(14), 141106 (2017).

91. S. I. Inoue et al., "Light extraction enhancement of $265 \mathrm{~nm}$ deep-ultraviolet light-emitting diodes with over $90 \mathrm{~mW}$ output power via an AlN hybrid nanostructure," Appl. Phys. Lett. 106(13), 131104 (2015).

92. J. Kim et al., "Deep-ultraviolet AlGaN/AlN core-shell multiple quantum wells on AlN nanorods via lithography-free method," Sci. Rep. 8(1), 935 (2018).

93. Y. J. Lee et al., "Improvement in light-output efficiency of near-ultraviolet InGaN-GaN LEDs fabricated on stripe patterned sapphire substrate," in Pacific Rim Conf. on Lasers and Electro-Optics (CLEO-PR), CWAB3-P53 (2005).

94. S. Zhou et al., "Comparative study of GaN-based ultraviolet LEDs grown on differentsized patterned sapphire substrates with sputtered AlN nucleation layer," Jpn. J. Appl. Phys. 56(11), 111001 (2017).

95. D. Alden et al., "Fabrication and structural properties of AlN submicron periodic lateral polar structures and waveguides for UV-C applications," Appl. Phys. Lett. 108(26), 261106 (2016).

96. H. J. Kang et al., "Improving light-emitting diode performance through sapphire substrate double-side patterning," Opt. Eng. 52(2), 023002 (2013).

97. X.-F. Li et al., "Fabrication of patterned sapphire substrate and effect of light emission pattern on package efficiency," Opt. Mater. Express 5(8), 1784-1791 (2015).

98. Y. Guo et al., "Light extraction enhancement of AlGaN-based ultraviolet light-emitting diodes by substrate sidewall roughening," Appl. Phys. Lett. 111(1), 011102 (2017).

99. Y. Zhang et al., "Near milliwatt power AlGaN-based ultraviolet light emitting diodes based on lateral epitaxial overgrowth of AlN on Si(111)," Appl. Phys. Lett. 102(1), 011106 (2013).

100. B. T. Tran and H. Hirayama, "Growth and fabrication of high external quantum efficiency AlGaN-based deep ultraviolet light-emitting diode grown on pattern Si substrate," Sci. Rep. 7(1), 12176 (2017).

101. İ. Demir et al., "Direct growth of thick AlN layers on nanopatterned Si substrates by cantilever epitaxy," Phys. Status Solidi A 214(4), 1600363 (2017).

102. C. E. Lee et al., "Output power enhancement of vertical-injection ultraviolet light-emitting diodes by GaN-free and surface roughness structures," Electrochem. Solid-State Lett. 12(2), H44-H46 (2009).

103. Y. C. Chiang et al., "Enhanced performance of nitride-based ultraviolet vertical-injection light-emitting diodes by non-insulation current blocking layer and textured surface," Nanoscale Res. Lett. 9(1), 699 (2014). 
104. Y. C. Chiang, C. C. Lin, and H. C. Kuo, "Novel thin-GaN LED structure adopted micro abraded surface to compare with conventional vertical LEDs in ultraviolet light," Nanoscale Res. Lett. 10(1), 182 (2015).

105. L. Zhou et al., "Vertical injection thin-film AlGaN/AlGaN multiple-quantum-well deep ultraviolet light-emitting diodes," Appl. Phys. Lett. 89(24), 241113 (2006).

106. B. J. Kim et al., "Enhancement of light extraction efficiency of ultraviolet light emitting diodes by patterning of $\mathrm{SiO}_{2}$ nanosphere arrays," Thin Solid Films 517(8), 2742-2744 (2009).

107. X. Liu et al., "Molecular beam epitaxial growth and characterization of AIN nanowall deep UV light emitting diodes," Appl. Phys. Lett. 111(10), 101103 (2017).

108. W. Guo et al., "Enhancing light coupling and emission efficiencies of AlGaN thin film and $\mathrm{AlGaN} / \mathrm{GaN}$ multiple quantum wells with periodicity-wavelength matched nanostructure array," Nanoscale 9(40), 15477-15483 (2017).

109. Y. Peng et al., "Light efficiency enhancement of deep ultraviolet light-emitting diodes packaged by nanostructured silica glass," J. Disp. Technol. 12(10), 1106-1111 (2016).

110. J. W. Lee et al., "Arrays of truncated cone AlGaN deep-ultraviolet light-emitting diodes facilitating efficient outcoupling of in-plane emission," ACS Photonics 3(11), 2030-2034 (2016).

111. T. Takano et al., "Deep-ultraviolet light-emitting diodes with external quantum efficiency higher than $20 \%$ at $275 \mathrm{~nm}$ achieved by improving light-extraction efficiency," Appl. Phys. Express 10(3), 031002 (2017).

112. G. B. Fayisa et al., "Enhanced light extraction efficiency of micro-ring array AlGaN deep ultraviolet light-emitting diodes," Jpn. J. Appl. Phys. 56(9), 092101 (2017).

113. Y. Peng et al., "Enhanced light extraction from DUV-LEDs by AlN-doped fluoropolymer encapsulation," IEEE Photonics Technol. Lett. 29(14), 1151-1154 (2017).

114. S. Nagai et al., "Development of highly durable deep-ultraviolet AlGaN-based LED multichip array with hemispherical encapsulated structures using a selected resin through a detailed feasibility study," Jpn. J. Appl. Phys. 55(8), 082101 (2016).

115. S. Wu et al., "Micro-pixel design milliwatt power $254 \mathrm{~nm}$ emission light emitting diodes," Jpn. J. Appl. Phys. 43(8A), L1035-L1037 (2004).

116. N. Lobo et al., "Enhancement of light extraction in ultraviolet light-emitting diodes using nanopixel contact design with Al reflector," Appl. Phys. Lett. 96(8), 081109 (2010).

117. S. M. Mitani, P. K. Choudhury, and M. S. Alias, "On the characterization of a new type of oxide-confined $850 \mathrm{~nm}$ GaAs-based vertical-cavity surface-emitting laser," Optik 119(8), 373-378 (2008).

118. M. S. Alias et al., "Improved efficiency of GaAs-based VCSEL by utilizing fan-pad metallization and trench patterning," Laser Phys. 19(11), 2096-2101 (2009).

119. M. S. Alias, S. Shaari, and S. M. Mitani, "Optimization of electro-optical characteristics of GaAs-based oxide confinement VCSEL," Laser Phys. 20(4), 806-810 (2010).

120. A. N. Al-Omari et al., "Improved performance of top-emitting oxide-confined polyimideplanarized $980 \mathrm{~nm}$ VCSELs with copper-plated heat sinks," J. Phys. D Appl. Phys. 45(50), 505101 (2012).

121. D. Priante et al., "The recombination mechanisms leading to amplified spontaneous emission at the true-green wavelength in $\mathrm{CH}_{3} \mathrm{NH}_{3} \mathrm{PbBr}_{3}$ perovskites," Appl. Phys. Lett. 106(8), 081902 (2015).

122. M. S. Alias et al., "Continuous-wave optically pumped green perovskite vertical-cavity surface-emitter," Opt. Lett. 42(18), 3618-3621 (2017).

123. B. Murali et al., "Temperature-induced lattice relaxation of perovskite crystal enhances optoelectronic properties and solar cell performance," J. Phys. Chem. Lett. 8(1), 137143 (2017).

124. M. S. Alias and S. Shaari, "Analysis of single-mode condition and high-order modes discrimination in photonic crystal VCSEL," Appl. Phys. B 103(4), 889-896 (2011).

125. M. S. Alias et al., "Single transverse mode control of VCSEL by photonic crystal and trench patterning," Photonics Nanostruct. 8(1), 38-46 (2010).

126. M. S. Alias et al., "Highly confined and continuous single-mode operation of self-align photonic crystal oxide VCSEL," Appl. Phys. B 100(3), 453-459 (2010). 
127. M. S. Alias and S. Shaari, "Loss analysis of high order modes in photonic crystal verticalcavity surface-emitting lasers," J. Light. Technol. 28(10), 1556-1563 (2010).

128. P. O. Leisher et al., "High modulation bandwidth implant-confined photonic crystal vertical-cavity surface-emitting lasers," IEEE Photonics Technol. Lett. 19(19), 1541-1543 (2007).

129. J. Y. Zhang et al., "Blue-violet lasing of optically pumped GaN-based vertical cavity surface-emitting laser with dielectric distributed Bragg reflectors," J. Light. Technol. 27(1), 55-59 (2009).

130. T. Someya et al., "Lasing emission from an $\mathrm{In}_{0.1} \mathrm{Ga}_{0.9} \mathrm{~N}$ vertical cavity surface emitting laser," Jpn. J. Appl. Phys. 37(12A), L1424-L1426 (1998).

131. Y. S. Liu et al., "Optically pumped vertical-cavity surface-emitting laser at $374.5 \mathrm{~nm}$ with an electrically conducting n-DBR," Appl. Phys. Express 9(11), 111002 (2016).

132. T. Moudakir et al., "Design, fabrication, and characterization of near-milliwatt-power RCLEDs emitting at 390 nm," IEEE Photonics J. 5(6), 8400709 (2013).

133. Z. Xie et al., "AlGaN-based $330 \mathrm{~nm}$ resonant-cavity-enhanced $\mathrm{p}-\mathrm{i}-\mathrm{n}$ junction ultraviolet photodetectors using AlN/AlGaN distributed Bragg reflectors," Phys. Status Solidi C 7(78), 1821-1824 (2010).

134. Y. Ma et al., "Enhanced light output of near-ultraviolet LEDs with $\mathrm{Ta}_{2} \mathrm{O}_{5} / \mathrm{SiO}_{2}$ hybrid DBR reflector," IEEE Photonics Technol. Lett. 29(18), 1564-1567 (2017).

135. G. J. Wang et al., "GaN/AlGaN ultraviolet light-emitting diode with an embedded porousAlGaN distributed Bragg reflector," Appl. Phys. Express 10(12), 122102 (2017).

136. T. Wang et al., "Highly improved performance of a $350 \mathrm{~nm}$ ultraviolet light-emitting diode containing $\mathrm{Al}_{\mathrm{x}} \mathrm{Ga}_{1-\mathrm{x}} \mathrm{N} / \mathrm{Al}_{\mathrm{y}} \mathrm{Ga}_{1-\mathrm{y}} \mathrm{N}$ distributed Bragg reflectors," J. Cryst. Growth 267(34), 583-587 (2004).

137. C. C. Chen et al., "Ultraviolet GaN-based microdisk laser with AlN/AlGaN distributed Bragg reflector," Appl. Phys. Lett. 96(15), 151115 (2010).

138. M. Liu et al., "Comparative experimental and simulation studies of high-power AlGaNbased $353 \mathrm{~nm}$ ultraviolet flip-chip and top-emitting LEDs," Jpn. J. Appl. Phys. 57(3), 031001 (2018).

139. T. T. Kao et al., "Sub- $250 \mathrm{~nm}$ low-threshold deep-ultraviolet AlGaN-based heterostructure laser employing $\mathrm{HfO}_{2} / \mathrm{SiO}_{2}$ dielectric mirrors," Appl. Phys. Lett. 103(21), 211103 (2013).

140. T. Someya et al., "Room temperature lasing at blue wavelengths in gallium nitride microcavities," Science 285(5435), 1905-1906 (1999).

141. A. Alyamani et al., "GaN hybrid microcavities in the strong coupling regime grown by metal-organic chemical vapor deposition on sapphire substrates," J. Appl. Phys. 101(9), 093110 (2007).

142. M. L. Grilli et al., " $\mathrm{Al}_{2} \mathrm{O}_{3} / \mathrm{SiO}_{2}$ and $\mathrm{HfO}_{2} / \mathrm{SiO}_{2}$ dichroic mirrors for UV solid-state lasers," Thin Solid Films 517(5), 1731-1735 (2009).

143. P. Torchio et al., "High-reflectivity $\mathrm{HfO}_{2} / \mathrm{SiO}_{2}$ ultraviolet mirrors," Appl. Opt. 41(16), 3256-3261 (2002).

144. M. S. Alias et al., "High reflectivity $\mathrm{YDH} / \mathrm{SiO}_{2}$ distributed Bragg reflector for UV-C wavelength regime," IEEE Photonics J. 10(2), 2200508 (2018).

145. M. S. Alias et al., "Optical constants of $\mathrm{CH}_{3} \mathrm{NH}_{3} \mathrm{PbBr}_{3}$ perovskite thin films measured by spectroscopic ellipsometry," Opt. Express 24(15), 16586-16594 (2016).

146. G. S. Huang et al., "Crack-free GaN/AlN distributed Bragg reflectors incorporated with GaN/AlN superlattices grown by metalorganic chemical vapor deposition," Appl. Phys. Lett. 88(6), 061904 (2006).

147. D. Wang et al., "Crack-free ultraviolet AlGaN/GaN distributed Bragg reflectors grown by MOVPE on 6H-SiC(0 00 1)," Superlattices Microstruct. 70, 54-60 (2014).

148. T. Someya and Y. Arakawa, "Highly reflective $\mathrm{GaN} / \mathrm{Al}_{0.34} \mathrm{Ga}_{0.6} \mathrm{~N}$ quarter-wave reflectors grown by metal organic chemical vapor deposition," Appl. Phys. Lett. 73(25), 3653-3655 (1998).

149. T. Moudakir et al., "Suppression of crack generation in $\mathrm{AlGaN} / \mathrm{GaN}$ distributed Bragg reflectors grown by MOVPE," J. Cryst. Growth 370, 12-15 (2013).

150. K. E. Waldrip et al., "Stress engineering during metalorganic chemical vapor deposition of AlGaN/GaN distributed Bragg reflectors," Appl. Phys. Lett. 78(21), 3205-3207 (2001). 
151. Y. S. Liu et al., "Strain management of AlGaN-based distributed Bragg reflectors with GaN interlayer grown by metalorganic chemical vapor deposition," Appl. Phys. Lett. 109(8), 081103 (2016).

152. A. Bhattacharyya et al., "High reflectivity and crack-free AlGaN/AlN ultraviolet distributed Bragg reflectors," J. Vac. Sci. Technol. B 20(3), 1229-1233 (2002).

153. A. Franke et al., "High reflectivity III-nitride UV-C distributed Bragg reflectors for vertical cavity emitting lasers," J. Appl. Phys. 120(13), 135703 (2016).

154. A. Franke et al., "Strain engineered high reflectivity DBRs in the deep UV," Proc. SPIE 9748, 97481G (2016).

155. M. Abid et al., "Distributed Bragg reflectors based on diluted boron-based BAIN alloys for deep ultraviolet optoelectronic applications," Appl. Phys. Lett. 100(5), 051101 (2012).

156. C. Berger et al., "In-situ growth monitoring of AlInN/AlGaN distributed Bragg reflectors for the UV-spectral range," J. Cryst. Growth 370, 87-91 (2013).

157. C. Berger et al., "Growth of AlInN/AlGaN distributed Bragg reflectors for high quality microcavities," Phys. Status Solidi C 9(5), 1253-1258 (2012).

158. E. Feltin et al., "Crack-free highly reflective AlInN/AlGaN Bragg mirrors for UV applications," Appl. Phys. Lett. 88(5), 051108 (2006).

159. L. Zhang et al., "Solar-blind ultraviolet AlInN/AlGaN distributed Bragg reflectors," Appl. Phys. Lett. 102(24), 242112 (2013).

160. J. Chang et al., "High-quality crystal growth and characteristics of AlGaN-based solarblind distributed Bragg reflectors with a tri-layer period structure," Sci. Rep. 6(1), 29571 (2016).

161. Y. Kashima et al., "High external quantum efficiency (10\%) AlGaN-based deep-ultraviolet light-emitting diodes achieved by using highly reflective photonic crystal on p-AlGaN contact layer," Appl. Phys. Express 11(1), 012101 (2018).

162. J. B. Shakya, "III-nitride blue and UV photonic-crystal light-emitting diodes," Proc. SPIE 5530, 241-250 (2004).

163. T. N. Oder et al., "III-nitride blue and ultraviolet photonic crystal light emitting diodes," Appl. Phys. Lett. 84(4), 466-468 (2004).

164. J. Shakya, J. Y. Lin, and H. X. Jiang, "Time-resolved electroluminescence studies of III-nitride ultraviolet photonic-crystal light-emitting diodes," Appl. Phys. Lett. 85(11), 2104-2106 (2004).

165. D. Néel et al., "Aluminum nitride photonic crystals and microdiscs for ultra-violet nanophotonics," Adv. Nat. Sci. Nanosci. 5(2), 023001 (2014).

166. C. C. Chen et al., "Tunable light emission from GaN-based photonic crystal with ultraviolet AlN/AlGaN distributed Bragg reflector," Jpn. J. Appl. Phys. 50(45), 04DG09 (2011).

167. C. C. Chen et al., "Light emission enhancement of GaN-based photonic crystal with ultraviolet AlN/AlGaN distributed Bragg reflector," J. Lightwave Technol. 28(22), 3189-3192 (2010).

168. J. Zhang et al., "Fabrication and photovoltaic measurements of surface nanostructure of AlGaInN-based photodetector," Jpn. J. Appl. Phys. 48(11R), 111001 (2009).

169. X.-L. Hu et al., "III-nitride ultraviolet, blue and green $\mathrm{LEDs}$ with $\mathrm{SiO}_{2}$ photonic crystals fabricated by UV-nanoimprint lithography," Mater. Sci. Semicond. Process. 79, 61-65 (2018).

170. J. Shakya et al., "Enhanced light extraction in III-nitride ultraviolet photonic crystal lightemitting diodes," Appl. Phys. Lett. 85(1), 142-144 (2004).

171. Z. Y. Fan, J. Y. Lin, and H. X. Jiang, "III-nitride deep ultraviolet micro- and nano-photonics," Proc. SPIE 6127, 61271C (2006).

172. M. S. Alias et al., "Enhancing the light-extraction efficiency of an AlGaN nanowire ultraviolet light-emitting diode by using nitride/air distributed Bragg reflector nanogratings," IEEE Photonics J. 9(5), 4900508 (2017).

173. M. S. Alias et al., "Enhanced etching, surface damage recovery, and submicron patterning of hybrid perovskites using a chemically gas-assisted focused-ion beam for subwavelength grating photonic applications," J. Phys. Chem. Lett. 7(1), 137-142 (2016). 
174. M. S. Alias et al., "Focused-ion beam patterning of organolead trihalide perovskite for subwavelength grating nanophotonic applications," J. Vac. Sci. Technol. B 33(5), 051207 (2015).

175. Y. Takashima et al., "Ultraviolet polarizer with a Ge subwavelength grating," Appl. Opt. 56(29), 8224-8229 (2017).

176. T. Weber et al., "High aspect ratio deep UV wire grid polarizer fabricated by double patterning," Microelectron. Eng. 98, 433-435 (2012).

177. T. Weber et al., "Iridium wire grid polarizer fabricated using atomic layer deposition," Nanoscale Res. Lett. 6, 558 (2011).

178. T. Siefke et al., "Materials pushing the application limits of wire grid polarizers further into the deep ultraviolet spectral range," Adv. Opt. Mater. 4(11), 1780-1786 (2016).

179. T. Weber et al., "Tungsten wire grid polarizer for applications in the DUV spectral range," Appl. Opt. 51(16), 3224-3227 (2012).

180. T. Weber et al., "Silicon wire grid polarizer for ultraviolet applications," Appl. Opt. 53(34), 8140-8144 (2014).

181. K. Asano et al., "Fabrication and characterization of a deep ultraviolet wire grid polarizer with a chromium-oxide subwavelength grating," Appl. Opt. 53(13), 2942-2948 (2014).

182. Y. Takashima et al., "Highly polarized emission from a GaN-based ultraviolet lightemitting diode using a $\mathrm{Si}$-subwavelength grating on a $\mathrm{SiO}_{2}$ underlayer," Opt. Commun. 369, 38-43 (2016).

183. Y. Takashima et al., "Polarized emission characteristics of UV-LED with subwavelength grating," Jpn. J. Appl. Phys. 53(7), 072101 (2014).

184. G. Miyaji and K. Miyazaki, "Fabrication of 50-nm period gratings on $\mathrm{GaN}$ in air through plasmonic near-field ablation induced by ultraviolet femtosecond laser pulses," Opt. Express 24(5), 4648-4653 (2016).

185. K. J. Chen et al., "Lasing characteristics of a metal-coated GaN shallow grating structure at room temperature," IEEE J. Sel. Top. Quantum Electron. 21(1), 4600105 (2015).

186. T. S. Kim et al., "Increase of light extraction from $\mathrm{GaN}$ based light emitting diodes incorporating patterned structure by colloidal lithography," Appl. Phys. Lett. 91(17), 171114 (2007).

187. D.-S. Leem et al., "Light-output enhancement of GaN-based light-emitting diodes by using hole-patterned transparent indium tin oxide electrodes," J. Appl. Phys. 98(7), 076107 (2005).

188. S. Butun, N. A. Cinel, and E. Ozbay, "Nanoantenna coupled UV subwavelength photodetectors based on GaN," Opt. Express 20(3), 2649-2656 (2012).

189. H. G. Hong et al., "Enhancement of the light output of GaN-based ultraviolet light-emitting diodes by a one-dimensional nanopatterning process," Appl. Phys. Lett. 88(10), 103505 (2006).

190. H.-G. Hong et al., "Enhanced light output of GaN-based near-UV light-emitting diodes by using nanopatterned indium tin oxide electrodes," Semicond. Sci. Technol. 21(5), 594-597 (2006).

191. Z. Zhuang et al., "Large-scale fabrication and luminescence properties of $\mathrm{GaN}$ nanostructures by a soft UV-curing nanoimprint lithography," Nanotechnology 24(40), 405303 (2013).

192. K.-J. Byeon and H. Lee, "Recent progress in direct patterning technologies based on nanoimprint lithography," Eur. Phys. J. Appl. Phys. 59(1), 10001 (2012).

193. H. X. Jiang and J. Y. Lin, "Advances in III-nitride microstructures and micro-size emitters," J. Korean Phys. Soc. 42(9), S757-S764 (2003).

194. Y. Zhang et al., "Advances in III-nitride semiconductor microdisk lasers," Phys. Status Solidi A 212(5), 960-973 (2015).

195. R. A. Mair et al., "Optical properties of GaN/AlGaN multiple quantum well microdisks," Appl. Phys. Lett. 71(20), 2898-2900 (1997).

196. M. Kneissl et al., "Current-injection spiral-shaped microcavity disk laser diodes with unidirectional emission," Appl. Phys. Lett. 84(14), 2485-2487 (2004).

197. H. W. Choi et al., "Lasing in GaN microdisks pivoted on Si," Appl. Phys. Lett. 89(21), 211101 (2006). 
198. G. Zhu et al., "Single-mode ultraviolet whispering gallery mode lasing from a floating GaN microdisk," Opt. Lett. 43(4), 647-650 (2018).

199. Y. Zhang et al., "Optically-pumped single-mode deep-ultraviolet microdisk lasers with AlGaN-based multiple quantum wells on Si substrate," IEEE Photonics J. 9(5), 2400508 (2017).

200. J. Selles et al., "Deep-UV nitride-on-silicon microdisk lasers," Sci. Rep. 6(1), 21650 (2016).

201. R. A. Mair et al., "Optical modes within III-nitride multiple quantum well microdisk cavities," Appl. Phys. Lett. 72(13), 1530-1532 (1998).

202. S. Chang et al., "Stimulated emission and lasing in whispering-gallery modes of GaN microdisk cavities," Appl. Phys. Lett. 75(2), 166-168 (1999).

203. S. Chang et al., "GaN near-UV microdisk lasers: efficient pumping configuration and low lasing threshold," in Conf. on Lasers and Electro-Optics (CLEO), CTuU6 (1999).

204. N. B. Rex et al., "Directional stimulated emission from deformed GaN micropillar cavities," in 12th Annual Meeting IEEE Lasers and Electro-Optics Society (LEOS), WT2 (1999).

205. N. B. Rex et al., "Directional laser emission from chaotic modes in quadrupole-deformed GaN microdisks," in Conf. on Lasers and Electro-Optics (CLEO), CTuA57 (2000).

206. S. Sergent et al., "GaN quantum dots in (Al, Ga)N-based microdisks," J. Phys. Conf. Ser. 210(1), 012005 (2010).

207. M. Mexis et al., "High quality factor nitride-based optical cavities: microdisks with embedded GaN/Al(Ga)N quantum dots," Opt. Lett. 36(12), 2203-2205 (2011).

208. M. Bürger et al., "Cubic GaN quantum dots embedded in zinc-blende AlN microdisks," J. Cryst. Growth 378, 287-290 (2013).

209. M. Bürger et al., "Whispering gallery modes in zinc-blende AlN microdisks containing non-polar GaN quantum dots," Appl. Phys. Lett. 102(8), 081105 (2013).

210. M. Bürger et al., "Lasing properties of non-polar GaN quantum dots in cubic aluminum nitride microdisk cavities," Appl. Phys. Lett. 103(2), 021107 (2013).

211. J. Sellés et al., "III-nitride-on-silicon microdisk lasers from the blue to the deep ultra-violet," Appl. Phys. Lett. 109(23), 231101 (2016).

212. Y. Zhang et al., "UV-C whispering-gallery modes in AlN microdisks with AlGaN-based multiple quantum wells on Si substrate," J. Nanophotonics 12(4), 043502 (2018).

213. R. Chen et al., "Optically pumped ultraviolet lasing from nitride nanopillars at room temperature," Appl. Phys. Lett. 96(24), 241101 (2010).

214. K. H. Li, Z. Ma, and H. W. Choi, "Single-mode whispering gallery lasing from metal-clad GaN nanopillars," Opt. Lett. 37(3), 374-376 (2012).

215. Q. Li et al., "Single-mode GaN nanowire lasers," Opt. Express 20(16), 17873-17879 (2012).

216. H. Xu et al., "Single-mode lasing of GaN nanowire-pairs," Appl. Phys. Lett. 101(11), 113106 (2012).

217. H. Xu et al., "Gold substrate-induced single-mode lasing of GaN nanowires," Appl. Phys. Lett. 101(22), 221114 (2012).

218. Y. C. Hsu et al., "Room temperature ultraviolet GaN metal-coated nanorod laser," Appl. Phys. Lett. 103(19), 191102 (2013).

219. J. B. Wright et al., "Distributed feedback gallium nitride nanowire lasers," Appl. Phys. Lett. 104(4), 041107 (2014).

220. G. Griffel, "Vernier effect in asymmetrical ring resonator arrays," IEEE Photonics Technol. Lett. 12(12), 1642-1644 (2000).

221. K. C. Zeng et al., "Optical resonance modes in InGaN/GaN multiple-quantum-well microring cavities," Appl. Phys. Lett. 75(17), 2563-2565 (1999).

222. Y. G. Wang et al., "Room temperature lasing with high group index in metal-coated GaN nanoring," Appl. Phys. Lett. 99(25), 251111 (2011).

223. Y. G. Wang et al., "Lasing in metal-coated GaN nanostripe at room temperature," Appl. Phys. Lett. 98(13), 131110 (2011).

224. Y. Zhang et al., "GaN hemispherical micro-cavities," Appl. Phys. Lett. 108(3), 031110 (2016). 
225. L. C. Wang et al., "Optically pumped lasing with a Q-factor exceeding 6000 from wetetched GaN micro-pyramids," Opt. Lett. 42(15), 2976-2979 (2017).

226. W. C. Liao et al., "Optimized spiral metal-gallium-nitride nanowire cavity for ultrahigh circular dichroism ultraviolet lasing at room temperature," Sci. Rep. 6(1), 26578 (2016).

227. M. Honda et al., "UV plasmonic device for sensing ethanol and acetone," Appl. Phys. Express 11(1), 012001 (2018).

228. K. Appusamy, "Development of materials for ultraviolet plasmonics," ProQuest Dissertations and Theses Global, 1839275333, University of Utah, Ann Arbor (2016).

229. P. Törmö and W. L. Barnes, "Strong coupling between surface plasmon polaritons and emitters: a review," Rep. Prog. Phys. 78(1), 013901 (2015).

230. N. Gao et al., "Surface-plasmon-enhanced deep-UV light emitting diodes based on AlGaN multi-quantum wells," Sci. Rep. 2(1), 816 (2012).

231. J. Lin et al., "Surface plasmon enhanced UV emission in AlGaN/GaN quantum well," Appl. Phys. Lett. 97(22), 221104 (2010).

232. C.-Y. Cho et al., "Surface plasmon enhanced light emission from AlGaN-based ultraviolet light-emitting diodes grown on Si(111)," Appl. Phys. Lett. 102(21), 211110 (2013).

233. K. C. Shen et al., "Giant enhancement of emission efficiency and light directivity by using hyperbolic metacavity on deep-ultraviolet AlGaN emitter," Nano Energy 45, 353-358 (2018).

234. K. R. Son et al., "Enhanced light emission from $\mathrm{AlGaN} / \mathrm{GaN}$ multiple quantum wells using the localized surface plasmon effect by aluminum nanoring patterns," Photonics Res. 6(1), 30-36 (2018).

235. K. Huang et al., "Top- and bottom-emission-enhanced electroluminescence of deep-UV light-emitting diodes induced by localised surface plasmons," Sci. Rep. 4(1), 4380 (2014).

236. A. Shetty et al., "Plasmonic enhancement of photocurrent in GaN based UV photodetectors," in IEEE 2nd Int. Conf. on Emerging Electronics: Materials to Devices (ICEE) (2014).

Mohd Sharizal Bin Alias is a research scientist at Photonics Laboratory, KAUST. He received his $\mathrm{PhD}$ (Microengineering and Nanoelectronics) degree from the National University of Malaysia in 2010, and his MSc (Physics) and BSc (Physics) degrees from the University of Malaya in 2005 and 2000, respectively. His research focuses on the usage of nanophotonics to enhance light manipulation for photonic devices. His other research interests include semiconductor lasers and micro-/nano-fabrication process. He is a senior member of IEEE.

Malleswararao Tangi is a postdoctoral research fellow at Photonics Laboratory, KAUST. He received his $\mathrm{PhD}$ in physics from Jawaharlal Nehru Center for advanced scientific research (JNCASR), India and masters in physics from University of Hyderabad, India. His work involves the molecular beam epitaxy growth of III-N materials, 2-D/3-D heterojunctions, and optoelectronic devices.

Jorge A. Holguin-Lerma received his college degree in electromedical engineering from Universidad La Salle Chihuahua, Mexico (2013), and his MS degree in materials science and engineering from King Abdullah University of Science and Technology (KAUST), Saudi Arabia (2016). Currently, he is PhD student in Photonics and Optoelectronics (EE) at KAUST. He is interested in the fields of nanophotonics and optoelectronic devices. He has participated in the industry in the area of device characterization as a components engineer at Honeywell ACS.

Edgars Stegenburgs is a PhD student at Photonics Laboratory, KAUST. He received his MSc degree (electrical engineering) from KAUST in 2016 and his BSc (physics) degree from the University of Latvia in 2014. Previously, he was working in the Laser Centre of the University of Latvia. His research focuses on manipulation of light in photonics and optoelectronics. Other research interests include light-matter interaction, numerical analysis, and solidstate lighting applications. He is a member of IEEE, OSA, and SPIE. 
Abdullah A. Alatawi is a PhD student at Photonics Laboratory, KAUST. He is working on different lab projects related to semiconductor devices fabrication and characterization. He received his master of science in optical nanotechnology engineering from Kassel University in Germany. He received his BSc degree in aerospace engineering from King Fahad University of Petroleum and Minerals and then he joined space institute at King Abdullaziz City for Science and Technology. He is a member of IEEE.

Islam Ashry is a postdoctoral research fellow at Photonics Laboratory, KAUST. He received his BS and MS degrees from the University of Alexandria, Egypt, in 2003 and 2007, respectively, and the PhD degree from Virginia Tech, VA, USA, in December 2012. His research interests include optical sensors, fluorescence dynamics, nanopatterning, plasmon sensing, nonlinear optics, fiber optics, optical communications, optical networks, and polymeric thin films. He is a member of IEEE.

Ram Chandra Subedi is a second-year PhD student in Photonics Laboratory at KAUST in CEMSE division. He received his MS (physics) degree from University of Georgia, USA in 2016. Also, he holds MSc (physics) and BSc (physics with Statistics minor) degrees from Tribhuvan University, Nepal in 2011 and 2007, respectively. Deep UV optoelectronic devices based on III-nitride is the main focus of his research.

Davide Priante received his BS degree in material science and engineering from Padua University, Italy, (2013) and his master's degree in electrical engineering from King Abdullah University of Science and Technology (KAUST) (2015). Currently, he is pursuing his $\mathrm{PhD}$ in Photonics Lab under the supervision of Prof. Ooi with a research focus on III-nitride wide-bandgap optoelectronic semiconductor devices. He is a member of OSA and SPIE.

Mohammad Khaled Shakfa is currently a postdoctoral research fellow in the Photonics Lab. at KAUST. Before joining KAUST, from 2015 to 2016, he conducted postdoctoral research in the workgroup of Experimental Semiconductor Physics at the Phillips Univ. of Marburg. In 2015, he received his $\mathrm{PhD}$ in optoelectronics from the Technical Univ. of Braunschweig. He earned his BS degree in physical sciences (2005) and his postgraduate diploma degree in Solid-State Physics (2006) from Al-Baath Univ. The research activities of Dr. Shakfa have focused on optics and optical spectroscopy.

Tien Khee Ng (senior member of IEEE, member of SPIE, and senior member of OSA) received his PhD (2005) and MEng (2001) degrees in electrical and electronic engineering from Nanyang Technological University, Singapore. Currently, he is a senior research scientist with Ooi-group at KAUST, Saudi Arabia, and the co-principal-investigator responsible for Molecular Beam Epitaxy thrust for the KACST Technology Innovation Center for Solid-State Lighting at KAUST.

Boon S. Ooi is a professor of electrical engineering at KAUST. He received his PhD in electronics and electrical engineering from the University of Glasgow. His research is concerned with the study of semiconductor lasers, LEDs, and photonic-integrated circuits for applications in energy efficient lighting and visible light communications. He is an associate editor of Optics Express and Journal of Nanophotonics. He is a fellow of OSA, SPIE, and IOP. 